\title{
DIFFUSION D'UNE ONDE PAR UN COIN
}

\author{
PATRICK GÉRARD ET GILLES LEBEAU
}

\section{SOMMAIRE}

1. Introduction et énoncé du résultat

2. Réduction au bord

2.1. Estimations $L^{2}$ des traces

2.2. Transformation FBI partielle en temps

2.3. Système vérifié par les dérivées normales

3. Réduction deux-microlocale

3.1. Opérateurs pseudo-différentiels dans le domaine complexe pour les problèmes aux limites

3.2. Régularité deux-microlocale pour le problème de Dirichlet

3.3. Changement d'inconnues et écriture du système

4. Microlocalisation du problème et développements asymptotiques

4.1. Transformation FBI en variable d'espace

4.2. Espaces de fonctions holomorphes

4.3. Développement des termes pseudo-différentiels

4.4. Développement des termes singuliers

4.5. Développement du second membre

4.6. Système microlocalisé

5. Inversion du symbole principal

6. Fin de la démonstration

Appendice A. Calcul pseudo-différentiel complexe à valeurs opérateurs dans une chaîne d'espaces de Banach

Appendice B. Développements asymptotiques

Appendice C. Choix de la chaîne d'espaces

Appendice D. Résolution d'un problème elliptique dans un secteur angulaire

Received by the editors December 24, 1991.

1991 Mathematics Subject Classification. Primary 35L05, 35S15.

Key words and phrases. Equations d'ondes, dièdre, singularités. 


\section{INTRODUCTION ET ÉNONCÉ DU RÉSULTAT}

Soient $a: \mathbb{R} \rightarrow \mathbb{R}$ et $b: \mathbb{R} \rightarrow \mathbb{R}$ deux fonctions $C^{\infty}$, analytiques près de l'origine, telles que

$$
a(0)=b(0)=0, \quad a^{\prime}(0)>0>b^{\prime}(0), \quad a(x)>0>b(x) \quad \text { pour } x>0 .
$$

On considère, dans $\mathbb{R}_{t} \times \mathbb{R}_{x, y}^{2}$, l'ouvert

$$
\begin{aligned}
& \Omega=\mathbb{R}_{t} \times\left(\mathbb{R}^{2} \backslash F\right) \\
& F=\left\{(x, y) \in \mathbb{R}^{2}, x \geq 0, b(x) \leq y \leq a(x)\right\} .
\end{aligned}
$$

Soit par ailleurs $u_{i} \in H_{\text {loc }}^{1}\left(\mathbb{R} \times \mathbb{R}^{2}\right)$ une solution de $\square=\partial_{t}^{2}-\Delta_{x, y}$ dans $\mathbb{R} \times \mathbb{R}^{2}$, conormale analytique par rapport à une surface $\Sigma_{i}$ de $\mathbb{R} \times \mathbb{R}^{2}$ telle que

$$
\Sigma_{i} \cap\{t<0\} \subset \Omega, \quad \Sigma_{i} \cap\{t=0\} \cap \partial \Omega=\{t=x=y=0\} .
$$

On suppose que $u_{i}$ est supportée du côté de $\Sigma_{i}$ ne rencontrant pas $\mathbb{R}_{t} \times F$ (voir la Figure 1).

Soit $u \in H_{\text {loc }}^{1}(\bar{\Omega})$ telle que

$$
\square u=0,\left.\quad u\right|_{\partial \Omega}=0,\left.\quad u\right|_{t<0}=\left.u_{i}\right|_{t<0} .
$$

On se propose de décrire les singularités de $u$ pour $t>0$ assez petit, en dehors des rayons diffractés tangentiellement aux demi-courbes $y=a(x), y=$ $b(x), x>0$.

Dans le cas où le coin est droit (i.e., $a$ et $b$ sont des fonctions linéaires), on peut calculer $u$ en passant en coordonnées polaires de centre $x=y=0$, et en utilisant les fonctions de Bessel. Ce calcul a été réalisé pour la première fois par Macdonald [M], puis précisé par Garnir [Ga], Varenne [V], Cheeger-Taylor $[C T]$, enfin Rouleux [R] dans le cadre analytique. Dans le cas général, Uchida $[U]$ a récemment obtenu des estimations géométriques a priori sur la diffraction des singularités analytiques.

On va ici donner un résultat quantitatif, c'est-à-dire conduisant à un calcul explicite de l'amplitude de diffusion. Avant d'énoncer ce résultat, nous devons

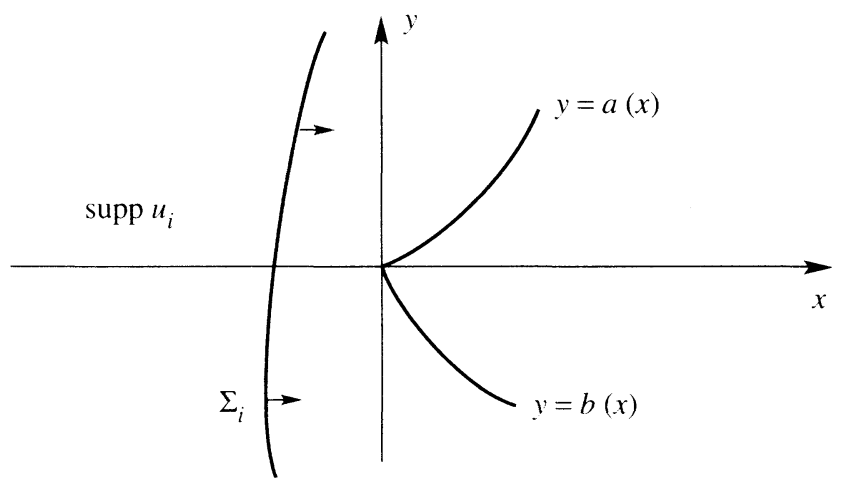

Figure 1. Situation à $t<0$. 
introduire quelques notations supplémentaires. On pose

$$
\left.a^{\prime}(0)=a=\operatorname{tg} \gamma_{+}, \quad b^{\prime}(0)=b=\operatorname{tg} \gamma_{-}, \quad \pm \gamma_{ \pm} \in\right] 0, \frac{\pi}{2}[.
$$

Dans un voisinage de $t=x=y=0$, on peut écrire

$$
u_{i}(t, x, y) \equiv \frac{1}{2 \pi} \int_{-\infty}^{\infty} e^{i \tau\left(t-\theta_{i}(x, y)\right)} \sigma_{i}(x, y, \tau) d \tau
$$

modulo une fonction analytique, où $\theta_{i}$ est une solution analytique réelle de l'équation eikonale

$$
\left(\frac{\partial \theta_{i}}{\partial x}\right)^{2}+\left(\frac{\partial \theta_{i}}{\partial y}\right)^{2}=1
$$

vérifiant de plus

$$
\theta_{i}(0,0)=0, \quad \frac{\partial \theta_{i}}{\partial x}(0,0)=1
$$

et où $\sigma_{i}$ est un symbole analytique se prolongeant en une fonction holomorphe dans $\operatorname{Im} \tau<0$, et vérifiant

$$
\sup _{s \geq 0} \int_{-\infty}^{\infty}\left(1+|\tau|^{2}\right)\left|\sigma_{i}(x, y, \tau-i s)\right|^{2} d \tau<+\infty .
$$

Notons par ailleurs

$\widetilde{\Delta}_{+}=\{(t, x, y), y=a(x)\}, \quad \tilde{\Delta}_{-}=\{(t, x, y), y=b(x)\}, \quad \Delta_{ \pm}=\widetilde{\Delta}_{ \pm} \cap\{x>0\}$, et désignons par $\delta_{ \pm}$les distributions en $(x, y)$ définies par

$$
\left\langle\delta_{+}, \varphi\right\rangle=\int_{0}^{\infty} \varphi(x, a(x)) d x, \quad\left\langle\delta_{-}, \varphi\right\rangle=\int_{0}^{\infty} \varphi(x, b(x)) d x .
$$

Enfin, on posera, lorsque les traces ont un sens,

$$
\begin{aligned}
& \partial_{+} f(x)=\left.\left(\partial_{y}-a^{\prime}(x) \partial_{x}\right) f\right|_{y-a(x)=0^{+}}, \\
& \partial_{-} f(x)=\left.\left(b^{\prime}(x) \partial_{x}-\partial_{y}\right) f\right|_{y-b(x)=0^{-}} .
\end{aligned}
$$

Soient $u_{ \pm}$les solutions de $\square u_{ \pm}=0,\left.u_{ \pm}\right|_{\tilde{\Delta}_{ \pm}}=0$, obtenues par réflexion de $u_{i}$ sur $\widetilde{\Delta}_{ \pm}$. Ces fonctions sont décrites explicitement par des différences d'intégrales de type (1.6) (voir la $\S 3.3$ pour un rappel de ces constructions standards). On note alors $u_{e}$ la solution dans $\mathbb{R} \times \mathbb{R}^{2}$ du problème

$$
\left\{\begin{array}{l}
\square u_{e}=-1_{x>0}\left(\partial_{+} u_{+} \delta_{+}+\partial_{-} u_{-} \delta_{-}\right), \\
\left.u_{e}\right|_{t<0}=\left.u_{i}\right|_{t<0} .
\end{array}\right.
$$

La fonction $u_{e}$ est dans $H_{\text {loc }}^{1}(\bar{\Omega})$, et est décrite explicitement par des intégrales de phase. En notant (voir la Figure 2, p. 344) $\Sigma_{r}$ la réunion des deux surfaces $\Sigma_{r, \pm}$ obtenues par réflexion de $\Sigma_{i}$ sur $\Delta_{ \pm}$, et un posant $\Sigma_{d}=\{(t, x, y), t=$ $\left.\sqrt{x^{2}+y^{2}}\right\}$, on peut en particulier montrer que

$$
\begin{aligned}
& u_{e} \text { est conormale analytique sur la partie lisse de } \Sigma_{i} \cup \Sigma_{r} \cup \Sigma_{d}, \\
& u_{e} \in H^{3 / 2} \text { près de } \Sigma_{d} \backslash\left(\Sigma_{i} \cup \Sigma_{r}\right), t>0
\end{aligned}
$$




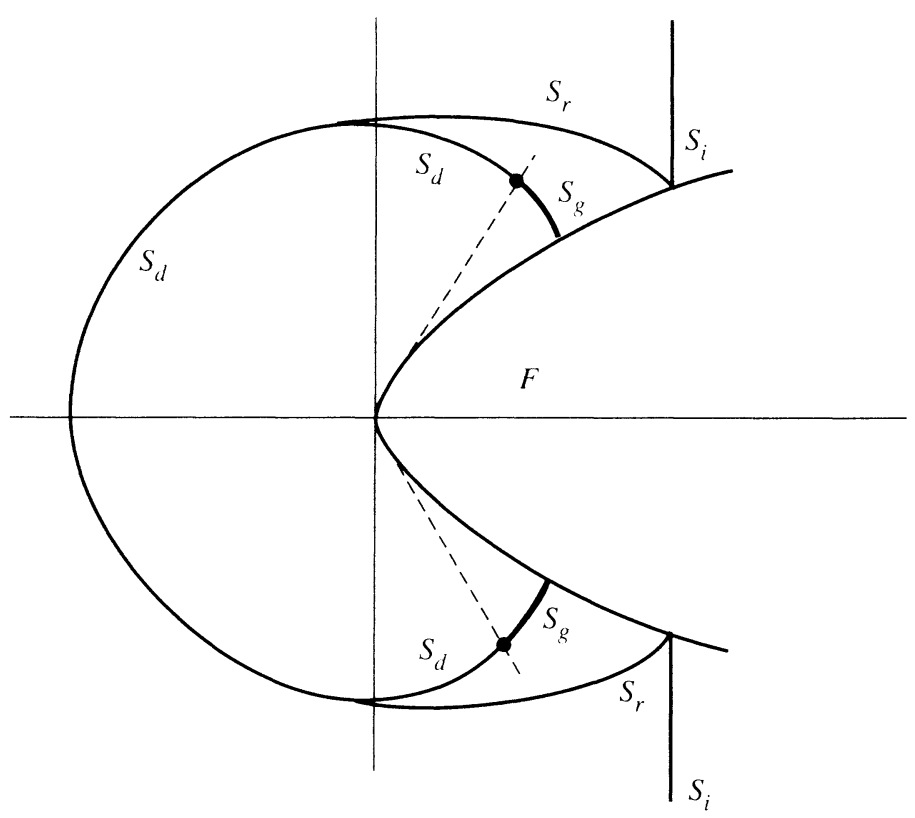

FIGURE 2. Situation à $t>0$ petit.

et calculer les symboles correspondants. Le but de ce travail est de décrire la différence $u-u_{e}$. On ne pourra en fait la décrire que dans

(1.14) $\Omega^{\varepsilon}=\mathbb{R}_{t} \times \mathbb{R}_{x, y}^{2} \backslash\left(F \cup\left\{(x, y), x \geq 0, \operatorname{tg}\left(\gamma_{-}-\varepsilon\right) x \leq y \leq \operatorname{tg}\left(\gamma_{+}+\varepsilon\right) x\right\}\right)$

où $\varepsilon>0$ est arbitrairement petit.

Théorème 1.1. Pour tout $\varepsilon>0$, il existe $\varepsilon_{1}>0$ tel que, pour $(t, x, y) \in \Omega^{\varepsilon}$, $0<t<\varepsilon_{1}$,

$$
u(t, x, y)=u_{e}(t, x, y)+u_{d}(t, x, y),
$$

ò̀ $u_{d} \in H_{\mathrm{loc}}^{3 / 2}\left(\bar{\Omega}^{\varepsilon}\right)$ est conormale analytique par rapport $\grave{a} \Sigma_{d}$, c'est-à-dire

$$
u_{d}(t, x, y)=\int_{-\infty}^{+\infty} e^{i \tau\left(t-\sqrt{\left.x^{2}+y^{2}\right)}\right.} \sigma_{d}(x, y, \tau) \frac{d \tau}{2 \pi} .
$$

modulo une fonction analytique, $\sigma_{d}$ étant un symbole analytique, holomorphe dans $\operatorname{Im} \tau<0$, vérifiant

$$
\sup _{s \geq 0} \int_{-\infty}^{\infty}\left(1+|\tau|^{2}\right)^{3 / 2}\left|\sigma_{d}(x, y, \tau-i s)\right|^{2} d \tau<+\infty .
$$

De plus, on a

$$
\sigma_{d}(x, y, \tau)=\sigma_{d}^{0}(x, y, \tau)+r^{0}(x, y, \tau)
$$

où $r$ est un symbole analytique vérifiant

$$
\sup _{s \geq 0} \int_{-\infty}^{\infty}\left(1+|\tau|^{2}\right)^{5 / 2}\left|r^{0}(x, y, \tau-i s)\right|^{2} d \tau<+\infty .
$$


Le terme principal $\sigma_{d}^{0}$ pouvant s'écrire

$$
\begin{aligned}
\sigma_{d}^{0}(x, y, \tau)=\left(\tau \sqrt{x^{2}+y^{2}}\right)^{-1 / 2} \sigma_{i}(0,0, \tau)\left(K_{+}\left(\cos \left(\theta-\gamma_{+}\right)\right)\right. \\
\left.+K_{-}\left(\cos \left(\theta-\gamma_{-}\right)\right)\right)
\end{aligned}
$$

où $\theta \in] \gamma_{+}+\varepsilon, 2 \pi+\gamma_{-}-\varepsilon\left[\right.$ est défini par $\cos \theta=x / \sqrt{x^{2}+y^{2}}, \sin \theta=$ $y / \sqrt{x^{2}+y^{2}}$, et où $K_{ \pm}$sont des fonctions analytiques ne dépendant que de $\gamma_{+}$ et $\gamma_{-}$.

On va décrire dans les sections qui suivent une procédure explicite de calcul des fonctions $K_{ \pm}$, qui permet également de déterminer tous les termes du développement asymptotique de $\sigma_{d}$. Auparavant, on notera que, compte tenu de (1.13), le théorème ci-dessus entraîne le:

Corollaire 1.1. Pour tout $\varepsilon>0$, il existe $\varepsilon_{1}>0$ tel que la fonction $u$ soit conormale analytique sur la partie lisse de $\Sigma_{d} \cup \Sigma_{r} \cup \Sigma_{i}$ dans $\Omega^{\varepsilon} \cap\left\{0<t<\varepsilon_{1}\right\}$, et $u \in H^{3 / 2}$ près de $\Sigma_{d} \backslash\left(\Sigma_{r} \cup \Sigma_{i}\right)$ dans $\Omega^{\varepsilon} \cap\left\{0<t<\varepsilon_{1}\right\}$.

On déduira aussi des calculs effectués le Théorème 1.2 suivant de propagation des singularités. Soit $L$ l'arête $L=\{(t ; x=y=0)\}$ et

$$
T_{b}^{*} \Omega=T^{*} \Omega \cup T^{*} \Delta_{+} \cup T^{*} \Delta_{-} \cup T^{*} L .
$$

La topologie sur $T_{b}^{*} \Omega$ est définie par la projection naturelle $p: T^{*} \bar{\Omega} \rightarrow T_{b}^{*} \Omega$. Par définition un rayon est une application continue

$$
\rho: I \rightarrow p((\operatorname{car} \square) \cap(\tau, \xi, \eta) \neq 0),
$$

où $I$ est un intervalle ouvert de $\mathbb{R}$, telle que $\rho$ soit un rayon au sens usuel près

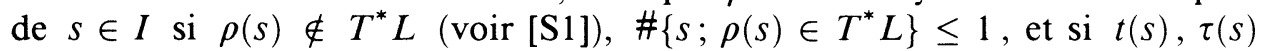
sont les coordonnées $(t, \tau)$ de $\rho(s)$, on a $\tau(s)=\tau_{0} \neq 0$ et $t(s)=t-2 \tau_{0} s$.

Soit $S$ le sous-ensemble de $T^{*} \Omega$ défini par

$$
S=\left\{q \in T^{*} \Omega ; \text { il existe un rayon } \rho, q \in \rho \text {, et }(\rho \cap t<0) \subset T_{\Sigma_{i}}^{*}\right\} .
$$

On peut écrire $S$ comme la réunion disjointe

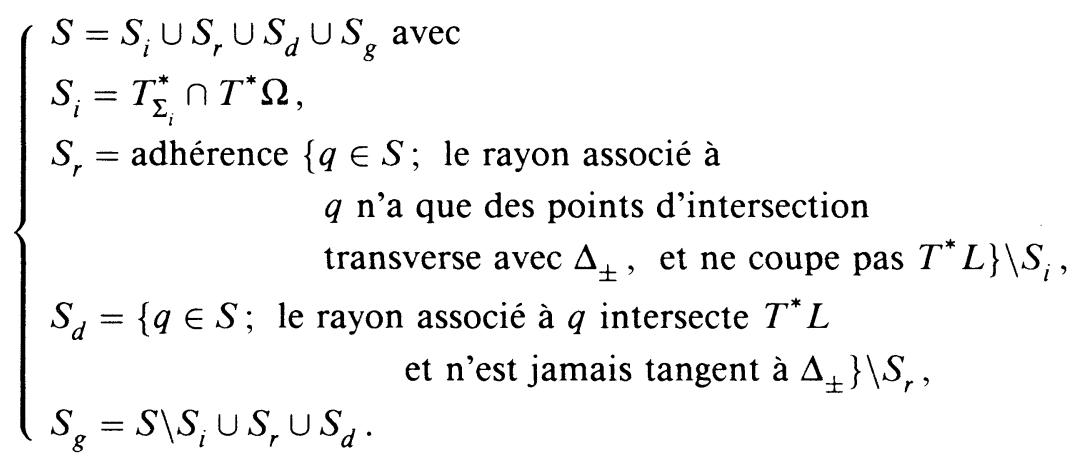

(On a dessiné sur la Figure 2 la projection sur la base $(x, y)$ de $S \cap\left(t=t_{0}>0\right)$ dans le cas $a$ concave, $b$ convexe, $u_{i}$ onde plane.)

On a alors: 
Théorème 1.2. Le front d'onde analytique de $u$ dans $T^{*} \Omega$ est contenu dans $S$ près de $x=y=t=0$.

Ces résultats sont en accord avec la théorie géométrique de la diffraction de Keller [Ke] (voir aussi Molinet [Mo] pour une introduction à cette théorie et une bibliographie détaillée). En particulier, la relation (1.20) est une illustration de "l'approximation du plan tangent", qui affirme que la partie principale de l'onde diffractée est la même que si le coin était droit.

La clé du théorème précédent est un théorème de régularité 2 -microlocale pour un problème aux limites dans un demi-espace, basé sur la stratégie de déformation microhyperbolique introduite par Kashiwara-Kawaï [KK] et Sjöstrand $[S 2,3]$. Ce résultat permet de faire une analyse asymptotique de l'opérateur de bord associé au problème (1.4), et de se ramener ainsi à l'inversion d'un système d'équations intégrales relié au même problème pour un coin droit. Ce dernier système est luimême résolu par une méthode de déformation dans le domaine complexe, qui permet de se ramener à la résolution d'un problème elliptique.

Cet article s'organise de la façon suivante. Dans la $\S 2$, on prouve que les dérivées normales $v_{ \pm}$de $u$ sur $\Delta_{ \pm}$sont $L^{2}$. On introduit ensuite une transformation de Fourier-Bros-Iagolnitzer (FBI) partielle $T_{0}$ dans la variable $t$, qui permet déjà de microlocaliser le problème près de $t=0$ et (par exemple) $\tau=1$. On écrit enfin le système vérifié par $v_{ \pm}$, que le caractère singulier des opérateurs de bord oblige à interpréter dans un espace $E_{1}$ de distributions supportées dans $t \geq 0$, qui contient strictement $L^{2}$.

La $\S 3$ est consacrée au théorème de régularité deux-microlocale évoqué plus haut. Après avoir développé un calcul symbolique pseudo-différentiel dans le domaine complexe pour les problèmes aux limites (qui ne differe pas essentiellement de celui de Sjöstrand [S1, 2, 3]), on utilise cette technique pour démontrer un résultat de régularité pour les solutions d'un problème de Dirichlet dans un demi-espace, qui sont "sortantes" relativement à une sous-variété involutive de codimension 1 du cotangent au bord. Le résultat s'exprime en termes du second microsupport à croissance le long de $V$, tel qu'il a été introduit dans [L1]. La fin de la $\S 3$ applique ce résultat au problème étudié: on montre que, si l'on enlève à $v_{ \pm}$les contributions des ondes réfléchies $u_{ \pm}$, on obtient deux nouvelles inconnues $f_{ \pm}$qui sont deux-microlocalement régulières par rapport à la variété involutive $V=\{x=0\}$, sauf près des directions qui émettent tangentiellement aux demi-courbes $\Delta_{ \pm}$.

La $\S 4$ a pour objet la microlocalisation en $x$ du système portant sur $f_{ \pm}$. On introduit d'abord une transformation FBI $T_{1}$ en $x$; le résultat de la section précédente se traduit en termes de croissance modérée, par rapport au grand paramètre $\lambda$, de la fonction holomorphe $T_{0} \otimes T_{1}\left(f_{ \pm}\right)(w, z, \lambda)$ dans un domaine $W \times \omega$ de $\mathbb{C}_{w} \times \mathbb{C}_{z}$. Le reste de la section consiste à tirer parti de cette estimation pour transformer le système sur $f_{ \pm}$en un système sur $T_{0} \otimes T_{1}\left(f_{ \pm}\right)$, que l'on ramène à un système pseudo-différentiel en $w$ à valeurs opérateurs dans une chaîne d'espaces de fonctions holomorphes en $z$ (au sens d'Ovsjannikov; voir $[\mathrm{O}],[\mathrm{N}],[\mathrm{BG}])$. Pour inverser ce système, on est alors ramené à prouver l'inversibilité de son symbole principal dans la chaîne d'espaces en question. 
Dans la $\S 5$, on prouve l'inversibilité du symbole principal en faisant effectuer au contour de cet opérateur intégral une légère rotation dans le domaine complexe. On constate alors que le système "déformé" est équivalent à un problème elliptique dans un secteur angulaire du plan des $(x, y)$, dont on montre qu'il est bien posé dans l'appendice D.

Enfin, la $\S 6$ utilise le calcul pseudo-différentiel à valeurs opérateurs présenté en Appendice A pour en déduire $T_{0} \otimes T_{1}\left(f_{ \pm}\right)$. L'onde diffractée $u_{d}$ est alors la solution, nulle dans $t<0$, de l'équation $\square u_{d}=-\left(f_{+} \delta_{+}+f_{-} \delta_{-}\right)$.

Nous terminons cette introduction en rappelant l'inégalité de Hardy suivante, que nous utiliserons à de nombreuses reprises au cours de la démonstration:

Proposition 1.1. L'opérateur de noyau $1_{x \geq 0, y \geq 0}(1 /(x+y))$ est borné sur $L^{2}\left(\mathbb{R}_{+}\right)$. Démonstration. Désignons par $A$ l'opérateur en question, et par $A_{1}$ l'opérateur de noyau

$$
K_{1}(x, y)=1_{x \geq y \geq 0} \frac{1}{x+y} .
$$

Alors on a $A=A_{1}+A_{1}^{*}$, et il suffit de montrer que $A_{1}$ est borné sur $L^{2}\left(\mathbb{R}_{+}\right)$. Or

$$
\left|A_{1} f(x)\right|=\left|\int_{0}^{x} \frac{f(y)}{x+y} d y\right| \leq \frac{1}{x} \int_{0}^{x}|f(y)| d y,
$$

et il suffit de montrer que l'opérateur $A_{2}$ défini par

$$
A_{2} f(x)=\frac{1}{x} \int_{0}^{x} f(y) d y=\int_{0}^{1} f(t x) d t
$$

est borné sur $L^{2}\left(\mathbb{R}_{+}\right)$. Or, si $g \in L^{2}\left(\mathbb{R}_{+}\right)$,

$$
\begin{gathered}
\left(A_{2} f, g\right)=\int_{0}^{1}\left(\int_{0}^{\infty} f(t x) \overline{g(x)} d x\right) d t \leq\|g\| M, \\
M=\int_{0}^{1}\left(\int_{0}^{\infty}|f(t x)|^{2} d x\right)^{1 / 2} d t=\|f\| \int_{0}^{1} \frac{d t}{\sqrt{t}}=2\|f\|,
\end{gathered}
$$

ce qu'il fallait démontrer.

\section{RÉDUCTION AU BORD}

2.1. Estimations $L^{2}$ des traces. Si $u$ est la solution introduite à la section précédente, on peut définir

$$
v_{ \pm}=\left.\left(\partial_{ \pm} u\right)\right|_{x>0} \in \mathscr{D}^{\prime}\left(\Delta_{ \pm}\right) .
$$

Par hypothèse, $\operatorname{supp}\left(v_{ \pm}\right) \subset\{t \geq 0\}$, et, par vitesse finie de propagation, on a $\operatorname{supp}\left(v_{ \pm}\right) \subset\{(t, x), 0 \leq x \leq c t\}$. On désigne par $\underline{u}$ le prolongement de $u$ par 0 en dehors de $\Omega$. On a alors $\underline{u} \in H_{\text {loc }}^{1}\left(\mathbb{R}_{t} \times \mathbb{R}_{x, y}^{2}\right)$. (Voir Grisvard [G].)

Proposition 2.1. Pour tout $T>0$ assez petit, $v_{ \pm} \in L^{2}\left([0, T] \times \mathbb{R}_{+}\right)$, et on a lidentité

$$
\square \underline{u}=-v_{+} \delta_{+}-v_{-} \delta_{-} .
$$


Démonstration. Soit $\chi \in C_{0}^{\infty}(]-T, T[\times B(0, r))$ et soit $\rho_{\varepsilon}$ la composée de $\chi$ avec une régularisation en temps. Alors $u_{\varepsilon}=\rho_{\varepsilon} u$ est bornée dans $H^{1}$ et vérifie

$$
\left\{\begin{array}{l}
\Delta u_{\varepsilon}=\left[\Delta, \rho_{\varepsilon}\right] u+\partial_{t}^{2} \rho_{\varepsilon} u \in L^{2}, \\
\left.u_{\varepsilon}\right|_{\partial \Omega}=0,
\end{array}\right.
$$

ce qui rend licites des formules d'intégration par parties sur $\Omega$ ([G]). Il s'agit de montrer que $\left\|\partial u_{\varepsilon} / \partial n\right\|_{L^{2}\left(\Delta_{ \pm}\right)}$est bornée en $\varepsilon$. Pour cela, on choisit un champ réel $X$ sur $\mathbb{R}_{x, y}^{2}$ tel que $X \cdot n \geq c_{0}>0$ sur $\partial \Omega, n$ étant la normale sortante, définie sur $\Delta_{+} \cup \Delta_{-}$, donc presque partout sur $\partial \Omega$. Le calcul ci-dessous est alors essentiellement classique; on $y$ désignera par $0(1)$ toute quantité bornée uniformément en $\varepsilon$, et on fera un usage constant du fait que $\left\|u_{\varepsilon}\right\|_{H^{1}(\Omega)}=0(1)$. On a

$$
\left(\left(\partial_{t}^{2}-\Delta\right) u_{\varepsilon}, X u_{\varepsilon}\right)=\left(\left[\partial_{t}^{2}-\Delta, \rho_{\varepsilon}\right] u, X u_{\varepsilon}\right)=0(1)
$$

car $\rho_{\varepsilon}$ est une famille bornée d'opérateurs d'ordre 0 . Par ailleurs,

$$
\operatorname{Re}\left(\left(\partial_{t}^{2}-\Delta\right) u_{\varepsilon}, X u_{\varepsilon}\right)=-\operatorname{Re}\left(\partial_{t} u_{\varepsilon}, X \partial_{t} u_{\varepsilon}\right)-\operatorname{Re}\left(\Delta u_{\varepsilon}, X u_{\varepsilon}\right)
$$

Comme $\left.\partial_{t} u_{\varepsilon}\right|_{\partial \Omega}=0$ et que $X$ est un champ réel,

$$
\operatorname{Re}\left(\partial_{t} u_{\varepsilon}, X \partial_{t} u_{\varepsilon}\right)=0(1) \text {. }
$$

D'autre part

$$
\begin{aligned}
0= & \operatorname{Re}\left(\Delta u_{\varepsilon}, X u_{\varepsilon}\right)+\operatorname{Re}\left(\nabla u_{\varepsilon}, \nabla X u_{\varepsilon}\right)-\operatorname{Re}\left(\frac{\partial u_{\varepsilon}}{\partial n}, X u_{\varepsilon}\right)_{\partial \Omega} \\
= & \operatorname{Re}\left(\Delta u_{\varepsilon}, X u_{\varepsilon}\right)+0(1)+\operatorname{Re}\left(\nabla u_{\varepsilon}, X \nabla u_{\varepsilon}\right) \\
& -\operatorname{Re}\left(\frac{\partial u_{\varepsilon}}{\partial n}, X u_{\varepsilon}\right)_{\partial \Omega} .
\end{aligned}
$$

Or, $X$ étant un champ réel,

$$
\operatorname{Re}\left(\nabla u_{\varepsilon}, X \nabla u_{\varepsilon}\right)=0(1)+\frac{1}{2}\left(X \cdot n \nabla u_{\varepsilon}, \nabla u_{\varepsilon}\right)_{\partial \Omega} .
$$

En injectant (2.4), (2.5), et (2.6) dans (2.3), et en comparant avec (2.2), il vient

$$
\frac{1}{2}\left(X \cdot n \nabla u_{\varepsilon}, \nabla u_{\varepsilon}\right)_{\partial \Omega}-\operatorname{Re}\left(\frac{\partial u_{\varepsilon}}{\partial n}, X u_{\varepsilon}\right)_{\partial \Omega}=0(1),
$$

ce qui, puisque $\left.u_{\varepsilon}\right|_{\partial \Omega}=0$, s'écrit

$$
-\frac{1}{2}\left\|\sqrt{X \cdot n} \frac{\partial u_{\varepsilon}}{\partial n}\right\|_{L^{2}(\partial \Omega)}=0(1)
$$

ce qu'il fallait démontrer.

Passons à la deuxième assertion de la Proposition 1.1.

Puisque $\left.u\right|_{\Delta_{ \pm}}=0$, la formule des sauts usuelle entraîne que la distribution $\square \underline{u}+v_{+} \delta_{+} v_{-} \delta_{-}$est supportée par $x=y=0$. Or, d'après les informations sur $v_{ \pm}$et sur $\underline{u}$, c'est un élément de $H_{\text {loc }}^{-1}\left(\mathbb{R}^{3}\right)$. Elle est donc nulle. 
Nous allons déduire de la formule (2.1) un système d'équations sur $v_{ \pm}$. Pour cela, on désigne par $\square^{-1}$ la solution fondamentale de $\square$ à support dans $t \geq 0$. Puisque $\left.\underline{u}\right|_{t<0}=\left.u_{i}\right|_{t<0}$, la formule (2.1) s'écrit

$$
\underline{u}=u_{i}-\square^{-1}\left(v_{+} \delta_{+}+v_{-} \delta_{-}\right) .
$$

2.2. Transformation FBI partielle en $t$. On pose $\mathbb{C}^{-}=\{\tau \in \mathbb{C}, \operatorname{Im} \tau<0\}$. Pour $u(t, x)$ distribution tempérée en $(t, x) \in \mathbb{R}^{1+d}$ on note $\hat{u}(\tau, x)$ la transformée de Fourier partielle en $t$ et pour $\lambda \geq 1, \hat{u}_{\lambda}(\tau, x)=\hat{u}(\lambda \tau, x)$.

Définition 2.1. Soit $e \in \mathbb{R}_{+}$. On note $E_{e}$ l'ensemble des distributions tempérées $u(t, x)$, à support dans $t \geq 0$ telles que

i) $\forall \tau \in \mathbb{C}^{-}, \hat{u}(\tau, \cdot) \in L^{2}\left(\mathbb{R}^{d}\right)$;

ii) $\exists A, B, \forall \tau \in \mathbb{C}^{-},\|\hat{u}(\tau, \cdot)\|_{L^{2}} \leq \frac{A(1+|\tau|)^{B}}{|\operatorname{Im} \tau|^{e}}$.

Pour $w \in \mathbb{C}, \lambda \geq 1$, et $u \in E_{e}$ on pose

$$
T_{0} u(w, x, \lambda)=\sqrt{\frac{\lambda}{2 \pi}} \int_{\operatorname{Im} \tau=-a} e^{i \lambda \tau w-\lambda \tau^{2} / 2} \hat{u}_{\lambda}(\tau, x) d \tau
$$

avec $a>0$, l'intégrale étant indépendante de $a$.

Pour $t \in \mathbb{R}, \tau \in \mathbb{C}$, on a

$$
e^{-\lambda \tau^{2} / 2} T_{0} u(t-i \tau, x, \lambda)=\sqrt{\frac{\lambda}{2 \pi}} \int_{\operatorname{Im} \sigma=-a} e^{i \lambda \sigma t-\lambda(\sigma-\tau)^{2} / 2} \hat{u}_{\lambda}(\sigma, x) d \sigma
$$

Il en résulte qu'il existe des constantes $A$ et $B$ telles qu'on ait

$$
\begin{gathered}
t+\operatorname{Im} \tau<0 \Rightarrow\left\|e^{-\lambda \tau^{2} / 2} T_{0} u(t-i \tau, \cdot, \lambda)\right\|_{L_{x}^{2}} \\
\leq e^{-\lambda t^{2} / 2-\lambda t \operatorname{Im} \tau} \frac{A(\lambda+|\tau|+|t|)^{B}}{|t+\operatorname{Im} \tau|^{e}} \\
t+\operatorname{Im} \tau>-1 \Rightarrow\left\|e^{-\lambda \tau^{2} / 2} T_{0} u(t-i \tau, \cdot, \lambda)\right\|_{L_{x}^{2}} \\
\leq e^{\lambda(\operatorname{Im} \tau)^{2} / 2} A(\lambda+|\operatorname{Re} \tau|)^{B} \\
\times(2+t+\operatorname{Im} \tau)^{e}
\end{gathered}
$$

En effet, (2.10) s'obtient en choisissant $a=-(t+\operatorname{Im} \tau)$ dans (2.9) et (2.11) en choisissant $a=1 / \lambda(2+t+\operatorname{Im} \tau)$.

Lemme 2.1. On a la formule d'inversion, pour $u \in E_{e}$,

$$
\hat{u}_{\lambda}(\tau, x)=\sqrt{\frac{\lambda}{2 \pi}} \int_{-\infty}^{+\infty} e^{-i \lambda \tau t} e^{-\lambda \tau^{2} / 2} T_{0} u(t-i \tau, x, \lambda) d t .
$$

Preuve. D'après (2.10), (2.11), et $\operatorname{Im} \tau<0$ l'intégrale de droite est normalement convergente dans $L_{x}^{2}$. Soit $\theta \in C_{0}^{\infty}(\mathbb{R}), \theta \equiv 1$ près de l'origine. Soit $I$ le 
membre de droite de (2.12). D'après (2.9) on a

$$
\begin{aligned}
I & =\lim _{\varepsilon \rightarrow O+} \frac{\lambda}{2 \pi} \int_{-\infty}^{+\infty} e^{-i \lambda \tau t} \theta(\varepsilon t) \int_{\operatorname{Im} \sigma=\operatorname{Im} \tau} e^{i \lambda \sigma t-\lambda(\sigma-\tau)^{2} / 2} \hat{u}_{\lambda}(\sigma, x) d \sigma d t \\
& =\lim _{\varepsilon \rightarrow O+} \int_{-\infty}^{+\infty} \frac{\lambda}{2 \pi \varepsilon} \hat{\theta}\left(\frac{\lambda u}{\varepsilon}\right) e^{-\lambda u^{2} / 2} \hat{u}_{\lambda}(\tau-u, x) d u
\end{aligned}
$$

et le lemme résulte de $\lim _{\varepsilon \rightarrow 0}(\lambda / 2 \pi \varepsilon) \hat{\theta}(\lambda u / \varepsilon)=\delta_{u=0}$ dans $\mathscr{S}_{u}^{\prime}$.

Définition 2.2. Pour $W$ ouvert de $\mathbb{C}$ et $\varphi \in C^{0}(W, \mathbb{R})$ on note $H_{\varphi}\left(W, L^{2}\right)$ l'espace de Sjöstrand des fonctions $f(w, x, \lambda)$ définies pour $w \in W, x \in \mathbb{R}^{d}$, $\lambda \geq 1$, holomorphes en $w, L^{2}$ en $x$, telles que

$$
\forall K \Subset W, \quad \exists A, B, \quad \sup _{w \in K}\left\|e^{-\lambda \varphi(w)} f(w, \cdot, \lambda)\right\|_{L_{x}^{2}} \leq A \lambda^{B} .
$$

On notera $\varphi_{0}=(\operatorname{Im} w)^{2} / 2, \tilde{\varphi}_{0}=(\operatorname{Im} w)^{2} / 2-\left[(\operatorname{Re} w)_{-}\right]^{2} / 2$ où $(\operatorname{Re} w)_{-}=0$ pour $\operatorname{Re} w \geq 0 ;(\operatorname{Re} w)_{-}=\operatorname{Re} w$ pour $(\operatorname{Re} w) \leq 0$. Il résulte de $(2.10)$ et (2.11) qu'on a, pour $u \in E_{e}$,

$$
T_{0} u \in H_{\tilde{\varphi}_{0}}\left(\mathbb{C}, L^{2}\right) .
$$

On choisit un réel $\kappa>0$ et on pose

$$
W=\{w,|\operatorname{Re} w|<\kappa,|1+\operatorname{Im} w|<\kappa\} .
$$

Lemme 2.2. Soit $u \in E_{e}$ avec support $(u) \subset t \geq t_{0}>\kappa$. On a

$$
T_{0} u \in H_{\varphi}\left(W, L^{2}\right), \quad \varphi=\varphi_{0}-\frac{1}{2}\left(t_{0}-\kappa\right)^{2} .
$$

Preuve. Soit $\psi \in C^{\infty}(\mathbb{R}), \psi(t) \equiv 1$ pour $t \geq t_{0}-\varepsilon, \psi(t) \equiv 0$ pour $t \leq t_{0}-2 \varepsilon$ où $\varepsilon>0$ est petit. On a alors $\psi u=u$ et

$$
T_{0} u(w, x, \lambda)=\left(\frac{\lambda}{2 \pi}\right)^{3 / 2} \int_{\operatorname{Im} \tau=-b} e^{i \lambda \tau w-\lambda \tau^{2} / 2} \hat{\psi}_{\lambda}(\tau-\sigma) \hat{u}_{\lambda}(\sigma, x) d \sigma .
$$

Or on a $\left|\hat{\psi}_{\lambda}(\tau)\right| \leq e^{\lambda \operatorname{Im} \tau\left(t_{0}-2 \varepsilon\right)} C_{N}(\lambda|\tau|)^{-N}$ et on conclut en choisissant $a$ près de 0 et $b=t_{0}-\kappa+\varepsilon$, avec $\varepsilon$ tel que $|\operatorname{Re} w| \leq \kappa-4 \varepsilon$ pour $w \in K \Subset W$.

Soit à présent $K_{\tau}$ une famille d'opérateurs bornés sur $L_{x}^{2}$, dépendant holomorphiquement de $\tau \in \mathbb{C}_{\text {_ }}$ telle que

$$
\exists A, B, e, \quad \forall \tau \in \mathbb{C}_{-}, \quad\left\|K_{\tau}\right\| \leq A \frac{(1+|\tau|)^{B}}{|\operatorname{Im} \tau|^{e}} .
$$

Alors $K_{\tau}$ définit un opérateur $K$ de $E_{e^{\prime}}$ dans $E_{e+e^{\prime}}$ par

$$
\widehat{K u}(\tau, x)=K_{\tau} \hat{u}(\tau, x) \text {. }
$$

Proposition 2.2. Pour $u \in E_{e^{\prime}}$ on $a$

$$
\begin{aligned}
T_{0} K u & -\frac{\lambda}{2 \pi} \int_{t_{-}}^{t_{+}} d t \\
& \times \int_{\tau_{-}}^{\tau_{+}} d \tau e^{i \lambda \tau(w-t)-\lambda \tau^{2}} K_{\lambda \tau} T_{0} u(t-i \tau, \cdot, \lambda) \in H_{\varphi}\left(W, L^{2}\right)
\end{aligned}
$$


avec $\varphi=\varphi_{0}-\kappa^{2} / 4, t_{ \pm}= \pm 2 \kappa, \tau_{ \pm}=1 \pm 2 \kappa-i / \lambda$.

Preuve. D'après $(2.8)$ et (2.12), on a

$$
\begin{aligned}
T_{0} K u(w, x, \lambda)= & \frac{\lambda}{2 \pi} \int_{\operatorname{Im} \tau=-a} d \tau \\
& \times \int_{-\infty}^{+\infty} d t e^{i \lambda \tau(w-t)-\lambda \tau^{2} / 2} K_{\lambda \tau} e^{-\lambda \tau^{2} / 2} T_{0} u(t-i \tau, \cdot, \lambda) ;
\end{aligned}
$$

l'intégrale étant normalement convergente dans $L^{2}$ d'après (2.10), (2.11), et (2.17). Choisissons $a=1 / \lambda$; d'après (2.10) et (2.11) les intégrales dans (2.20) calculées dans $|\operatorname{Re} \tau-1| \geq 2 \kappa$ ou $t \leq-2 \kappa$ appartiennent à $H_{\varphi_{0}-\kappa^{2} / 4}\left(W, L^{2}\right)$. Pour $t \geq 2 \kappa$, il suffit alors de choisir comme contour de $\tau_{-}$à $\tau_{+}$

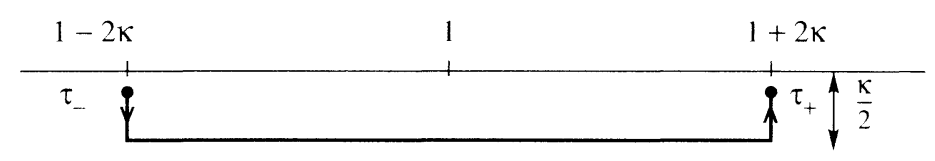

pour vérifier que la contribution à (2.20) de $\int_{2 \kappa}^{\infty} \int_{\tau_{-}}^{\tau_{+}}$appartient à $H_{\varphi_{0}-\kappa^{2} / 4}\left(W, L^{2}\right)$.

2.3. Système vérifié par les dérivées normales. Rappelons que $v_{+}, v_{-}$sont $L_{\text {loc }}^{2}$, à support dans $t \geq 0$ et $0 \leq x \leq c t$. Fixons $t_{0}>0$ petit et choisissons $\varphi_{1}(t), \varphi_{2}(t) \in C_{0}^{\infty}, \varphi_{1}(t) \equiv 1$ près de $t \leq t_{0}, \varphi_{1}(t) \equiv 0$ pour $t \geq 2 t_{0}, \varphi_{2}(t) \equiv 1$ pour $t \leq t_{0} / 2, \varphi_{2}(t) \equiv 0$ pour $t \geq t_{0}$ ainsi que $\psi(x) \in C_{0}^{\infty}$ avec $\psi(x) \equiv 1$ près de $|x| \leq 3 c t_{0}$. D'après (2.7), on a l'identité

$$
\varphi_{2} \underline{u}=\varphi_{2} u_{i}-\varphi_{2} \square^{-1}\left(\varphi_{1} v_{+} \delta_{+}+\varphi_{1} v_{-} \delta_{-}\right) .
$$

Pour $\tau \in \mathbb{C}^{-}$, on définit les opérateurs $K_{\tau}^{1,+}, K_{\tau}^{2,+}$ sur $L^{2}(\mathbb{R})$ par

$$
\begin{aligned}
& K_{\tau}^{1,+}(f)=\psi\left[\partial_{+}\left(-\tau^{2}-\Delta\right)^{-1}\left(1_{x \geq 0} f \psi \delta_{+}\right)\right], \\
& K_{\tau}^{2,+}(f)=\psi\left[\partial_{-}\left(-\tau^{2}-\Delta\right)^{-1}\left(1_{x \geq 0} f \psi \delta_{+}\right)\right],
\end{aligned}
$$

où $\left(-\tau^{2}-\Delta\right)^{-1}$ est l'o.p.d. de symbole $\left(\xi^{2}+\eta^{2}-\tau^{2}\right)$ sur $\mathbb{R}^{2}$. On remarquera que $\left(-\tau^{2}-\Delta\right)^{-1}\left(1_{x \geq 0} f \psi \delta_{+}\right)$est solution de l'opérateur $-\tau^{2}-\Delta$ sur $\mathbb{R}^{2} \backslash F$, que $\widetilde{\Delta}_{ \pm}$sont non caractéristiques pour $\Delta$, de sorte que $K_{\tau}^{i_{+}+}$sont bien définis. On pose

$$
\begin{aligned}
\mu\left(x, x^{\prime}\right) & =\frac{a(x)-a\left(x^{\prime}\right)}{x-x^{\prime}} ; \quad \nu\left(x, x^{\prime}\right)=\frac{b(x)-b\left(x^{\prime}\right)}{x-x^{\prime}} . \\
\xi_{+} & =\left(\left(1+\mu^{2}\right) \tau^{2}-\xi^{2}\right)^{1 / 2}, \quad \operatorname{Im} \xi_{+}>0 \\
\xi_{-} & =\left(\left(1+\nu^{2}\right) \tau^{2}-\xi^{2}\right)^{1 / 2}, \quad \operatorname{Im} \xi_{-}>0 .
\end{aligned}
$$


Par un calcul standard dans la théorie des problèmes aux limites elliptiques on trouve

$$
\begin{gathered}
\left\{\begin{array}{c}
K_{\tau}^{1,+}(f)(x)=-\frac{1}{4 \pi} \int e^{i\left(x-x^{\prime}\right) \xi} \psi(x) \sigma^{1}\left(x, x^{\prime}, \tau, \xi\right) \\
\times 1_{x^{\prime} \geq 0} f\left(x^{\prime}\right) \psi\left(x^{\prime}\right) d \xi \\
\sigma^{1}\left(x, x^{\prime}, \tau, \xi\right)=1+\frac{\mu-a^{\prime}(x)}{1+\mu^{2}}\left(\frac{\xi}{\xi_{+}}-\mu\right) .
\end{array}\right. \\
\left\{\begin{array}{l}
K_{\tau}^{2,+}(f)(x)=\lim _{\substack{\mathscr{D}^{\prime} \\
0^{+}}}-\frac{1}{4 \pi} \int e^{i\left(x-x^{\prime}\right) \xi} e^{i \phi_{\varepsilon}\left(x, x^{\prime}, \tau, \xi\right)} \psi(x) \\
\sigma^{2}\left(x, x^{\prime}, \tau, \xi\right)=\frac{1}{1+\nu^{2}}\left[\frac{\xi}{\xi}\left(b_{-}^{\prime}(x)-\nu\right)+1+\nu b^{\prime}(x)\right] \\
\phi_{\varepsilon}\left(x, x^{\prime}, \tau, \xi\right)=\left[b\left(x^{\prime}\right)-a\left(x^{\prime}\right)-\varepsilon\right] \frac{\nu \xi-\xi_{-}}{1+\nu^{2}}
\end{array}\right.
\end{gathered}
$$

Lemme 2.3. Les opérateurs $K_{\tau}^{i,+}$ sont bornés sur $L^{2}$ et vérifient les estimations de (2.17) avec $e=1$.

Preuve. Dans chacun des cas, on coupe l'intégrale en $\xi$ en deux morceaux, I: $|\xi| \leq C_{0}(1+|\tau|)$, II: $|\xi| \geq C_{0}(1+|\tau|)$, où $C_{0}$ est grand.

Pour traiter I, on remarque qu'on a

$$
\begin{aligned}
|\xi| \leq C_{0}(1+|\tau|) & \Rightarrow\left|\frac{\xi}{\xi_{+}}\right|+\left|\frac{\xi}{\xi_{-}}\right| \leq \operatorname{Cte} \frac{(1+|\tau|)}{|\operatorname{Im} \tau|}, \\
x^{\prime} \geq 0 & \Rightarrow \operatorname{Im} \phi_{\varepsilon} \geq 0
\end{aligned}
$$

et on conclut par des estimations $L^{\infty}$ brutales.

Pour II on remarque qu'on a les estimations

$$
\left\|\partial_{x}^{k} \partial_{x^{\prime}}^{k^{\prime}} \psi(x) \sigma^{i} \psi\left(x^{\prime}\right) 1_{|\xi| \geq C_{0}(1+|\tau|)}\right\|_{L_{x, x^{\prime}, \xi, \tau}^{\infty}} \leq C_{k, k^{\prime}}
$$

et le cas $K_{\tau}^{1,+}$ résulte du lemme classique de continuité $L^{2}$ suivant.

Lemme 2.4. Si $q\left(x, x^{\prime}, \xi\right)$ est à support compact en $x, x^{\prime}$ et vérifie $\left\|\partial_{x}^{k} \partial_{x^{\prime}}^{k} q\left(x, x^{\prime}, \xi\right)\right\|_{L^{\infty}} \leq C_{k, k^{\prime}}$, lo.p.d. de symbole $q$ est continu sur $L^{2}(\mathbb{R})$, et sa norme est estimée par les $C_{k, k^{\prime}}$ pour $k \leq 2, k^{\prime} \leq 2,(x \in \mathbb{R})$.

Pour le cas de $K_{\tau}^{2,+}$ on remarque qu'il existe $c_{0}>0$ et pour tout $k, C_{k}$ tels que $x^{\prime} \geq 0$ et $|\xi| \geq C_{0}(1+|\tau|)$ entraîne $\operatorname{Im} \phi_{\varepsilon} \geq c_{0}\left(x^{\prime}|\xi|+\varepsilon|\xi|\right),\left|\partial_{x}^{k} \phi_{\varepsilon}\right| \leq$ $C_{k}\left(x^{\prime}+\varepsilon\right)|\xi|$ sur le support de $\psi\left(x^{\prime}\right)$. On a donc les estimées

$$
\begin{gathered}
\forall k, \quad \exists C_{k}, \quad \forall x^{\prime} \geq 0, \\
\sup _{x, \tau}\left|\partial_{x}^{k}\left(e^{i \phi_{\varepsilon}} \psi(x) \sigma^{2} \psi\left(x^{\prime}\right) 1_{|\xi| \geq C_{0}(1+|\tau|)}\right)\right| \leq C_{k} e^{-c_{0}\left(x^{\prime}+\varepsilon\right)|\xi| / 2},
\end{gathered}
$$

et on conclut en utilisant le: 
Lemme 2.5. Soit $q_{\varepsilon}(x, y, \xi)$ à support compact en $(x, y)$ vérifiant

$$
\forall k, \quad \exists C_{k}, \quad \forall y \geq 0, \quad \sup _{x}\left|\partial_{x}^{k} q_{\varepsilon}\right| \leq C_{k} e^{-c_{0}(y+\varepsilon)|\xi| / 2} .
$$

Alors lopérateur $Q_{\varepsilon} f(x)=\int e^{i(x-y) \xi} q_{\varepsilon}(x, y, \xi) f(y) d y d \xi$ est borné de $L^{2}\left(\mathbb{R}_{+}\right)$ dans $L^{2}(\mathbb{R})$, et sa norme est estimée, uniformément en $\varepsilon>0$, par $c_{0}, C_{k}$, $k \leq 2$.

Preuve. On a, pour un $\theta \in C_{0}^{\infty}$ et $R$ grand

$$
q_{\varepsilon}=\theta(x) \sum_{n} q_{\varepsilon, n}(y, \xi) e^{i n x / R}
$$

avec

$$
\left|q_{\varepsilon, n}\right| \leq C \exp \left(-\frac{c_{0}}{2}|\xi|(\varepsilon+y)\right) \frac{1}{1+n^{2}} \leq C \exp \left(-\frac{c_{0}}{2} y|\xi|\right) \frac{1}{1+n^{2}},
$$

la constante ne dépendant que de $c_{0}, C_{2}$. Il suffit donc de vérifier que l'opérateur de $L^{2}\left(\mathbb{R}_{+}\right)$dans $L^{2}\left(\mathbb{R}_{\xi}\right)$

$$
f \mapsto \int_{0}^{\infty} \exp \left(-\frac{c_{0}}{2} y|\xi|\right) \cdot f(y) d y
$$

est borné, ce qui résulte de l'inégalité de Hardy prouvée à la Proposition 1.1 et de la formule de Plancherel. Le Lemme 2.3 est alors démontré.

On définit de même les opérateurs $K_{\tau}^{i,-}$; avec les notations du $\S 2.2$, l'égalité (2.22) entraîne

$$
\left\{\begin{array}{l}
\psi \partial_{+} \varphi_{2} \underline{u}=\psi \partial_{+} \varphi_{2} u_{i}-\varphi_{2} K^{1,+}\left(\varphi_{1} v_{+}\right)-\varphi_{2} K^{2,-}\left(\varphi_{1} v_{-}\right), \\
\psi \partial_{-} \varphi_{2} \underline{u}=\psi \partial_{-} \varphi_{2} u_{i}-\varphi_{2} K^{2,+}\left(\varphi_{1} v_{+}\right)-\varphi_{2} K^{1,-}\left(\varphi_{1} v_{-}\right) .
\end{array}\right.
$$

Comme $\varphi_{1} v_{ \pm} \in E_{0}$, il résulte du Lemme 2.3 que $\varphi_{2} K^{1,+}\left(\varphi_{1} v_{+}\right) \in E_{1}$. On a aussi $\psi \partial_{ \pm} \varphi_{2} u_{i} \in L^{2}$. Soit $\theta(x) \in C^{\infty}, \theta(x)=0$ pour $x \leq 1, \theta(x)=1$ pour $x \geq 2$. En multipliant (2.29) par $\theta(x / \varepsilon)$, et en faisant tendre $\varepsilon$ vers 0 on obtient donc

$$
\left\{\begin{array}{l}
\psi \varphi_{2} v_{+}=h_{+}^{0}-\varphi_{2} 1_{x \geq 0} K^{1,+}\left(\varphi_{1} v_{+}\right)-\varphi_{2} 1_{x \geq 0} K^{2,-}\left(\varphi_{1} v_{-}\right), \\
\psi \varphi_{2} v_{-}=h_{-}^{0}-\varphi_{2} 1_{x \geq 0} K^{2,+}\left(\varphi_{1} v_{+}\right)-\varphi_{2} 1_{x \geq 0} K^{1,-}\left(\varphi_{1} v_{-}\right),
\end{array}\right.
$$

avec

$$
h_{ \pm}^{0}=1_{x \geq 0} \psi \varphi_{2} \partial_{ \pm} u_{i}
$$

On pose à présent

$$
\left\{\begin{array}{l}
K^{1, \pm}=\left(-\frac{1}{2} \psi(x) \otimes \psi\left(x^{\prime}\right)-\frac{1}{2} Q^{ \pm}\right) \circ 1_{x^{\prime} \geq 0}, \\
1_{x \geq 0} K^{2,+}=-\frac{1}{2} S^{-,+}, \quad 1_{x \geq 0} K^{2,-}=-\frac{1}{2} S^{+,-},
\end{array}\right.
$$

et on introduit les inconnues

$$
g_{ \pm}=\psi \varphi_{1} v_{ \pm}=\varphi_{1} v_{ \pm} .
$$


Alors (2.30) se réécrit sous la forme

$$
\begin{gathered}
\left\{\begin{array}{l}
g_{+}-1_{x \geq 0} Q^{+}\left(g_{+}\right)-S^{+,-}\left(g_{-}\right)=2 h_{+}^{0}+r_{+}, \\
g_{-}-S^{-,+}\left(g_{+}\right)-1_{x \geq 0} Q^{-}\left(g_{-}\right)=2 h_{-}^{0}+r_{-},
\end{array}\right. \\
\left\{\begin{array}{l}
r_{+}=\left(1-\varphi_{2}\right) g_{+}-\left(1-\varphi_{2}\right)\left[1_{x \geq 0} Q^{+}\left(g_{+}\right)+S^{+,-}\left(g_{-}\right)\right], \\
r_{-}=\left(1-\varphi_{2}\right) g_{-}-\left(1-\varphi_{2}\right)\left[S^{-,+}\left(g_{+}\right)+1_{x \geq 0} Q^{-}\left(g_{-}\right)\right],
\end{array}\right.
\end{gathered}
$$

d'où il résulte que $r_{ \pm} \in E_{1}$, est à support compact en $x$, et à support dans $t \geq t_{0}$. De plus, les opérateurs $Q_{\tau}^{ \pm}, S_{\tau}^{+,-}, S_{\tau}^{-,+}$sont donnés par

$$
\begin{aligned}
& Q_{\tau}^{+}(f)(x) \\
& \quad=\frac{1}{2 \pi} \int e^{i\left(x-x^{\prime}\right) \xi}\left(\frac{\xi}{\xi_{+}}-\mu\right)\left(\frac{\mu-a^{\prime}(x)}{1+\mu^{2}}\right) \psi(x) \psi\left(x^{\prime}\right) f\left(x^{\prime}\right) d x^{\prime} d \xi, \\
& Q_{\tau}^{-}(f)(x) \\
& \quad=\frac{1}{2 \pi} \int e^{i\left(x-x^{\prime}\right) \xi}\left(\frac{\xi}{\xi_{-}}+\nu\right)\left(\frac{b^{\prime}(x)-\nu}{1+\nu^{2}}\right) \psi(x) \psi\left(x^{\prime}\right) f\left(x^{\prime}\right) d x^{\prime} d \xi
\end{aligned}
$$

$$
\begin{aligned}
S_{\tau}^{+,-} & (f)(x) \\
& =\frac{1}{2 \pi} \int e^{i \psi_{+,-}\left(x, x^{\prime}, \xi, \tau\right)}\left(1-a^{\prime}(x) \frac{\xi}{\xi_{0}}\right) 1_{x \geq 0, x^{\prime} \geq 0} \psi(x) \psi\left(x^{\prime}\right) f\left(x^{\prime}\right) d x^{\prime} d \xi,
\end{aligned}
$$

$$
\begin{aligned}
S_{\tau}^{-,+} & (f)(x) \\
& =\frac{1}{2 \pi} \int e^{i \psi_{-,+}\left(x, x^{\prime}, \xi, \tau\right)}\left(1+b^{\prime}(x) \frac{\xi}{\xi_{0}}\right) 1_{x \geq 0, x^{\prime} \geq 0} \psi(x) \psi\left(x^{\prime}\right) f\left(x^{\prime}\right) d x^{\prime} d \xi,
\end{aligned}
$$

avec

$$
\begin{aligned}
\xi_{0} & =\left(\tau^{2}-\xi^{2}\right)^{1 / 2}, \quad \operatorname{Im} \xi_{0}>0, \\
\psi_{+,-} & =\left(x-x^{\prime}\right) \xi+\left(a(x)-b\left(x^{\prime}\right)\right) \xi_{0}, \\
\psi_{-,+} & =\left(x-x^{\prime}\right) \xi+\left(a\left(x^{\prime}\right)-b(x)\right) \xi_{0} .
\end{aligned}
$$

En effet (2.36) et (2.37) résultent directement de la définition (2.32) de $Q^{ \pm}$, de (2.27), et du calcul analogue de $Q^{-}$. Pour prouver l'identité (2.39) il suffit de vérifier que:

(i) pour $\tau \in \mathbb{C}^{-}$, l'opérateur défini par (2.39) est borné sur $L^{2}(\mathbb{R})$, et vérifie l'estimation (2.17) avec $e=1$.

(ii) L'opérateur défini par (2.39) coïncide avec $S_{\tau}^{-,+}$sur les $f \in C_{0}^{\infty}\left(\mathbb{R}_{+}^{*}\right)$.

Or (ii) résulte d'un calcul direct à partir des définitions (2.24) et (2.32) des opérateurs. Pour (i), on coupe à nouveau l'intégrale en $\xi$ en deux morceaux, I: $|\xi| \leq C_{0}(1+|\tau|)$, II: $|\xi| \geq C_{0}(1+|\tau|)$ avec $C_{0}$ grand. 
Pour I, on remarque que $x \geq 0$ et $x^{\prime} \geq 0$ entraîne $\operatorname{Im} \psi_{+,-} \geq 0$ et

$$
|\xi| \leq C_{0}(1+|\tau|) \Rightarrow \frac{|\xi|}{\left|\xi_{0}\right|} \leq \operatorname{Cte} \frac{1+|\tau|}{|\operatorname{Im} \tau|} .
$$

Pour II, on remarque qu'il existe $c_{0}>0$ tel que

$$
\begin{aligned}
|\xi| \geq & C_{0}(1+|\tau|) \\
& \Rightarrow\left|e^{i \psi_{+,-}}\left(1-a^{\prime}(x) \frac{\xi}{\xi_{0}}\right) \psi(x) \psi\left(x^{\prime}\right) 1_{x \geq 0, x^{\prime} \geq 0}\right| \leq e^{-c_{0}\left(x+x^{\prime}\right)|\xi|},
\end{aligned}
$$

et l'opérateur

$$
\int_{0}^{\infty} d y \int d \xi e^{-c_{0}(x+y)|\xi|} f(y)=\frac{2}{c_{0}} \int_{0}^{\infty} \frac{f(y)}{x+y} d y
$$

est borné sur $L^{2}\left(\mathbb{R}_{+}\right)$.

L'identité (2.38) se prouve de manière analogue.

Terminons cette section en décrivant les coordonnées sur les cotangents aux courbes

$$
\widetilde{\Delta}_{+}=\{(t, x, a(x)), t \in \mathbb{R}, x \in \mathbb{R}\}
$$

et

$$
\tilde{\Delta}_{-}=\{(t, x, b(x)), t \in \mathbb{R}, x \in \mathbb{R}\} .
$$

Le choix des coordonnées $(t, x)$ sur ces courbes donne lieu à la description suivante des applications canoniques $j_{ \pm}:\left.T^{*} \mathbb{R}^{3}\right|_{\widetilde{\Delta}_{ \pm}} \rightarrow T^{*} \widetilde{\Delta}_{ \pm}$,

$$
\begin{aligned}
& j_{+}(t, x, a(x), \tau, \xi, \eta)=\left(t, x, \tau, \xi+a^{\prime}(x) \eta\right), \\
& j_{-}(t, x, b(x), \tau, \xi, \eta)=\left(t, x, \tau, \xi+b^{\prime}(x) \eta\right) .
\end{aligned}
$$

Rappelons (voir $[\mathrm{H}]$ ) que les zones glancing $\mathscr{G}$, hyperboliques $\mathscr{H}$ et elliptiques $\mathscr{E}$ pour $p=\tau^{2}-\xi^{2}-\eta^{2}$ sur $T^{*} \widetilde{\Delta}_{ \pm}$sont données par les conditions suivantes sur le système $S_{\tilde{\rho}}^{ \pm}: p(\tilde{\rho})=0, j_{ \pm}(\tilde{\rho})=\rho$;

$$
\begin{aligned}
\rho \in \mathscr{G}^{ \pm} & \Leftrightarrow \quad S_{\rho}^{ \pm} \text {a une solution double, } \\
\rho \in \mathscr{H}^{ \pm} & \Leftrightarrow \quad S_{\tilde{\rho}}^{ \pm} \text {a deux solutions distinctes, } \\
\rho \in \mathscr{E}^{ \pm} & \Leftrightarrow \quad S_{\tilde{\rho}}^{ \pm} \text {n'a pas de solution. }
\end{aligned}
$$

En utilisant les coordonnées sur $T^{*} \widetilde{\Delta}_{+}$héritées de $(t, x)$, on en déduit que $(t, x, \tau, \tilde{\xi}) \in \mathscr{G}^{+}\left(\right.$resp. $\left.\mathscr{H}^{+}, \mathscr{E}^{+}\right)$si et seulement si la quantité $\tilde{\xi}^{2}-$ $\left(1+a^{\prime}(x)^{2}\right) \tau^{2}$ est nulle (resp. négative, positive). On a une description analogue sur $T^{*} \widetilde{\Delta}_{-}$, en changeant $a$ en $b$.

Compte tenu de la formule (2.7) et du théorème de propagation des singularités pour $\square$, il suffit de décrire $v_{ \pm}$microlocalement près de $t=0, x=0$, 
$\tau \neq 0, \tilde{\xi} \neq \frac{-\tau}{\cos \gamma_{ \pm}}$pour décrire $u$ sur l'ouvert $\Omega^{\varepsilon} \cap\left\{0<t<\varepsilon_{1}\right\}$ pour $\varepsilon_{1}>0$ assez petit. Tel est le but des sections suivantes.

\section{RÉDUCTION DEUX-MICROLOCALE}

3.1. Opérateurs pseudo-différentiels dans le domaine complexe pour les problèmes aux limites. On note $I$ l'intervalle $\left[0, t_{0}\right]$, où $t_{0}>0, U$ un ouvert de $T^{*} \mathbb{C}^{d}$, et $K$ un compact $\mathbb{C} \backslash \mathbb{R}$.

Definition 3.1. Pour $m \in \mathbb{Z}$, on note $A^{m}(I, U, K)$ l'espace des séries formelles

$$
e=\sum_{n \geq 0} \frac{1}{(i \lambda)^{n}} e_{n}
$$

pour lesquelles il existe $V$ voisinage complexe de $I$, et $A, B>0$ tels que pour tout $n$, la fonction $e_{n}(t, \tau, z, \zeta, \lambda)$ soit définie pour $\lambda \geq 1$, holomorphe en $t \in V, \tau \in \mathbb{C} \backslash K,(z, \zeta) \in U$ et vérifie

$$
\left|e_{n}(t, \tau, z, \zeta, \lambda)\right| \leq A B^{n} n !(1+|\tau|)^{m} .
$$

Pour $e \in A^{m}(I, U, K)$ les $e_{n}$ sont méromorphes en $\tau$ à l'infini, et, si $m_{+}=\sup (m, 0)$, on a une décomposition unique

$$
e_{n}(t, \tau, z, \zeta, \lambda)=\sum_{j=0}^{m_{+}} e_{n}^{j}(t, z, \zeta, \lambda) \tau^{j}+e_{n}^{-}(t, \tau, z, \zeta, \lambda),
$$

où $e^{-} \stackrel{\text { def }}{=} \sum_{n \geq 0}(i \lambda)^{-n} e_{n}^{-} \in A^{-1}(I, U, K)$ et où $e^{j} \stackrel{\text { def }}{=} \sum_{n \geq 0}(i \lambda)^{-n} e_{n}^{j} \in$ $A^{0}(I, U, K)$ est indépendant de $\tau$.

Pour $\zeta=\left(\zeta_{1}, \ldots, \zeta_{d}\right) \in \mathbb{C}^{d}$, on posera $\|\zeta\|=\sup _{1 \leq j \leq d}\left|\zeta_{j}\right|$ et, pour $\zeta_{0} \in$ $\mathbb{C}^{d}, R>0$,

$$
B\left(\zeta_{0}, R\right)=\left\{\zeta \in \mathbb{C}^{d},\left\|\zeta-\zeta_{0}\right\|<R\right\}
$$

Soient $\Omega$ un ouvert de $\mathbb{C}^{d}, \zeta_{0} \in \mathbb{C}^{d}$, et $R_{0}>0$ tels que $\Omega \times B\left(\zeta_{0}, R_{0}\right) \subset U$. Pour $e \in A^{m}(I, U, K)$, on a, par les inégalités de Cauchy,

$\forall t \in V, \quad \forall \tau \in \mathbb{C} \backslash K, \quad \forall z \in \Omega$,

$$
\left|\partial_{\zeta}^{\alpha} e_{n}\left(t, \tau, z, \zeta_{0}, \lambda\right)\right| \leq A \frac{\alpha !}{R_{0}^{|\alpha|}} B^{n} n !(1+|\tau|)^{m}
$$

et on pose

$$
k\left(e, \zeta_{0} ; n, \alpha\right)(t, s, z, \lambda)=\frac{\lambda}{2 \pi} \int_{-\infty}^{+\infty} e^{i \lambda(t-s) \tau} \frac{1}{\alpha !} \partial_{\zeta}^{\alpha} e_{n}\left(t, \tau, z, \zeta_{0}, \lambda\right) d \tau
$$

où l'intégrale est oscillante. Si on pose $K_{ \pm}=K \cap\{ \pm \operatorname{Im} \tau>0\}$ et

$$
k^{ \pm}\left(e, \zeta_{0} ; n, \alpha\right)(t, s, z, \lambda)=\left.k\left(e, \zeta_{0} ; n, \alpha\right)(t, s, z, \lambda)\right|_{ \pm(t-s)>0},
$$

alors $k^{ \pm}$est $C^{\infty}$ dans $\pm(t-s) \geq 0$, holomorphe en $z \in \Omega$, et vérifie

$$
\forall \beta, \quad \exists C_{\beta}, \quad \forall(t, s) \in I^{2}, \quad \pm(t-s) \geq 0, \quad \forall z \in \Omega, \quad \forall \lambda \geq 1,
$$




$$
\begin{aligned}
& \left|\partial_{s, t}^{\beta} k^{ \pm}\left(e, \zeta_{0} ; n, \alpha\right)(t, s, z, \lambda)\right| \\
& \quad \leq C_{\beta} \lambda^{|\beta|+1} B^{n} R_{0}^{-|\alpha|} n ! e^{-(\lambda / 2)|t-s| \operatorname{dist}\left(K_{ \pm}, \mathbb{R}\right)}
\end{aligned}
$$

et le noyau $k$ est donné par

$$
\begin{aligned}
k\left(e, \zeta_{0} ; n, \alpha\right)= & 1_{t \geq s} k^{+}\left(e, \zeta_{0} ; n, \alpha\right)+1_{t \leq s} k^{-}\left(e, \zeta_{0} ; n, \alpha\right) \\
& +\sum_{n=0}^{m_{+}} \frac{1}{\alpha !} \partial_{\zeta}^{\alpha} e_{n}^{j}\left(t, z, \zeta_{0}, \lambda\right)(i \lambda)^{-j}\left(\frac{\partial}{\partial t}\right)^{j}\left(\delta_{t=s}\right) .
\end{aligned}
$$

Définition 3.2. Soit $\varphi \in C^{1}(\Omega, \mathbb{R})$. On désigne par $C^{\infty}\left(I, H_{\varphi}(\Omega)\right)$ l'espace des fonctions $u(t, z, \lambda)$ définies pour $\lambda \geq 1$, holomorphes en $z \in \Omega, C^{\infty}$ en $t \in I$, telles que

$$
\exists M, \quad \forall l, \quad \exists C_{l}, \quad \forall(t, z, \lambda), \quad\left|\partial_{t}^{l} u(t, z, \lambda)\right| \leq C_{l} \lambda^{M+l} e^{\lambda \varphi(z)} .
$$

On identifie $\mathbb{C}^{d}$ à l'espace des formes $\mathbb{R}$-linéaires de $\mathbb{C}^{d}$ dans $\mathbb{R}$ par $\zeta \mapsto$ $(w \mapsto-\operatorname{Im}(\zeta \cdot w))$. On a en particulier

$$
d \varphi(z)(w)=-\operatorname{Im}\left(\frac{2}{i} \frac{\partial \varphi}{\partial z}(z) \cdot w\right) .
$$

Définition 3.3. Soient $e \in A^{m}(I, U, K)$, avec $\Omega \times B\left(\zeta_{0}, R_{0}\right) \subset U$, et $C_{1}, C_{2}$ deux constantes positives. Pour $u \in C^{\infty}\left(I, H_{\varphi}(\Omega)\right)$, on pose, pour $t \in I$, $z \in \Omega, \lambda \geq 1$,

$$
\begin{aligned}
\operatorname{Op}(e) & \left(\zeta_{0}, C_{1}, C_{2}\right)(u)(t, z, \lambda) \\
= & \sum_{n \leq \lambda / C_{1}} \sum_{|\alpha| \leq \lambda / C_{2}}(i \lambda)^{-n} \int_{0}^{t_{0}} k\left(e, \zeta_{0} ; n, \alpha\right)(t, s, z, \lambda) u_{\alpha}^{\zeta_{0}}(s, z, \lambda) d s,
\end{aligned}
$$

où

$$
u_{\alpha}^{\zeta_{0}}(s, z, \lambda)=\left(\frac{1}{i \lambda} \partial_{z}-\zeta_{0}\right)^{\alpha} u(s, z, \lambda)=e^{i \lambda z \zeta_{0}}\left(\frac{1}{i \lambda} \partial_{z}\right)^{\alpha}\left(e^{-i \lambda z \zeta_{0}} u(s, z, \lambda)\right)
$$

$$
\begin{aligned}
\int_{0}^{t_{0}} k(t, s) v(s) d s= & \int_{0}^{t} k^{+}(t, s) v(s) d s+\int_{t}^{t_{0}} k^{-}(t, s) v(s) d s \\
& +\sum_{n=0}^{m_{+}}(i \lambda)^{-j} \frac{1}{\alpha !} \partial_{\zeta}^{\alpha} e_{n}^{j}(t) \partial_{t}^{j} v(t) .
\end{aligned}
$$

On remarquera que l'opérateur $\operatorname{Op}(e)\left(\zeta_{0}, C_{1}, C_{2}\right)$ est différentiel en $z$ et que $\mathrm{Op}(1)=\mathrm{Id}$.

Lemme 3.1. Soit $D>0$ tel que

$$
\sup _{z \in \Omega}\left\|\frac{2}{i} \frac{\partial \varphi}{\partial z}(z)-\zeta_{0}\right\|<D
$$


On suppose qu'on a $D<R_{0}$. Soient $\Omega^{\prime} \Subset \Omega$, et $C_{0}=\left(D \operatorname{dist}\left(\bar{\Omega}^{\prime}, \Omega^{c}\right)\right)^{-1}$ (où $\operatorname{dist}(a, b)=\|a-b\|)$. Pour $C_{1} \geq 2 B / e, C_{2} \geq C_{0}, u \in C^{\infty}\left(I, H_{\varphi}(\Omega)\right)$, on $a$

$$
\mathrm{Op}(e)\left(\zeta_{0}, C_{1}, C_{2}\right)(u) \in C^{\infty}\left(I, H_{\varphi}\left(\Omega^{\prime}\right)\right) .
$$

De plus, si $C_{1}, C_{1}^{\prime} \geq 2 B / e, C_{2}, C_{2}^{\prime} \geq C_{0}$ il existe $\varepsilon>0$ tel que

$$
\operatorname{Op}(e)\left(\zeta_{0}, C_{1}, C_{2}\right)(u)-\mathrm{Op}(e)\left(\zeta_{0}, C_{1}^{\prime}, C_{2}^{\prime}\right)(u) \in C^{\infty}\left(I, H_{\varphi-\varepsilon}\left(\Omega^{\prime}\right)\right)
$$

pour tout $u \in C^{\infty}\left(I, H_{\varphi}(\Omega)\right)$.

Démonstration. D'après le choix de $C_{0}$, si $z \in \Omega^{\prime}$ et $\|w-z\| \leq\left(D C_{0}\right)^{-1}$ on a $w \in \Omega$. Pour $|\alpha| \leq \lambda / C_{0}$ posons $\rho_{\alpha_{j}}=\alpha_{j}(\lambda D)^{-1}$. Alors, si $z \in \Omega^{\prime}$ et $\left|w_{j}-z_{j}\right| \leq \rho_{\alpha_{j}}$, on a $w \in \Omega$. En appliquant les inégalités de Cauchy à la fonction $u e^{-i \lambda z \zeta_{0}}$, on obtient

$$
\begin{aligned}
& \sup _{z \in \Omega^{\prime}}\left|u_{\alpha}^{\zeta_{0}}(s, z, \lambda) e^{-\lambda \varphi(z)}\right| \\
& \quad \leq \frac{\alpha ! \lambda^{-|\alpha|}}{\prod_{j=1}^{d} \rho_{\alpha_{j}}^{\alpha_{j}}} \sup _{w \in \Omega}\left|u(s, w, \lambda) e^{-\lambda \varphi(w)}\right|_{\left|w_{j}-z_{j}\right|=\rho_{\alpha_{j}}}\left|e^{\lambda\left(\varphi(w)-\varphi(z)+i(z-w) \zeta_{0}\right)}\right| .
\end{aligned}
$$

Or

$$
\begin{aligned}
& \operatorname{Re}\left(\varphi(w)-\varphi(z)+i(z-w) \zeta_{0}\right) \\
& \quad=-\operatorname{Im}\left(\int_{0}^{1}\left(\frac{2}{i} \frac{\partial \varphi}{\partial z}(z+\theta(w-z))-\zeta_{0}\right) d \theta \cdot(w-z)\right) \leq \frac{|\alpha|}{\lambda}
\end{aligned}
$$

En utilisant la formule de Stirling, on en déduit l'estimation suivante:

$$
\begin{aligned}
\forall l, \quad \exists C_{l}, \quad \forall \alpha, \quad|\alpha| & \leq \lambda / C_{0}, \quad \forall s \in I, \\
\sup _{z \in \Omega^{\prime}}\left|\partial_{s}^{l} u_{\alpha}^{\zeta_{0}}(s, z, \lambda) e^{-\lambda \varphi(z)}\right| & \leq C_{l} \lambda^{M+l} \alpha ! \lambda^{-|\alpha|} e^{|\alpha|} \prod_{j=1}^{d} \rho_{\alpha_{j}}^{-\alpha_{j}} \\
& \leq C_{l}^{\prime} \lambda^{M+l} D^{|\alpha|} \prod_{j=1}^{d}\left(\alpha_{j}+1\right)^{1 / 2} .
\end{aligned}
$$

Le lemme résulte alors de (3.5), (3.8), (3.15), puisque

$$
\sum_{n \leq \lambda / C_{1}} \lambda^{-n} B^{n} n ! \sum_{|\alpha| \leq \lambda / C_{2}}\left(\frac{D}{R_{0}}\right)^{|\alpha|} \leq \operatorname{Cte} \sum_{n=0}^{\infty}\left(\frac{B}{e C_{1}}\right)^{n} \sqrt{n+1}<+\infty,
$$

et, si $\gamma<1$,

$$
\sum_{\lambda / C^{\prime} \leq n \leq \lambda / C} \gamma^{n} \leq \mathrm{Cte} e^{-\lambda|\ln \gamma| / 2 C^{\prime}} .
$$

Lemme 3.2. On conserve les notations du Lemme 3.1, et on se donne un autre triplet $\zeta_{0}^{\prime}, R_{0}^{\prime}, D^{\prime}$ tel que

$$
\begin{gathered}
\Omega \times B\left(\zeta_{0}^{\prime}, R_{0}^{\prime}\right) \subset U, \\
\sup _{z \in \Omega}\left\|\frac{2}{i} \frac{\partial \varphi}{\partial z}(z)-\zeta_{0}^{\prime}\right\|<D^{\prime}<R_{0}^{\prime} .
\end{gathered}
$$


Soit $C_{0}^{\prime}=\left(D^{\prime} \operatorname{dist}\left(\bar{\Omega}^{\prime}, \Omega^{c}\right)\right)^{-1} ;$ alors pour tous $C_{1} \geq 2 B / e, C_{1}^{\prime} \geq 2 B / e, C_{2} \geq$ $C_{0}, C_{2}^{\prime} \geq C_{0}^{\prime}$, il existe $\varepsilon>0$ tel que

$$
\operatorname{Op}(e)\left(\zeta_{0}, C_{1}, C_{2}\right)(u)-\mathrm{Op}(e)\left(\zeta_{0}^{\prime}, C_{1}^{\prime}, C_{2}^{\prime}\right)(u) \in C^{\infty}\left(I, H_{\varphi-\varepsilon}\left(\Omega^{\prime}\right)\right)
$$

pour tout $u \in C^{\infty}\left(I, H_{\varphi}(\Omega)\right)$.

Démonstration. Il suffit de prouver que, pour tout $z_{0} \in \bar{\Omega}^{\prime}$, il existe $r>0$ tel que

$$
\operatorname{Op}(e)\left(\zeta_{0}, C_{1}, C_{2}\right)(u)-\mathrm{Op}(e)\left(\zeta_{0}, C_{1}^{\prime}, C_{2}^{\prime}\right)(u) \in C^{\infty}\left(I, H_{\varphi-\varepsilon}\left(B\left(z_{0}, r\right)\right)\right) .
$$

Posons $\zeta_{0}^{\prime \prime}=2 \partial_{z} \varphi\left(z_{0}\right) / i$ et soit $R_{0}^{\prime \prime}$ tel que $B\left(\zeta_{0}^{\prime \prime}, 2 R_{0}^{\prime \prime}\right) \subset B\left(\zeta_{0}, R_{0}\right) \cap B\left(\zeta_{0}^{\prime}, R_{0}^{\prime}\right)$. Il existe alors $r>0$ tel que $B\left(z_{0}, 2 r\right) \times B\left(\zeta_{0}^{\prime \prime}, R_{0}^{\prime \prime}\right) \subset U$ et

$$
\sup _{z \in B\left(z_{0}, 2 r\right)}\left\|\frac{2}{i} \frac{\partial \varphi}{\partial z}(z)-\zeta_{0}^{\prime \prime}\right\|<D^{\prime \prime}<R_{0}^{\prime \prime} .
$$

D'après le Lemme 3.1 on peut, en augmentant $C_{2}$ et $C_{2}^{\prime}$, remplacer $\Omega$ par $B\left(z_{0}, 2 r\right), \Omega^{\prime}$ par $B\left(z_{0}, r\right)$, et supposer que

$$
C_{2}=C_{2}^{\prime}>\max \left((D r)^{-1},\left(D^{\prime} r\right)^{-1},\left(D^{\prime \prime} r\right)^{-1}\right) \text {. }
$$

On peut alors aussi remplacer $\zeta_{0}^{\prime}$ par $\zeta_{0}^{\prime \prime}$. Posons

$$
I_{n}=\sum_{|\alpha| \leq \lambda / C_{2}} \int_{0}^{t_{0}} k\left(e, \zeta_{0} ; n, \alpha\right) u_{\alpha}^{\zeta_{0}}-k\left(e, \zeta_{0}^{\prime \prime} ; n, \alpha\right) u_{\alpha}^{\zeta_{0}^{\prime \prime}} d s
$$

En utilisant la décomposition (3.9) du noyau $k$, on a

$$
I_{n}=I_{n}^{+}+I_{n}^{-}+\sum_{j=0}^{m_{+}} I_{n}^{j} \text {. }
$$

Si $\gamma_{+} \subset\{\operatorname{Im} \tau>0\}$ entoure $K_{+}$une fois dans le sens positif, on a

$$
\begin{aligned}
I_{n}^{+}=\frac{\lambda}{2 \pi} \int_{0}^{t} d s & \int_{\gamma_{+}} d \tau \sum_{|\alpha| \leq \lambda / C_{2}} e^{i \lambda \tau(t-s)} \frac{1}{\alpha !} \\
& \times\left(\partial_{\zeta}^{\alpha} e_{n}\left(\zeta_{0}\right)\left(\partial_{z} / i \lambda-\zeta_{0}\right)^{\alpha} u-\partial_{\zeta}^{\alpha} e_{n}\left(\zeta_{0}^{\prime \prime}\right)\left(\partial_{z} / i \lambda-\zeta_{0}^{\prime \prime}\right)^{\alpha} u\right) .
\end{aligned}
$$

Or

$$
\begin{aligned}
\left(\partial_{z} / i \lambda-\zeta_{0}\right)^{\alpha} & =\sum_{\alpha_{1}+\alpha_{2}=\alpha} \frac{\alpha !}{\alpha_{1} ! \alpha_{2} !}\left(\zeta_{0}^{\prime \prime}-\zeta_{0}\right)^{\alpha_{2}}\left(\partial_{z} / i \lambda-\zeta_{0}^{\prime \prime}\right)^{\alpha_{1}} \\
\partial_{\zeta}^{\alpha_{1}} e_{n}\left(\zeta_{0}^{\prime \prime}\right) & =\sum_{\alpha_{2}} \frac{1}{\alpha_{2} !} \partial_{\zeta}^{\alpha_{1}+\alpha_{2}} e_{n}\left(\zeta_{0}\right)\left(\zeta_{0}^{\prime \prime}-\zeta_{0}\right)^{\alpha_{2}}
\end{aligned}
$$

Soit $\gamma=\left\|\zeta_{0}^{\prime \prime}-\zeta_{0}\right\| / R_{0}<1$. On a, pour tout $z \in B\left(z_{0}, r\right)$,

$$
I_{n}^{+}=-\frac{\lambda}{2 \pi} \int_{0}^{t} d s \int_{\gamma_{+}} d \tau e^{i \lambda \tau(t-s)} \sum_{E} \frac{1}{\alpha_{1} ! \alpha_{2} !} \partial_{\zeta}^{\alpha_{1}+\alpha_{2}} e_{n}\left(\zeta_{0}\right)\left(\zeta_{0}^{\prime \prime}-\zeta_{0}\right)^{\alpha_{2}} u_{\alpha_{1}}^{\zeta_{0}^{\prime \prime}}
$$


où $E=\left\{\left(\alpha_{1}, \alpha_{2}\right),\left|\alpha_{1}\right| \leq \lambda / C_{2},\left|\alpha_{1}+\alpha_{2}\right|>\lambda / C_{2}\right\}$. Alors, d'après (3.5), (3.15) et $(3.20)$,

$$
\begin{aligned}
& \forall l, \quad \exists C_{l}, \quad\left|\partial_{t}^{l} I_{n}^{+}(t, z, \lambda)\right| e^{-i \varphi(z)} \\
& \leq C_{l} \lambda^{M+l} B^{n} n ! \sum_{E} \frac{\left(\alpha_{1}+\alpha_{2}\right) !}{\alpha_{1} ! \alpha_{2} !} \frac{\left(\gamma R_{0}\right)^{\left|\alpha_{2}\right|}}{R_{0}^{\left|\alpha_{1}+\alpha_{2}\right|}} D^{\prime \prime\left|\alpha_{1}\right|} \prod_{j=1}^{d}\left(\alpha_{1, j}+1\right)^{1 / 2} .
\end{aligned}
$$

Comme $D^{\prime \prime}<R_{0}^{\prime \prime}$, en traitant de même $I_{n}^{-}$et $I_{n}^{j}$, on obtient

$$
\begin{aligned}
\forall l, \quad \exists C_{l}, \quad \mid \partial_{t}^{l} I_{n}(t, & , z, \lambda) \mid e^{-\lambda \varphi(z)} \\
& \leq C_{l} \lambda^{M+l} B^{n} n ! \sum_{E} \frac{\left(\alpha_{1}+\alpha_{2}\right) !}{\alpha_{1} ! \alpha_{2} !} \gamma^{\left|\alpha_{2}\right|}\left(\frac{R_{0}^{\prime \prime}}{R_{0}}\right)^{\left|\alpha_{1}\right|} .
\end{aligned}
$$

Or on a

$$
\sum_{\alpha_{2}} \frac{\left(\alpha_{1}+\alpha_{2}\right) !}{\alpha_{1} ! \alpha_{2} !} \gamma^{\left|\alpha_{2}\right|}=\frac{1}{(1-\gamma)^{d+\left|\alpha_{1}\right|}}
$$

et $R_{0}^{\prime \prime} / R_{0}(1-\gamma)<1$. Par suite, dans (3.22), la somme restreinte à $\left|\alpha_{1}\right| \geq \lambda / 2 C_{2}$ est dominée par $e^{-\varepsilon \lambda}$. Pour le terme restant, on a $\left|\alpha_{2}\right| \geq \lambda / 2 C_{2}$ et le Lemme 3.2 résulte alors de l'inégalité

$$
\sum_{|\alpha| \geq a} \frac{\left(\alpha_{1}+\alpha_{2}\right) !}{\alpha_{1} ! \alpha_{2} !} \gamma^{\left|\alpha_{2}\right|} \leq C_{\theta} \frac{1}{\theta^{\left|\alpha_{1}\right|}} e^{-(a / 2) \log ((1-\theta) / \gamma)}
$$

avec $\gamma<1-\theta$; pour conclure, il suffit de choisir $\theta>R_{0}^{\prime \prime} / R_{0}$.

On s'intéresse à présent aux propriétés de composition des opérateurs, en se restreignant au cas qui va nous intéresser par la suite.

Soient $e \in A^{-2}(I, U, K)$ et $q \in A^{0}(I, U, K)$ tel que $q$ soit indépendant de $\tau$. On définit comme d'habitude le symbole composé $g=e \circ q$ par

$$
\begin{aligned}
g_{n}(t, \tau, z, \zeta, \lambda) & \\
& =\sum_{n^{\prime}+n^{\prime \prime}+|\alpha|+l=n} \frac{1}{l ! \alpha !} \partial_{\tau}^{l} \partial_{\zeta}^{\alpha} e_{n^{\prime}}(t, \tau, z, \zeta, \lambda) \partial_{t}^{l} \partial_{z}^{\alpha} q_{n^{\prime \prime}}(t, z, \zeta, \lambda) .
\end{aligned}
$$

Si $K_{1}$ est un voisinage compact de $K$ dans $\mathbb{C} \backslash \mathbb{R}$ et si $U_{1} \Subset U$, on a $e, g \in$ $A^{-2}\left(I, U_{1}, K_{1}\right)$ et $q \in A^{0}\left(I, U_{1}, K_{1}\right)$. Soient $\Omega, \zeta_{0}, R_{0}, D$ tels que $\Omega \times$ $B\left(\zeta_{0}, R_{0}\right) \subset U_{1}$ et

$$
\sup _{z \in \Omega}\left\|\frac{2}{i} \frac{\partial \varphi}{\partial z}(z)-\zeta_{0}\right\|<D<R_{0}
$$

Lemme 3.3. Soit $\Omega^{\prime} \Subset \Omega$. Il existe $C_{1}^{0}, C_{2}^{0}$ et, pour tous $C_{1} \geq C_{1}^{0}, C_{2} \geq C_{2}^{0}$, un $\varepsilon>0$, tels que, pour tout $u \in C^{\infty}\left(I, H_{\varphi}(\Omega)\right)$,

$$
\begin{aligned}
& \left(\mathrm{Op}(e \circ q)\left(\zeta_{0}, C_{1}, C_{2}\right)\right. \\
& \left.\quad-\operatorname{Op}(e)\left(\zeta_{0}, C_{1}, C_{2}\right) \operatorname{Op}(q)\left(\zeta_{0}, C_{1}, C_{2}\right)\right) u \in C^{\infty}\left(I, H_{\varphi-\varepsilon}\left(\Omega^{\prime}\right)\right) .
\end{aligned}
$$


Démonstration. En changeant $u$ en $u e^{-i \lambda z \cdot \zeta_{0}}$ et $\varphi$ en $\varphi+\operatorname{Im}\left(z \cdot \zeta_{0}\right)$, on peut supposer $\zeta_{0}=0$. On pose

$$
\begin{aligned}
e_{n}^{\alpha}(t, \tau, z, \lambda) & =\frac{1}{\alpha !} \partial_{\zeta}^{\alpha} e_{n}(t, \tau, z, 0, \lambda) \\
q_{n, l, \alpha}^{\beta}(t, z, \lambda) & =\frac{1}{l ! \beta ! \alpha !} \partial_{t}^{l} \partial_{z}^{\alpha} \partial_{\zeta}^{\beta} q_{n}(t, z, 0, \lambda), \\
g_{n}^{\gamma}(t, \tau, z, \lambda) & =\frac{1}{\gamma !} \partial_{\zeta}^{\gamma} g_{n}(t, \tau, z, 0, \lambda) \\
u_{\gamma}(t, z, \lambda) & =\left(\frac{1}{i \lambda} \partial_{z}\right)^{\gamma} u(t, z, \lambda) .
\end{aligned}
$$

Il existe alors des constantes $A, B$ telles que pour tous $t \in I, \tau \in \mathbb{C} \backslash K_{1}$, $z \in \Omega$, on ait

$$
\begin{gathered}
\left|\partial_{\tau}^{l} e_{n}^{\alpha}\right| \leq A B^{n+l} n ! l ! \frac{1}{R_{0}^{|\alpha|}}(1+|\tau|)^{-2} \\
\left|q_{n, l, \alpha}^{\beta}\right| \leq A B^{n+l+|\alpha|} n ! \frac{1}{R_{0}^{|\beta|}} \\
\left|g_{n}^{\gamma}\right| \leq A B^{n} n ! \frac{1}{R_{0}^{|\gamma|}}(1+|\tau|)^{-2}
\end{gathered}
$$

De plus, d'après (3.15), pour $z \in \bar{\Omega}^{\prime},|\gamma| \leq \lambda / C_{0}$,

$$
\forall l, \quad \exists C_{l}^{\prime}, \quad\left|\partial_{t}^{l} u_{\gamma} e^{-\lambda \varphi(z)}\right| \leq C_{l}^{\prime} \lambda^{M+l} \widetilde{D}^{|\gamma|},
$$

pour $D<\widetilde{D}<R_{0}$. Soient $\psi \in C_{0}^{\infty}(\mathbb{R})$, telle que $\psi(r)=1$ pour $|r| \leq 1 / 2$, $\psi(r)=0$ pour $|r| \geq 1, \varepsilon_{0}>0$. Pour $\tilde{e} \in A^{-2}$, on définit $\operatorname{Op}_{\psi}(\tilde{e}) \operatorname{par}(3.12)$ en remplaçant $k(t, s)$ par $\psi\left((t-s) / \varepsilon_{0}\right) k(t, s)$. D'après $(3.8)$, on a

$$
\mathrm{Op}(\tilde{e})\left(0, C_{1}, C_{2}\right) v-\mathrm{Op}_{\psi}(\tilde{e})\left(0, C_{1}, C_{2}\right) v \in C^{\infty}\left(I, H_{\varphi-\varepsilon}\left(\Omega^{\prime}\right)\right)
$$

dès que $v \in C^{\infty}\left(I, H_{\varphi}\left(\Omega^{\prime \prime}\right)\right), \Omega^{\prime} \Subset \Omega^{\prime \prime} \Subset \Omega$, pourvu que $C_{1}, C_{2}$ soient assez grands. Pour prouver (3.26), on peut donc remplacer $\mathrm{Op}(e \circ q)$ et $\mathrm{Op}(e)$ par $\mathrm{Op}_{\psi}(e \circ q)$ et $\mathrm{Op}_{\psi}(e)$. On a, par définition,

$$
\mathrm{Op}_{\psi}(e) \mathrm{Op}(q) u(t, z, \lambda)=J
$$

$$
J=\sum_{E}(i \lambda)^{-n^{\prime}-n^{\prime \prime}} \int_{0}^{t_{0}} \psi\left(\frac{t-s}{\varepsilon_{0}}\right) k\left(e, 0 ; n^{\prime}, \alpha\right)(t, s)\left[q_{n^{\prime \prime}, 0,0}^{\beta} u_{\beta}\right]_{\alpha}(s) d s,
$$

où $E=\left\{n^{\prime} \leq \lambda / C_{1}, n^{\prime \prime} \leq \lambda / C_{1},|\alpha| \leq \lambda / C_{2},|\beta| \leq \lambda / C_{2}\right\}$. En choisissant $\varepsilon_{0}$ assez petit, on a alors

$$
J=\int_{0}^{t_{0}} \sum_{E^{\prime}} \mathscr{J}\left(n^{\prime}, n^{\prime \prime}, \alpha_{1}, \alpha_{2}, \beta, l\right),
$$




$$
\begin{aligned}
& \mathscr{J}\left(n^{\prime}, n^{\prime \prime}, \alpha_{1}, \alpha_{2}, \beta, l\right) \\
& =(i \lambda)^{-n^{\prime}-n^{\prime \prime}-\left|\alpha_{1}\right|} \psi\left(\frac{t-s}{\varepsilon_{0}}\right)(s-t)^{l} k\left(e, 0 ; n^{\prime}, \alpha_{1}+\alpha_{2}\right)(t, s) \\
& \quad \times \frac{\left(\alpha_{1}+\alpha_{2}\right) !}{\alpha_{2} !} q_{n^{\prime \prime}, l, \alpha_{1}}^{\beta}(t) u_{\beta+\alpha_{2}}(s), \\
& E^{\prime}=\left\{\left(n^{\prime}, n^{\prime \prime}, \alpha_{1}, \alpha_{2}, \beta, l\right),\left(n^{\prime}, n^{\prime \prime}, \alpha_{1}+\alpha_{2}, \beta\right) \in E, l \geq 0\right\} .
\end{aligned}
$$

Si $\mathscr{J}=\mathscr{J}^{+}+\mathscr{J}^{-}$est la décomposition associée à celle de $k$, on a

$$
\begin{aligned}
\mid \partial_{s, l^{\prime}}^{l_{0}} \mathcal{J}^{ \pm} & \left(n^{\prime}, n^{\prime \prime}, \alpha_{1}, \alpha_{2}, \beta, l\right) e^{-\lambda \varphi(z)} \mid \\
\leq & C_{l_{0}} \lambda^{M+l_{0}}\left(\frac{B n^{\prime}}{\lambda}\right)^{n^{\prime}}\left(\frac{B n^{\prime \prime}}{\lambda}\right)^{n^{\prime \prime}}\left(\varepsilon_{0} B\right)^{l} \\
& \times \alpha_{1} !\left(\frac{B}{\lambda R_{0}}\right)^{\left|\alpha_{1}\right|} \frac{\left(\alpha_{1}+\alpha_{2}\right) !}{\alpha_{1} ! \alpha_{2} !}\left(\frac{\widetilde{D}}{R_{0}}\right)^{|\beta|+\left|\alpha_{2}\right|} .
\end{aligned}
$$

Or, pour tout $\eta>0$, il existe $A_{\eta}, F_{\eta}$ tels que

$$
\forall \alpha_{1}, \alpha_{2}, \quad \frac{\left(\alpha_{1}+\alpha_{2}\right) !}{\alpha_{1} ! \alpha_{2} !} \leq A_{\eta} F_{\eta}^{\left|\alpha_{1}\right|}(1+\eta)^{\left|\alpha_{2}\right|} .
$$

En choisissant $\eta$ tel que $(1+\eta) \widetilde{D}<R_{0}$, il existe alors $\theta<1$ tel que pour tout $l_{0}$, il existe $C_{l_{0}}$ vérifiant

$$
\left|\partial_{s, l^{J^{\prime}}}^{l^{ \pm}}\left(n^{\prime}, n^{\prime \prime}, \alpha_{1}, \alpha_{2}, \beta, l\right) e^{-\lambda \varphi(z)}\right| \leq C_{l_{0}} \lambda^{M+l_{0}} \theta^{n^{\prime}+n^{\prime \prime}+l+\left|\alpha_{1}\right|+\left|\alpha_{2}\right|+|\beta|}
$$

dès que $n^{\prime}, n^{\prime \prime},\left|\alpha_{1}\right|,\left|\alpha_{2}\right|,|\beta| \leq \lambda / C, C$ assez grand, et pour tout $l$, en choisissant $\varepsilon_{0} B \leq \theta$. On peut donc, dans l'expression (3.37) de $J$, se restreindre à la somme sur

$$
E^{\prime \prime}=\left\{\left(n^{\prime}, n^{\prime \prime}, \alpha_{1}, \alpha_{2}, \beta, l\right), n^{\prime}+n^{\prime \prime}+\left|\alpha_{1}\right|+l \leq \lambda / C_{1},\left|\beta+\alpha_{2}\right| \leq \lambda / C_{2}\right\},
$$

avec $C_{1}$ et $C_{2}$ assez grands.

Si $\widetilde{J}=\int_{0}^{t_{0}} \sum_{E^{\prime \prime}} \mathcal{J}\left(n^{\prime}, n^{\prime \prime}, \alpha_{1}, \alpha_{2}, \beta, l\right)$, on a alors $J-\tilde{J} \in C^{\infty}\left(I, H_{\varphi-\varepsilon}\left(\Omega^{\prime}\right)\right)$ où $\varepsilon>0$ est indépendant de $u$. Or

$$
(s-t)^{l} k\left(e, 0 ; n^{\prime}, \alpha_{1}+\alpha_{2}\right)=(i \lambda)^{-l} k\left(\partial_{\tau}^{l} e, 0 ; n^{\prime}, \alpha_{1}+\alpha_{2}\right),
$$

ce qui conduit à

$$
\widetilde{J}=\mathrm{Op}_{\psi}(e \circ q)\left(0, C_{1}, C_{2}\right) u,
$$

d'où le lemme.

Soient $e \in A^{-2}(I, U, K), \Omega, \zeta_{0}, R_{0}$ tels que $\Omega \times B\left(\zeta_{0}, R_{0}\right) \subset U$. On a $\tau^{2} e \in A^{0}(I, U, K)$. Posons alors

$$
\left(\tau^{2} e_{n}\right)^{0}(t, z, \zeta, \lambda)=\lim _{\tau \rightarrow \infty} \tau^{2} e_{n}(t, \tau, z, \zeta, \lambda)
$$




$$
\begin{aligned}
& K^{ \pm}(t, s)\left(\zeta_{0}, C_{1}, C_{2}\right) \\
& \quad=\sum_{n \leq \lambda / C_{1}} \sum_{|\alpha| \leq \lambda / C_{2}}(i \lambda)^{-n} k^{ \pm}\left(e, \zeta_{0} ; n, \alpha\right)(t, s)\left(\frac{1}{i \lambda} \partial_{z}-\zeta_{0}\right)^{\alpha} .
\end{aligned}
$$

On notera que

$$
K^{+}(t, t)-K^{-}(t, t) \equiv 0
$$

$$
\begin{aligned}
& \frac{1}{\lambda^{2}}\left(\partial_{s} K^{+}(t, t)-\partial_{s} K^{-}(t, t)\right) \\
& \quad=\sum_{n \leq \lambda / C_{1}} \sum_{|\alpha| \leq \lambda / C_{2}}(i \lambda)^{-n} \frac{1}{\alpha !} \partial_{\zeta}^{\alpha}\left(\tau^{2} e_{n}\right)^{0}\left(t, z, \zeta_{0}, \lambda\right)\left(\frac{1}{i \lambda} \partial_{z}-\zeta_{0}\right)^{\alpha} .
\end{aligned}
$$

Par intégration par parties, on a alors le:

Lemme 3.4. Pour $u \in C^{\infty}\left(I, H_{\varphi}(\Omega)\right)$ on $a$

$$
\begin{aligned}
& \operatorname{Op}(e)\left(\zeta_{0}, C_{1}, C_{2}\right)\left[\left(\frac{1}{i \lambda} \partial_{t}\right)^{2} u\right] \\
& =\operatorname{Op}\left(\tau^{2} e\right)\left(\zeta_{0}, C_{1}, C_{2}\right)(u) \\
& +\frac{1}{\lambda^{2}}\left[K^{+}(t, 0) \partial_{t} u(0)-\partial_{s} K^{+}(t, 0) u(0)\right. \\
& \left.\quad-K^{-}\left(t, t_{0}\right) \partial_{t} u\left(t_{0}\right)+\partial_{s} K^{-}\left(t, t_{0}\right) u\left(t_{0}\right)\right]
\end{aligned}
$$

Remarque 3.1. Soient $\varphi=\varphi(x)$ une fonction réelle de classe $C^{2}$ près de $x_{0} \in$ $\mathbb{C}^{d}$ et

$$
P u(x, \lambda)=\left(\frac{\lambda}{2 \pi}\right)^{d} \iint_{\Sigma_{0}} e^{i \lambda(x-y) \xi} p(x, \xi, \lambda) u(y, \lambda) d y \wedge d \xi
$$

un opérateur pseudo-différentiel analytique au sens de Sjöstrand [S2], où $p$ est un symbole analytique défini près de $x_{0}, \xi_{0}=2 \partial_{x} \varphi\left(x_{0}\right) / i$ et où $\Sigma_{0}$ est un bon contour de la forme

$$
y=x_{0}+z, \quad \xi=\frac{2}{i} \frac{\partial \varphi}{\partial x}\left(x_{0}\right)-i C_{0} \bar{z}, \quad|z| \leq r,
$$

avec $r$ assez petit et $C_{0}$ assez grand. Alors, si on pose

$$
\operatorname{Op}(p)\left(\xi_{0}, C\right)(u)=\sum_{|\alpha| \leq \lambda / C} \frac{1}{\alpha !} \partial_{\xi}^{\alpha} p\left(x, \xi_{0}, \lambda\right)\left(\frac{1}{i \lambda} \partial_{x}-\xi_{0}\right)^{\alpha} u,
$$

on vérifie aisément que, si $\Omega$ est un voisinage de $x_{0}$ et $u \in H_{\varphi}(\Omega)$, il existe $\omega \Subset \Omega$ voisinage de $x_{0}$ et $C_{1}>0$ tels que $P u \in H_{\varphi}(\omega), \operatorname{Op}(p)\left(\xi_{0}, C\right)(u) \in$ $H_{\varphi}(\omega)$ pour $C \geq C_{1}$, et $P u-O p(p) u \in H_{\varphi-\varepsilon}(\omega)$, où $\varepsilon$ dépend de $C$, mais pas de $u$. Il n'y a donc pas de différence essentielle entre la présentation des opérateurs pseudo-différentiels analytiques donnée ici et celle de Sjöstrand [S2]. 
3.2. Régularité deux-microlocale pour le problème de Dirichlet. Soit $M=$ $\left\{(x, t) ; x \in \mathbb{R}^{n},|x|<a, 0 \leq t<1\right\}$, que l'on considère comme une variété analytique à bord $\partial M=M \cap\{t=0\}$, et soit $P=D_{t}^{2}+R\left(t, x, D_{x}\right)$ un opérateur différentiel d'ordre 2 à coefficients analytiques sur $M$, à symbole principal $p=\tau^{2}+r(t, x, \xi)$ réel. On pose $T^{*} M=T^{*} \partial M \cup T^{*} \stackrel{\circ}{M}$, muni de la topologie naturelle. On pose $r_{0}(x, \xi)=r(0, x, \xi)$, et on suppose que $\partial r_{0} / \partial \xi(x, \xi) \neq 0$ pour $\xi \neq 0$. Les régions elliptique $\mathscr{E}$, glancing $\mathscr{G}$, et hyperbolique $\mathscr{H}$ de $T^{*} \partial M \backslash \partial M=\dot{T}^{*} \partial M$ sont définies respectivement par $r_{0}>0$, $r_{0}=0$, et $r_{0}<0$.

Soient $\rho_{0}=\left(x_{0}, \xi_{0}\right) \in \dot{T}^{*} \partial M$ et $s$ une fonction analytique réelle définie près de $\rho_{0}$, homogène en $\xi$, et vérifiant

$$
s\left(\rho_{0}\right)=0 ; \quad d s\left(\rho_{0}\right) \wedge \xi_{0} d x \neq 0 ; \quad\left\{r_{0}, s\right\}\left(\rho_{0}\right) \neq 0 .
$$

On note $V=s^{-1}(0)$. C'est une variété involutive régulière de codimension 1 dans $\dot{T}^{*} \partial M$, transverse en $\rho_{0}$ au champ hamiltonien $H_{r_{0}}$.

Si $f \in \mathscr{D}^{\prime}(\partial M)$, on notera $S S_{V}^{2,1}(f)$ le deuxième micro-support analytique à croissance de $f$ le long de $V$. (Cf. [L1].) C'est un fermé conique du fibré normal $T_{V}\left(\dot{T}^{*} \partial M\right)$ à $V$ dans $\dot{T}^{*} \partial M$, orienté ici de sorte que $\left(\rho_{0}, \pm 1\right) \notin$ $S S_{V}^{2,1}(f)$ entraîne $S S(f) \cap\{ \pm s>0\}=\varnothing$ au voisinage de $\rho_{0}$.

Soit $f=f(t, x)$ une distribution prolongeable sur $\stackrel{\circ}{M}$ vérifiant

$$
P f=0 \text { dans } \stackrel{\circ}{M} \text {. }
$$

Définition 3.4. On dit que $f$ est $V$-sortante en $\rho_{0}$ si et seulement si

(i) Si $\rho_{0} \in \mathscr{E}$, pas de condition.

(ii) $\mathrm{Si} \rho_{0} \in \mathscr{H}$ et si $\gamma_{ \pm}$sont les deux demi-bicaractéristiques de $p$ passant au-dessus de $\rho_{0}$ et contenues dans $T^{*} \stackrel{\circ}{M}$, on a $\gamma_{+} \cap S S(f)=\varnothing$ ou $\gamma_{-} \cap S S(f)=$ $\varnothing$.

(iii) Si $\rho_{0} \in \mathscr{G}$, il existe $\varepsilon>0$ tel que $0 \leq t \leq \varepsilon,\left|\rho-\rho_{0}\right| \leq \varepsilon$, et $s(\rho)<0$ entraînent, pour tout $\tau,(\rho, t, \tau) \notin S S_{b}(f)$, où $S S_{b}$ désigne le front d'onde analytique au bord (cf. [S1]).

Remarque 3.2. L'opérateur $P$ étant à caractéristiques simples réelles, on peut en utilisant le théorème de propagation des singularités à l'intérieur, remplacer (iii) par

(iii) $^{\prime}$ Il existe $\varepsilon>0$ tel que $\left|\rho-\rho_{0}\right| \leq \varepsilon, s(\rho)<0$, et $0 \leq t \leq C$ dist $\left(\rho, s^{-1}(0)\right)$ entraînent, pour tout $\tau,(\rho, t, \tau) \notin S S_{b}(f)$ (avec $C>0$ donné). Cette dernière condition est invariante par changement de coordonnées.

Remarque 3.3. On notera que, si $f$ est $V$-sortante en un point $\rho_{0} \in \mathscr{G}$, alors $f$ en particulier $V$-sortante en tout point $\rho \in \mathscr{H} \cap V$ dans un voisinage de $\rho_{0}$.

Théorème 3.1. Soit $f$ une distribution prolongeable sur $M$ vérifiant $P f=0$ dans $\stackrel{\circ}{M}, V$-sortante en $\rho_{0}$. On suppose que $\left(\rho_{0},-1\right) \notin S S_{V}^{2,1}\left(\left.f\right|_{t=0}\right)$. Alors

$$
\left(\rho_{0},-1\right) \notin S S_{V}^{2,1}\left(\left.\frac{\partial f}{\partial t}\right|_{t=0}\right) \text {. }
$$


Remarque 3.4. Si $f$ vérifie la condition de Dirichlet $\left.f\right|_{t=0}=0$, on a $S S_{b}(f) \cap$ $\dot{T}^{*} \partial M \subset \mathscr{G} \cup \mathscr{H}$. Si $\rho_{0} \in \mathscr{G}$, la bicaractéristique de $V$ passant par $\rho_{0}$ est transverse à $\mathscr{G}$ d'après $(3.49)$, donc rentre dans $\mathscr{E}$. Par le principe du maximum (cf. [L2]), $\left(\rho_{0},-1\right) \notin S S_{V}^{2,1}\left(\partial f /\left.\partial t\right|_{t=0}\right)$ entraîne $\rho_{0} \notin S S_{b}(f)$, et l'on retrouve un théorème micro-hyperbolique au bord de J. Sjöstrand [S3].

Démonstration $d u$ théorème. Si $\rho_{0} \in \mathscr{E} \cup \mathscr{H}$, l'hypothèse $f \quad V$-sortante assure l'existence d'un opérateur pseudo-différentiel analytique d'ordre $1 E$ tel que

$$
\rho_{0} \notin S S\left(\left.\frac{\partial f}{\partial t}\right|_{t=0}-E\left(\left.f\right|_{t=0}\right)\right),
$$

et le théorème est alors conséquence du fait que les opérateurs pseudo-différentiels sont deux-microlocaux. On peut donc supposer $\rho_{0} \in \mathscr{G}$.

Par (iii) et la propagation des singularités à l'intérieur, on sait que

$$
0<t \leq \varepsilon / 2, \quad\left|\rho-\rho_{0}\right| \leq \varepsilon / 2, \quad s(\rho) \leq 0 \quad \Rightarrow \quad(\rho, t, \tau) \notin S S(f)
$$

si $\varepsilon$ est assez petit.

Soit $\chi:\left(T^{*} \mathbb{C}^{d}, \rho_{0}\right) \rightarrow\left(T^{*} \mathbb{C}^{d},\left(z_{0}, \zeta_{0}\right)=(0,0)\right)$ une transformation canonique complexe locale telle que $\chi\left(T^{*} \mathbb{R}^{d}\right)=\Lambda_{\varphi_{0}}$, où

$$
\begin{aligned}
\varphi_{0}(z) & =\frac{1}{2}\left(\operatorname{Re} z_{1}\right)^{2}+\frac{1}{2}\left(\operatorname{Im} z^{\prime}\right)^{2}, \\
\Lambda_{\varphi_{0}} & =\left\{\left(z, \frac{2}{i} \frac{\partial \varphi_{0}}{\partial z}\right)\right\}=\left\{\left(z_{1}, z^{\prime},-i \operatorname{Re} z_{1},-\operatorname{Im} z^{\prime}\right)\right\},
\end{aligned}
$$

et telle que, en notant $\nu$ le signe de $\left\{r_{0}, s\right\}\left(\rho_{0}\right)$,

$$
\begin{aligned}
\nu R_{0}(z, \zeta) & =r_{0} \circ \chi^{-1}(z, \zeta)=\zeta_{1}+i z_{1}, \\
S(z, \zeta) & =s \circ \chi^{-1}(z, \zeta)=i \zeta_{1} l(z, \zeta),\left.l\right|_{\Lambda_{\varphi_{0}}}>0 .
\end{aligned}
$$

Soit aussi $T$ une transformation de Fourier-Bros-Iagolnitzer ([S2]) associée à $\chi$,

$$
T f(z, \lambda)=\int e^{i \lambda g(z, x)} f(x) \psi(x) d x
$$

où le graphe de $\chi$ est donné par $\left\{\left(x,-g_{x}^{\prime} ; z, g_{z}^{\prime}\right)\right\}, g\left(0, x_{0}\right)=0$, det $g_{z x}^{\prime \prime}\left(0, x_{0}\right)$ $\neq 0$, et où $\psi \in C_{0}^{\infty}$ est supportée dans un voisinage de $x_{0}$ et vaut 1 près de $x_{0}$. Posons

$$
u(t, z, \lambda)=T(f(t, \cdot))(z, \lambda) .
$$

Soit $\left.t_{0} \in\right] 0,1[$. Puisque $f$ est solution prolongeable de $P f=0$, on a

$$
\exists \nu_{0} \in \mathbb{R}, \quad \forall k \geq 0, \quad f \in C^{k}\left(\left[0, t_{0}\right], H^{\nu_{0}-k}(|x|<a / 2)\right),
$$

et il existe donc un voisinage $\Omega_{0}$ de $z_{0}=0$ tel que

$$
u \in C^{\infty}\left(\left[0, t_{0}\right] ; H_{\varphi_{0}}\left(\Omega_{0}\right)\right) .
$$


De plus, il existe un symbole analytique $q$ défini près de $t \in\left[0, t_{0}\right],\left(z_{0}, \zeta_{0}\right)=$ $(0,0)$,

(3.60) $q(t, z, \zeta, \lambda)=\sum_{n \geq 0}(i \lambda)^{-n} q_{n}(t, z, \zeta), \quad q_{0}(t, \chi(x, \xi))=r(t, x, \xi)$,

tel que, pour $C_{1}, C_{2}$ assez grands, on ait

$$
\begin{aligned}
& \lambda^{2} \mathrm{Op}(q)\left(0, C_{1}, C_{2}\right) e^{i \lambda g(z, x)} \\
& \quad={ }^{t} R\left(t, x, D_{x}\right)\left(e^{i \lambda g(z, x)}\right)+e^{i \lambda g(z, x)} b(t, x, z, \lambda),
\end{aligned}
$$

où $b$ est un symbole analytique près de $t \in\left[0, t_{0}\right], x=x_{0}, z=0$ avec $b$ dominé par $e^{-\varepsilon \lambda}$ pour un $\varepsilon>0$. Si $\tilde{p}=\tau^{2}+q$, on a alors

$$
\mathrm{Op}(\tilde{p})\left(0, C_{1}, C_{2}\right) u \in C^{\infty}\left(\left[0, t_{0}\right] ; H_{\varphi_{0}-\varepsilon_{0}}\left(\Omega_{1}\right)\right), \quad \varepsilon_{0}>0 .
$$

Ici $\Omega_{1} \Subset \Omega_{0}$ et $C_{1}, C_{2}$ sont compatibles avec les notations du Lemme 3.1. Pour $z=\left(z_{1}, z^{\prime}\right) \in \mathbb{C}^{d}$, on pose

$$
\begin{aligned}
x_{1} & =\operatorname{Re} z_{1}, \quad y=\left(\operatorname{Im} z_{1}, z^{\prime}\right), \\
\varphi_{-}(z) & =\frac{1}{2}\left(\operatorname{Im} z^{\prime}\right)^{2}+\frac{1}{2}\left[\left(\operatorname{Re} z_{1}\right)_{+}\right]^{2},
\end{aligned}
$$

où $x_{+}$désigne $\max (x, 0)$. Par hypothèse on a $\left(\rho_{0},-1\right) \notin S S_{V}^{2,1}\left(\left.f\right|_{t=0}\right)$ et on peut donc supposer, si $\Omega_{0}$ est assez petit,

$$
\forall z \in \Omega_{0}, \quad|u(0, z, \lambda)| \leq A \lambda^{M} e^{\lambda \varphi_{-}(z)} .
$$

On fixe à présent $d_{1}>0, t_{0}>0$ petits tels que

$$
\|z\| \leq 2 d_{1} \Rightarrow z \in \Omega_{1},
$$

(3.67) $\|z\| \leq 2 d_{1}, \quad \operatorname{Re} z_{1}<0 \Rightarrow u \in C^{\infty}\left(\left[0, t_{0}\right] ; H_{\varphi_{0}-\varepsilon}\right)$ près de $z$

$\|z\| \leq 2 d_{1}, \quad \operatorname{Re} z_{1} \leq 0, \quad t_{0}^{\prime}>0 \Rightarrow u \in C^{\infty}\left(\left[t_{0}^{\prime}, t_{0}\right] ; H_{\varphi_{0}-\varepsilon}\right)$ près de $z$,

ce qui est possible d'après (3.52).

Soit $\alpha>0$ à fixer ultérieurement. Il existe trois réels $-d_{1}<s_{3}(\alpha)<s_{2}(\alpha)<$ $s_{1}(\alpha)<0$ et une fonction $\theta_{\alpha}=\theta_{\alpha}(s) \in C^{\infty}\left(\left[-d_{1},+\infty[)\right.\right.$ tels que

$$
\begin{aligned}
\sup \left(0, \frac{s^{2}}{2}-\alpha\right) & \leq \theta_{\alpha}(s) \leq \frac{s^{2}}{2} ; \quad s+\alpha \geq \theta_{\alpha}^{\prime}(s) \geq s, \\
\theta_{\alpha}(s) & =\frac{s^{2}}{2} \quad \text { si } s \in\left[s_{1}(\alpha), \infty[\right. \\
\theta_{\alpha}^{\prime}(s)>s & \text { si } s \in\left[-d_{1}, s_{1}(\alpha)[\right. \\
\theta_{\alpha}(s) \equiv 0 & \text { si } s \in\left[s_{3}(\alpha), s_{2}(\alpha)\right] .
\end{aligned}
$$

Soit aussi $\phi=\phi(y) \in C^{\infty}$, positive, ne dépendant que de

$$
|y|=\left(\left(\operatorname{Im} z_{1}\right)^{2}+\left|z_{2}\right|^{2}+\cdots+\left|z_{d}\right|^{2}\right)^{1 / 2},
$$


croissante en $|y|$, nulle pour $|y| \leq d_{1} / 4$, telle que $\phi\left(d_{1}\right)>d_{1}$. Pour $\left|\operatorname{Re} z_{1}\right| \leq$ $d_{1},|y| \leq d_{1}$, posons

$$
\varphi(z)=\varphi_{0}(z)+\theta_{\alpha}\left(\operatorname{Re} z_{1}+\phi(y)\right)-\frac{1}{2}\left(\operatorname{Re} z_{1}+\phi(y)\right)^{2} .
$$

On a

$$
\begin{aligned}
\frac{2}{i} \frac{\partial \varphi}{\partial z^{\prime}} & =-\operatorname{Im} z^{\prime}+\left[\theta_{\alpha}^{\prime}\left(\operatorname{Re} z_{1}+\phi(y)\right)-\left(\operatorname{Re} z_{1}+\phi(y)\right)\right] \frac{2}{i} \frac{\partial \phi}{\partial z^{\prime}} \\
& =\zeta^{\prime} \\
\frac{2}{i} \frac{\partial \varphi}{\partial z_{1}} & =-i \operatorname{Re} z_{1} \\
& +\left[\theta_{\alpha}^{\prime}\left(\operatorname{Re} z_{1}+\phi(y)\right)-\left(\operatorname{Re} z_{1}+\phi(y)\right)\right] \frac{1}{i}\left(1-i \frac{\partial \phi}{\partial y_{1}}\right) \\
= & \zeta_{1},
\end{aligned}
$$

et

$$
\varphi(z) \leq \varphi_{0}(z) ; \quad \varphi(z)=\varphi_{0}(z) \text { si } \operatorname{Re} z_{1}+\phi(y) \geq s_{1}(\alpha) .
$$

Pour $\delta \in[0,1]$, on pose

$$
\varphi_{\delta}(z)=\delta \varphi(z)+(1-\delta) \varphi_{0}(z) .
$$

On a

$$
\operatorname{Im}\left(\zeta_{1}+i z_{1}\right)=-\theta_{\alpha}^{\prime}\left(\operatorname{Re} z_{1}+\phi(y)\right)+\left(\operatorname{Re} z_{1}+\phi(y)\right) \leq 0,
$$

et, si l'on pose $|\nabla \phi|_{\infty}=\sup \left\{|\nabla \phi(y)|,|y| \leq d_{1}\right\}$,

$$
\left|\frac{2}{i} \frac{\partial \varphi_{\delta}}{\partial z}(z)-\frac{2}{i} \frac{\partial \varphi_{0}}{\partial z}(z)\right| \leq \operatorname{Cte} \delta\left(1+|\nabla \phi|_{\infty}\right)\left|\operatorname{Im}\left(\zeta_{1}+i z_{1}\right)\right|,
$$

où la constante est indépendante de $\alpha$. Si $\Omega_{2}=\left\{\left|\operatorname{Re} z_{1}\right|<d_{1},|y|<d_{1}\right\} \Subset \Omega_{1}$, on a donc en tenant compte de (3.54),

(3.79) $\nu \operatorname{Im}\left(q_{0}\left(t, z, \frac{2}{i} \frac{\partial \varphi_{\delta}}{\partial z}(z)\right)\right) \leq \delta \operatorname{Im}\left(\zeta_{1}+i z_{1}\right)\left[1-\operatorname{Cte} t\left(1+|\nabla \phi|_{\infty}\right)\right]$,

où la constante est indépendante de $\alpha$. Soit $\left.t_{1} \in\right] 0, t_{0}[$ tel que

$$
1-\operatorname{Cte} t_{1}\left(1+|\nabla \phi|_{\infty}\right) \geq \frac{1}{2} \text {. }
$$

D'après (3.67), (3.68), et (3.75), il existe $\alpha_{0}>0$ et $\beta>0$ tels que, pour tout $\alpha \in] 0, \alpha_{0}[$,

$$
\begin{aligned}
& u \in C^{\infty}\left(\left[\frac{t_{1}}{2}, t_{1}\right] ; H_{\varphi}\left(\Omega_{2}\right)\right), \\
& u \in C^{\infty}\left(\left[0, t_{1}\right] ; H_{\varphi}\left(\Omega_{2} \cap\left\{\operatorname{Re} z_{1}<-d_{1} / 2\right\}\right)\right), \\
& u \in C^{\infty}\left(\left[0, t_{1}\right] ; H_{\varphi}\left(\Omega_{2} \cap\left\{|y| \geq d_{1}-\beta\right\}\right)\right) .
\end{aligned}
$$

On pose $\Omega_{3}=\left\{\left|\operatorname{Re} z_{1}\right|<5 d_{1} / 6,|y|<d_{1}-\beta / 3\right\} \Subset \Omega_{2}$. D'après le Lemme 3.1 et (3.62), si $C_{1}^{\prime}$ et $C_{2}^{\prime}$ sont assez grands, il existe $\varepsilon_{1}>0$ tel que

$$
\mathrm{Op}(\tilde{p})\left(0, C_{1}^{\prime}, C_{2}^{\prime}\right) u \in C^{\infty}\left(\left[0, t_{1}\right] ; H_{\varphi_{0}-\varepsilon_{1}}\left(\Omega_{3}\right)\right) \text {. }
$$


On fixe à présent $\alpha \in] 0, \inf \left(\alpha_{0}, \varepsilon_{1} / 2\right)$ [ et on va prouver que, pour tout $\delta \in$ $[0,1]$, on a

$$
u \in C^{\infty}\left(\left[0, t_{1}\right] ; H_{\varphi_{\delta}}\left(\Omega_{2}\right)\right)
$$

D'après (3.67), (3.75), il existe $\beta^{\prime}>0, s_{1}\left(\alpha^{\prime}\right)-\beta^{\prime}>s_{2}\left(\alpha^{\prime}\right)$, tel que

$$
u \in C^{\infty}\left(\left[0, t_{1}\right] ; H_{\varphi}\left(\Omega_{2} \cap\left\{\operatorname{Re} z_{1}>s_{1}(\alpha)-\beta^{\prime}\right\}\right)\right)
$$

et pour $\delta$ assez petit, (3.84) résulte de (3.67).

Soit $\left.\left.\delta_{0} \in\right] 0,1\right]$ et supposons que $u$ vérifie $(3.84)$ pour tout $\delta<\delta_{0}$. Soit $z^{0}=\left(z_{1}^{0}, y^{0}\right) \in \Omega_{2}$, avec $-3 d_{1} / 4 \leq \operatorname{Re} z_{1}^{0} \leq s_{1}(\alpha)-\beta^{\prime} / 2,|y| \leq d_{1}-\beta / 2$. Soit $\zeta_{0}=\left(2 \partial \varphi_{\delta} / i \partial z\right)\left(z^{0}\right)$. D'après $(3.79),(3.77)$, et (3.71), il existe un voisinage $U$ de $\left(z^{0}, \zeta^{0}\right)$ et $c>0$ tels que

$\forall \tau \in \mathbb{R}, \quad \forall t \in\left[0, t_{1}\right], \quad \forall(z, \zeta) \in \bar{U}, \quad\left|\tau^{2}+q_{0}(t, z, \zeta)\right| \geq c(1+|\tau|)^{2}$,

et les racines en $\tau \in \mathbb{C}$ de l'équation $\tau^{2}+q_{0}=0$ restent dans un compact $K$ de $\mathbb{C} \backslash \mathbb{R}$. Soit

$$
e=(\tilde{p})^{-1} \in A^{-2}\left(\left[0, t_{1}\right], U, K\right)
$$

l'inverse pseudo-différentiel de $\tilde{p}$; soient $\omega_{1} \Subset \omega_{0}$ deux petits voisinages de $z^{0}$ et $R_{0}>0$ tels que

$$
\omega_{0} \times B\left(\zeta^{0}, R_{0}\right) \subset U, \quad \sup _{z \in \omega_{0}}\left\|\frac{2}{i} \frac{\partial \varphi_{\delta_{0}}}{\partial z}(z)-\zeta^{0}\right\|<D_{0}<R_{0} .
$$

Comme on peut toujours supposer que l'on a

$$
\sup _{z \in \Omega_{2}, \delta \in[0,1]}\left\|\frac{2}{i} \frac{\partial \varphi_{\delta}}{\partial z}(z)\right\|<D<R,
$$

où $R$ est un rayon de convergence pour $\tilde{p}$ en $\zeta=0$, on peut supposer dans (3.83) que $C_{1}^{\prime} \geq 2 B / e, C_{2}^{\prime} \geq\left(D \operatorname{dist}\left(\bar{\Omega}_{3}, \Omega_{2}^{c}\right)\right)^{-1}$, où $B$ est la constante intervenant dans $\tilde{p}$ pour des estimations du type (3.2).

On a alors

$$
\operatorname{Op}(\tilde{p})\left(0, C_{1}^{\prime}, C_{2}^{\prime}\right) u \in C^{\infty}\left(\left[0, t_{1}\right] ; H_{\varphi_{\delta_{0}}-\varepsilon_{1} / 2}\left(\Omega_{3}\right)\right) .
$$

D'après le Lemme 3.1 , si $C_{2}^{\prime \prime}=C_{2}^{\prime}+\left(D_{0} \operatorname{dist}\left(\bar{\omega}_{1}, \omega_{0}^{c}\right)\right)^{-1}+\left(D \operatorname{dist}\left(\bar{\omega}_{1}, \omega_{0}^{c}\right)\right)^{-1}$, il existe $\varepsilon>0$ tel que

$$
\operatorname{Op}(\tilde{p})\left(0, C_{1}^{\prime}, C_{2}^{\prime \prime}\right) u \in C^{\infty}\left(\left[0, t_{1}\right] ; H_{\varphi_{\delta_{0}}-\varepsilon}\left(\Omega_{3}\right)\right),
$$

et, d'après le Lemme 3.2, il existe alors $\varepsilon>0$ tel que

$$
\mathrm{Op}(\tilde{p})\left(\zeta_{0}, C_{1}^{\prime}, C_{2}^{\prime \prime}\right) u \in C^{\infty}\left(\left[0, t_{1}\right] ; H_{\varphi_{\delta_{0}}-\varepsilon}\left(\omega_{1}\right)\right)
$$


Toujours d'après le Lemme 3.1, (3.90) reste vraie, en diminuant éventuellement $\varepsilon$, pour tous $\widetilde{C}_{1} \geq C_{1}^{\prime}, \widetilde{C}_{2} \geq C_{2}^{\prime \prime}$. Si $\widetilde{C}_{1}$ et $\widetilde{C}_{2}$ sont assez grandes, on peut alors appliquer les Lemmes 3.3 et 3.4. On obtient

$$
\begin{aligned}
\operatorname{Op}(1) & u-\operatorname{Op}(e) \operatorname{Op}(\tilde{p})\left(\zeta_{0}, \widetilde{C}_{1}, \widetilde{C}_{2}\right) u \\
+ & \frac{1}{\lambda^{2}}\left[K^{+}(t, 0) \partial_{t} u(0)-\partial_{s} K^{+}(t, 0) u(0)\right. \\
& \left.\quad-K^{-}\left(t, t_{1}\right) \partial_{t} u\left(t_{1}\right)+\partial_{s} K^{-}\left(t, t_{1}\right) u\left(t_{1}\right)\right] \\
\in & C^{\infty}\left(\left[0, t_{1}\right] ; H_{\varphi_{\delta_{0}}-\varepsilon}\left(\omega_{1}\right)\right) .
\end{aligned}
$$

En faisant $t=0$ et en utilisant (3.90) et (3.8), on en déduit, pour $z \in \omega_{2} \Subset \omega_{1}$,

$$
K^{+}(0,0) \partial_{t} u(0)=\partial_{s} K^{+}(0,0) u(0)-\lambda^{2} u(0)+w
$$

où $w \in H_{\varphi_{\delta_{0}}-\varepsilon}\left(\omega_{2}\right)$. Or $K^{+}(0,0)$ et $\partial_{s} K^{+}(0,0)$ sont des opérateurs pseudodifférentiels elliptiques sur $U$ de degrés 1 et 2 . Si $\delta_{0}<1$, on déduit de (3.65) et (3.92), $\partial_{t} u(0) \in H_{\varphi_{\delta_{0}}-\varepsilon}\left(\omega_{3}\right)$ si $\omega_{3} \Subset \omega_{2}$, d'où, puisque $\mathrm{Op}(1) u=u$, et d'après (3.91) et (3.80),

$$
u \in C^{\infty}\left(\left[0, t_{1}\right] ; H_{\varphi_{\delta_{0}}-\varepsilon}\left(\omega_{3}\right)\right),
$$

d'où (3.84) en utilisant (3.71), (3.72), et (3.87), pour tout $\delta \in] 0,1[$. On a alors encore (3.91) et (3.92) pour $\delta_{0}=1$, d'où il résulte, d'après (3.65), puisque $\varphi=\varphi_{1} \geq \varphi_{-}$,

$$
\left|\partial_{t} u(0, z, \lambda)\right| \leq A^{\prime} \lambda^{M+1} e^{\lambda \varphi(z)}
$$

pour $z=\left(z_{1}, z^{\prime}\right), \operatorname{Re} z_{1} \in\left[s_{3}(\alpha), s_{2}(\alpha)\right],|y| \leq d_{1} / 4$.

On a $\left.\nu r_{0} \circ \chi^{-1}\right|_{\Lambda_{\varphi_{0}}}=-\operatorname{Im} z_{1}$; comme le théorème est déjà démontré aux points elliptiques et hyperboliques, pour tout $z_{0}=\left(z_{1,0}, z_{0}^{\prime}\right)$ près de 0 avec $\operatorname{Im} z_{1,0} \neq 0$, il existe un voisinage $V_{0}$ de $z_{0}$ tel que

$$
\forall z \in V_{0}, \quad\left|\partial_{t} u(0, z, \lambda)\right| \leq \operatorname{Cte} \lambda^{M+1} e^{\lambda \varphi_{-}(z)} .
$$

On a $\varphi(z)=\varphi_{-}(z)=\left(\operatorname{Im} z^{\prime}\right)^{2} / 2$ pour $|y| \leq d_{1} / 4, \operatorname{Re} z_{1} \in\left[s_{3}(\alpha), s_{2}(\alpha)\right]$. Si $\alpha$ est assez petit, (3.94), (3.95), et le principe du maximum entraînent au voisinage de $z=0$

$$
\left|\partial_{t} u(0, z, \lambda)\right| \leq \operatorname{Cte} \lambda^{M+1} e^{\lambda \varphi_{-}(z)},
$$

ce qui achève la démonstration du théorème.

Remarque 3.5. L'identité (3.92) fournit une relation pseudo-différentielle entre les traces dans $\operatorname{Re} z_{1}<0$.

3.3. Changement d'inconnues et écriture du système. Introduisons tout d'abord les ondes réfléchies sur $\Delta_{ \pm}$à partir de la solution $u_{i}$ donnée par (1.6). 
Soient $\theta_{ \pm}$les fonctions analytiques définies dans un voisinage de $x=y=0$ par

$$
\begin{gathered}
\left(\partial_{x} \theta_{ \pm}\right)^{2}+\left(\partial_{y} \theta_{ \pm}\right)^{2}=1, \quad \theta_{ \pm}-\left.\theta_{i}\right|_{\widetilde{\Delta}_{ \pm}}=0, \\
\partial_{ \pm} \theta_{ \pm}(0,0)=-\partial_{ \pm} \theta_{i}(0,0) .
\end{gathered}
$$

Alors il existe des symboles analytiques $\sigma_{ \pm}$près de $t=x=y=0$ tels que les fonctions données par

$$
u_{r, \pm}(t, x, y)=\frac{1}{2 \pi} \int_{-\infty}^{\infty} e^{i \tau\left(t-\theta_{ \pm}(x, y)\right)} \sigma_{ \pm}(x, y, \tau) d \tau
$$

soient solutions de $\square u_{r, \pm}=0$ (modulo une fonction analytique réelle) et vérifient

$$
\left.u_{r, \pm}\right|_{\widetilde{\Delta}_{ \pm}}=\left.u_{i}\right|_{\widetilde{\Delta}_{ \pm}}
$$

Les symboles $\sigma_{ \pm}$sont entièrement caractérisés par les équations de transport classiques (voir par exemple [H, Théorème 24.2.3]). On a en particulier

$$
\sigma_{ \pm}\left|\tilde{\Delta}_{ \pm}=\sigma_{i}\right|_{\tilde{\Delta}_{ \pm}}
$$

Posons alors

$$
\begin{aligned}
& u_{ \pm}=u_{i}-u_{r, \pm}, \\
& \tilde{f}_{ \pm}=v_{ \pm}-1_{x>0} \partial_{ \pm} u_{ \pm} .
\end{aligned}
$$

$\operatorname{De}(3.100)$ et de la première équation de transport, on déduit que $u_{ \pm} \in H_{\mathrm{loc}}^{1}\left(\mathbb{R}^{3}\right)$, $\tilde{f}_{ \pm} \in L_{\text {loc }}^{2}\left(\Delta_{ \pm}\right)$, et $\sigma_{ \pm}$a les mêmes propriétés d'holomorphie en $\tau$ que $\sigma_{i}$, donc

$$
\operatorname{supp}\left(\tilde{f}_{ \pm}\right) \subset\{0 \leq x \leq C t\} .
$$

On note $D_{+}=\{(t, x, y), y>a(x)\}, D_{-}=\{(t, x, y), y<b(x)\}$. On introduit les sous-variétés involutives $V_{ \pm}=\{x=0\}$ de $T^{*} \widetilde{\Delta}_{ \pm}$, dont on oriente les fibrés normaux comme à la $\S 3.2$, en choisissant $s(x)=x$. On dira qu'une solution $f$ de $\square f=0$ dans $D_{ \pm}$est $V_{ \pm}$-entrante si elle est $\left(-V_{ \pm}\right)$-sortante, où $-V_{ \pm}$est $V_{ \pm}$avec l'orientation opposée.

Proposition 3.1. Pour tout $\varepsilon_{0}>0$, il existe $\varepsilon_{1}>0$ tel que

$$
S S\left(\tilde{f}_{ \pm}\right) \cap\left\{x>0, t \leq \varepsilon_{1}\right\} \subset\left|\tilde{\xi}+\frac{\tau}{\cos \gamma_{ \pm}}\right|<\varepsilon_{0} .
$$

Si $\rho=(x=0, t, \tilde{\xi}, \tau), t \leq \varepsilon$, on $a$

$$
(\rho,+1) \notin S S_{V_{ \pm}}^{2.1}\left(\tilde{f}_{ \pm}\right) \text {pour }\left|\tilde{\xi}+\frac{\tau}{\cos \gamma_{ \pm}}\right| \geq \varepsilon_{0} .
$$

Démonstration. On remarque que $\left.\tilde{f}_{ \pm}\right|_{x>0}=\left.\partial_{ \pm}\left(u-u_{ \pm}\right)\right|_{x>0}$, et que $\square\left(u-u_{ \pm}\right)$ est analytique jusqu'au bord dans $D_{ \pm}$, avec, par construction,

$$
\begin{aligned}
& u-\left.u_{ \pm}\right|_{\partial D_{ \pm} \cap\{x>0\}}=0, \\
& u-\left.u_{ \pm}\right|_{t<0}=\left.u_{r . \pm}\right|_{t<0} .
\end{aligned}
$$


On notera que, si $g_{1}, g_{2} \in \mathscr{D}^{\prime}\left(\widetilde{\Delta}_{ \pm}\right)$sont telles que $\left.g_{1}\right|_{x>0}=\left.g_{2}\right|_{x>0}$, alors, pour tout $\rho \in V_{ \pm}$, on a $(\rho,+1) \notin S S_{V_{ \pm}}^{2,1}\left(g_{1}-g_{2}\right)$. Compte tenu de (3.106) et du Théorème $3.1,(3.105)$ sera donc prouvé si nous montrons que $u-u_{ \pm}$est $V_{ \pm}$-entrante au point $\rho$. (On vérifie en effet aisément que $V_{ \pm}$satisfait aux conditions de transversalité requises pour appliquer le Théorème 3.1.) Nous allons prouver cette propriété de $u-u_{ \pm}$en même temps que (3.104).

Soit $\varepsilon_{0}>0$, et soit $\rho=(x, t, \tilde{\xi}, \tau)$, avec $0 \leq x \leq c t,\left|\tilde{\xi}+\tau / \cos \gamma_{ \pm}\right| \geq \varepsilon_{0}$, hyperbolique ou glancing. (Le cas elliptique est sans objet pour (3.105) et, pour (3.104), est conséquence de (3.106) et de la régularité elliptique microlocale.) Supposons d'abord de plus $\left|\tilde{\xi}-\tau / \cos \gamma_{ \pm}\right| \geq \varepsilon_{0}^{\prime}$. Alors il existe $\varepsilon_{1}>0$ tel que, si $t \leq \varepsilon_{1}$, la demi-bicaractéristique $\gamma$ issue de $\rho$, contenue dans $D_{ \pm}$ et dirigée vers $t<0$ ne recoupe pas $\widetilde{\Delta}_{ \pm}$avant d'entrer dans $t<0$. Par définition, la distance à $\widetilde{\Delta}_{ \pm}$est décroissante avec $t$ le long de $\gamma$ près de $\widetilde{\Delta}_{ \pm}$. Or $u_{r, \pm}$ a été construite pour que, sur les bicaractéristiques contenues dans $S S\left(u_{r, \pm}\right) \cap D_{ \pm}$, la distance à $\widetilde{\Delta}_{ \pm}$soit croissante avec $t$ près de $\widetilde{\Delta}_{ \pm}$. La solution $u_{r, \pm}$ est donc régulière le long de $\gamma$, et, par le théorème de propagation des singularités et (3.107), on conclut que $\gamma \cap S S\left(u-u_{ \pm}\right)=\varnothing$. Si $x=0$, cela signifie exactement que $u-u_{ \pm}$est $V_{ \pm}$-entrante. Si $x>0$, la condition (3.106) et le théorème de réflexion des singularités ([Sc]) entraînent que $\rho \notin S S\left(\tilde{f}_{ \pm}\right)$. Supposons maintenant $\left|\tilde{\xi}-\tau / \cos \gamma_{ \pm}\right| \leq \varepsilon_{0}^{\prime}$. Alors, pour $\varepsilon_{0}^{\prime}>0$ assez petit, tout demirayon $\gamma$ issu de $\rho$ pouvant porter des singularités analytiques et dirigé vers $t<0$ est localement contenu dans $\{x>0\}$. Si $\varepsilon_{1}$ est assez petit, $\gamma$ possède un point dans $\{t<0\} \cap\{x>0\}$, où nous savons que $u_{r, \pm}$ est régulière. Le théorème de propagation des singularités analytiques pour le problème de Dirichlet de Sjöstrand [S1] entraîne donc, au vu de (3.106) et de (3.107), que $S S_{b}\left(u-u_{ \pm}\right) \cap \gamma=\varnothing$. Si $x>0$, on a donc $\rho \notin S S\left(\tilde{f}_{ \pm}\right)$. Si $x=0$, on en déduit que $u-u_{ \pm}$est $V_{ \pm}$-entrante si $\rho$ est hyperbolique. Si $\rho$ est glancing, i.e., $\rho=\left(0, t, \tau / \cos \gamma_{ \pm}, \tau\right)$, alors les demi-rayons issus de points proches de $\rho$ avec $x>0$ (dans $T^{*} \widetilde{\Delta}_{ \pm}$et dans $T^{*} D_{ \pm}$) restent dans $x>0$ avant d'entrer dans $t<0$, et le même argument de propagation des singularités entraîne que $u-u_{ \pm}$est $V_{ \pm}$-entrante au point $\rho$. $\Omega$,

En tenant compte de (3.102), la formule (2.7) devient, pour $(t, x, y)$ dans

$$
u=u_{e}-\square^{-1}\left(\tilde{f}_{+} \delta_{+}+\tilde{f}_{-} \delta_{-}\right),
$$

où $u_{e}=u_{i}-\square^{-1}\left(1_{x>0} \partial_{+} u_{+} \delta_{+}+1_{x>0} \partial_{-} u_{-} \delta_{-}\right)$est explicite. On est donc ramené à décrire microlocalement $\tilde{f}_{ \pm}$en $(t=x=0, \tau, \tilde{\xi})$, avec $\tilde{\xi} \neq-\tau / \cos \gamma_{ \pm}$. On utilisera le système suivant, issu de (2.34) et (3.102), avec $f_{ \pm}=\varphi_{1} \tilde{f}_{ \pm}$,

$$
\left\{\begin{array}{l}
f_{+}-1_{x>0} Q^{+} f_{+}-S^{+,-} f_{-}=h_{+}+r_{+}, \\
f_{-}-1_{x>0} Q^{-} f_{-}-S^{-{ }^{+}} f_{+}=h_{-}+r_{-},
\end{array}\right.
$$


où $h_{ \pm}$sont données par

(3.110)

$$
h_{ \pm}=1_{x>0}\left(2 \psi \varphi_{2} \partial_{ \pm} u_{i}-\varphi_{1} \partial_{ \pm} u_{ \pm}+Q^{ \pm}\left(1_{x>0} \varphi_{1} \partial_{ \pm} u_{ \pm}\right)+S^{ \pm, \mp}\left(1_{x>0} \varphi_{1} \partial_{\mp} u_{\mp}\right)\right)
$$

et où $r_{ \pm}$ont été définis en (2.35).

\section{MiCROLOCALISATION DU PROBLĖME ET DÉVELOPPEMENTS ASYMPTOTIQUES}

Rappelons que $f_{+}, f_{-}$ont été introduits en (3.109), le réel $t_{0}>0$ et les fonctions $\varphi_{1}, \varphi_{2}, \psi$ au $\S 2.3$, le réel $\kappa>0$ au $\S 2.1$ (2.15). Les fonctions $f_{ \pm}(x, t)$ sont $L^{2}$ et à support dans $0 \leq x \leq c t, t \leq 3 t_{0}$; on supposera de plus $\psi(x)=0$ pour $|x| \geq 4 c t_{0}, 0 \leq \psi \leq 1$.

Nous allons dans ce paragraphe effectuer une transformation FBI en variable $x$ sur le système (3.109) pour le ramener à un problème pseudo-différentiel à valeurs opérateurs. Les développements asymptotiques obtenus ici s'appuient sur les techniques usuelles de microlocalisation.

Le choix des différentes constantes intervenant dans la preuve étant assez laborieux, nous noterons toujours $D_{j}(j=1,2, \ldots)$ des constantes absolues, avec $D_{j} \geq 1$. On désignera par la même lettre $D$ des constantes vérifiant $D \geq 1$ et dont la valeur exacte est sans importance.

4.1. Transformation FBI en variable $x$. Soit $\psi_{2}(x) \in C_{0}^{\infty}$, égale à 1 sur le support de $\psi$, nulle pour $|x| \geq 5 c t_{0}$.

Pour $g \in L_{\text {loc }}^{2}(\mathbb{R})$, support $(g) \subset \mathbb{R}_{+}, z \in \mathbb{C}$, on pose

$$
T_{1} g(z, \lambda)=\int_{0}^{\infty} e^{\lambda x z-\lambda x^{2} / 2} \psi_{2}(x) g(x) d x .
$$

Si on définit la fonction poids $\tilde{\varphi}_{1}(z)$ par

$$
\left\{\begin{array}{l}
\tilde{\varphi}_{1}(z)=0, \quad \operatorname{Re} z \leq 0, \\
\tilde{\varphi}_{1}(z)=\frac{1}{2}(\operatorname{Re} z)^{2}, \quad \operatorname{Re} z \in\left[0,5 c t_{0}\right], \\
\tilde{\varphi}_{1}(z)=5 c t_{0}(\operatorname{Re} z)-\frac{1}{2}\left(5 c t_{0}\right)^{2}, \quad \operatorname{Re} z \geq 5 c t_{0},
\end{array}\right.
$$

on a les estimations

$$
\left\|\left(\frac{1}{\lambda} \partial_{z}\right)^{k} T_{1} g(x-i \alpha, \lambda) e^{-\lambda \tilde{\varphi}_{1}(x)}\right\|_{L^{2}(\alpha)} \leq D \lambda^{-1 / 2}\left(5 c t_{0}\right)^{k}\left\|\psi_{2} g\right\|_{L^{2}},
$$

et la formule d'inversion

$$
\psi_{2} g(x)=\frac{\lambda}{2 \pi} \int_{\mathbb{R}} e^{i \lambda x \alpha-\lambda x^{2} / 2} T_{1} g(x-i \alpha, \lambda) d \alpha,
$$

où l'intégrale est oscillante en $\alpha$. En appliquant la formule (4.4) à $g^{\prime}(x)=$ $e^{-i \lambda x \cdot \xi} g(x)$, avec $\xi \in \mathbb{C}$, on obtient

$$
e^{-i \lambda x \xi} \psi_{2} g(x)=\frac{\lambda}{2 \pi} \int_{\mathbb{R}} e^{i \lambda x \alpha-\lambda x^{2} / 2} T_{1} g(x-i \xi-i \alpha, \lambda) d \alpha .
$$


Soit $G=\left\{(t, x) \in \mathbb{R}^{2}, 0 \leq x \leq c t\right\}$. Pour $(w, z) \in \mathbb{C}^{2}$, on définit la fonction poids $\Phi$ par

$$
\sup _{G}-\frac{1}{2} \operatorname{Re}\left[(w-t)^{2}\right]+x \operatorname{Re} z-\frac{x^{2}}{2}=\frac{1}{2}(\operatorname{Im} w)^{2}+\Phi(\operatorname{Re} w, \operatorname{Re} z) .
$$

On obtient

$$
\left\{\begin{array}{l}
\Phi(v, u)=0 ; \quad v \geq 0, u \leq 0 \\
\Phi(v, u)=\frac{u^{2}}{2} ; \quad v \geq 0, \quad 0 \leq u \leq c v, \\
\Phi(v, u)=-\frac{1}{2} v^{2}+\frac{1}{2} \frac{(c u+v)^{2}}{1+c^{2}} ; \quad u \geq 0,-c u \leq v \leq \frac{u}{c} \\
\Phi(v, u)=-\frac{1}{2} v^{2} ; \quad v \leq 0, \quad u \leq-\frac{v}{c} .
\end{array}\right.
$$

Alors $\Phi(v, u)$ est croissante en $v$ et on posera, pour $\delta>0$,

$$
\Phi_{\delta}(u)=\Phi\left(\frac{\delta}{c}, u\right)
$$

d'où

$$
\left\{\begin{array}{l}
\Phi_{\delta}(u)=0 ; \quad u \leq 0 \\
\Phi_{\delta}(u)=\frac{u^{2}}{2} ; \quad u \in[0, \delta], \\
\Phi_{\delta}(u)=\frac{u^{2}}{2}-\frac{1}{2\left(1+c^{2}\right)}(u-\delta)^{2} ; \quad u \geq \delta
\end{array}\right.
$$

Rappelons que la transformation $T_{0}$ a été introduite au $\S 2.1$. Pour $f(t, x) \in L^{2}$ avec support $(f) \subset\left\{0 \leq x \leq c t ; 0 \leq t \leq 2 t_{0}\right\}$ on pose

$$
\begin{aligned}
F(w, z, \lambda) & =\left(T_{0} \otimes T_{1}\right)(f) \\
& =\int_{-\infty}^{+\infty} \int_{0}^{\infty} e^{\lambda\left(x z-x^{2} / 2-(w-t)^{2} / 2\right)} f(x, t) d x d t .
\end{aligned}
$$

On relie $\delta$ et $\kappa$ par

$$
\delta=3 \kappa c .
$$

En utilisant la définition de $\Phi, \Phi_{\delta}$, on obtient alors:

Lemme 4.1. Pour $w \in \mathbb{C}, \operatorname{Re} w \leq 3 \kappa$, et $x \in \mathbb{R}$ on $a$

$$
\left\{\begin{array}{l}
\left\|\left(\frac{1}{\lambda} \partial_{z}\right)^{k} F(w, x-i \alpha, \lambda)\right\|_{L^{2}\left(\mathbb{R}_{\alpha}\right)} \leq D\left(2 c t_{0}\right)^{k}\|f\|_{L^{2}} e^{\lambda(\operatorname{Im} w)^{2} / 2+\lambda \Phi_{\delta}(x)} \\
\left\|\left(\frac{1}{\lambda} \partial_{z}\right)^{k} F(w, x-i \alpha, \lambda)\right\|_{L^{\infty}\left(\mathbb{R}_{\alpha}\right)} \leq D\left(2 c t_{0}\right)^{k}\|f\|_{L^{2}} e^{\lambda(\operatorname{Im} w)^{2} / 2+\lambda \Phi_{\delta}(x)}
\end{array}\right.
$$


Posons

$$
W_{\kappa}=\{w \in \mathbb{C},|\operatorname{Re} w|<\kappa,|1+\operatorname{Im} w|<\kappa\}
$$

et fixons $\varepsilon_{0}>0$. D'après la Proposition 3.1, la caractérisation du deuxième micro-support à croissance par transformation FBI [L] et les estimées (4.12), on obtient:

Lemme 4.2. Il existe $\varepsilon_{1}>0, A, B, d>0$ tels que si $F_{ \pm}=\left(T_{0} \otimes T_{1}\right) f_{ \pm}$, on ait pour $\kappa \leq \varepsilon_{1}, w \in W_{3 \kappa}, z \in \mathbb{C}$,

$$
\left|F_{ \pm}(w, z, \lambda)\right| \leq A \lambda^{B} e^{\lambda(\operatorname{Im} w)^{2} / 2+\lambda \Phi_{\delta}^{ \pm}(z)}
$$

avec $\Phi_{\delta}^{ \pm}(z)=0$ pour $\operatorname{Re} z \in[0, d],\left|\operatorname{Im} z-1 / \cos \gamma_{ \pm}\right| \geq \varepsilon_{0},|z| \leq 10 /\left|\cos \gamma_{ \pm}\right|$, et $\Phi_{\delta}^{ \pm}(z)=\Phi_{\delta}(\operatorname{Re} z)$ ailleurs.

On prendra grade au fait que les constantes $\varepsilon_{1}, A, B, d$ dépendent de $t_{0}$, puisque $f_{ \pm}=\varphi_{1} \tilde{f}_{ \pm}$, et $\varphi_{1}$ dépend de $t_{0}$. On supposera dans la suite $d \leq c t_{0}$.

4.2. Espaces de fonctions holomorphes. Pour $A>0, \varepsilon>0$ petit, soit $\Gamma_{\varepsilon}=$ ]$\pi / 2+\varepsilon, 3 \pi / 2-\varepsilon\left[\right.$ et $\omega[\varepsilon, A]=\left\{z \in \mathbb{C} ; \operatorname{Arg}(z) \in \Gamma_{\varepsilon},|z| \geq A\right\}$. On dira qu'un ouvert $\omega$ connexe et simplement connexe de $\mathbb{C}$ est un $\varepsilon$-secteur s'il est de la forme $\omega=\omega[\varepsilon, A] \cup \omega^{\prime}$ avec $\omega^{\prime}$ ouvert borné. Si $\omega_{1}\left(\right.$ resp. $\left.\omega_{2}\right)$ est un $\varepsilon_{1}$ (resp. $\varepsilon_{2}$ )-secteur on écrira $\omega_{1} \Subset \omega_{2}$ si $\varepsilon_{1}>\varepsilon_{2}$ et $\bar{\omega}_{1} \subset \omega_{2}$.

Si $\omega$ est un $\varepsilon$-secteur, on note $H_{\omega}$ l'espace des fonctions $g(z, \lambda)$ définies pour $\lambda \geq \lambda_{0}$, holomorphes en $z \in \omega$, telles qu'on ait

$$
\exists C \quad \sup _{z \in \omega}|g(z, \lambda)|+\sup _{\theta \in \Gamma_{\varepsilon}}\left(\int_{A}^{\infty}\left|g\left(\rho e^{i \theta}, \lambda\right)\right|^{2} d \rho\right)^{1 / 2} \underset{\operatorname{def}}{=}\|g(\cdot, \lambda)\|_{\omega} \leq C \lambda^{C} .
$$

On dira que $g \in H_{\omega}$ est négligeable s'il existe $C>0$ telle que $\|g\|_{\omega} \leq C e^{-\lambda / C}$.

Pour $g(z, \lambda)$ holomorphe en $z, \rho>0$, on pose

$$
\begin{aligned}
J_{\rho}(g)(z, \lambda) & =\sqrt{\frac{\lambda}{2 \pi}} \int_{-\rho}^{+\rho} e^{-\lambda y^{2} / 2} g(z+y, \lambda) d y, \\
J_{\rho}^{-1}(g)(z, \lambda) & =\sqrt{\frac{\lambda}{2 \pi}} \int_{-\rho}^{+\rho} e^{-\lambda y^{2} / 2} g(z+i y, \lambda) d y
\end{aligned}
$$

(lorsque ces expressions ont un sens).

Alors pour $\omega^{\prime} \Subset \omega$, il existe $\rho_{0}>0$ tel que, pour $\left.\left.\rho \in\right] 0, \rho_{0}\right], J_{\rho}$ et $J_{\rho}^{-1}$ envoient $H_{\omega}$ dans $H_{\omega^{\prime}}$ et ces opérateurs sont indépendants de $\rho$ modulo un opérateur négligeable $R$, i.e., vérifiant

$$
\exists C, \quad \forall g \in H_{\omega}, \quad\|R(g)\|_{\omega^{\prime}} \leq C e^{-\lambda / C}\|g\|_{\omega} .
$$

Il existe aussi des opérateurs négligeables $R_{\rho}^{1,2}$ tels qu'on ait les égalités d'opérateurs de $H_{\omega}$ dans $H_{\omega^{\prime}}$

$$
J_{\rho} \circ J_{\rho}^{-1}=\mathrm{Id}+R_{\rho}^{1} ; \quad J_{\rho}^{-1} \circ J_{\rho}=\mathrm{Id}+R_{\rho}^{2} .
$$

On remarquera que $J_{\rho}$ est une quantification de la transformation canonique $(x, \xi) \mapsto(z, \zeta), z=x-i \xi, \zeta=\xi$, qui laisse stable la section nulle. 
Lemme 4.3. Soient $\rho>0$ et $\psi \in C_{0}^{\infty}(\mathbb{R}), \psi(x) \equiv 1$ près de $x=0$. Il existe $C>0$ tel que pour $z \in \mathbb{C}, \operatorname{Re} z<-1$ on ait

$$
\sqrt{\frac{\lambda}{2 \pi}} \int_{-\rho}^{\rho} e^{-\lambda y^{2} / 2} \frac{d y}{z+i y}=\lambda \int_{0}^{\infty} e^{\lambda x z-\lambda x^{2} / 2} \psi(x) d x+r(z, \lambda)
$$

avec

$$
|r(z, \lambda)| \leq \frac{C}{|z|} e^{-\lambda / C}
$$

Preuve. On a $\int_{0}^{\infty} e^{\lambda x z-\lambda x^{2} / 2}(\psi-1) d x=-\frac{1}{\lambda z} \int_{0}^{\infty} e^{\lambda x z-\lambda x^{2} / 2} g(x, \lambda) d x$ avec $g=$ $\psi^{\prime}(x)-\lambda x(\psi-1)$ nul près de $x=0$ et

$$
\left|\int_{\rho}^{\infty} e^{-\lambda y^{2} / 2} \frac{d y}{z+i y}\right| \leq \frac{1}{|z|} \int_{\rho}^{\infty} e^{-\lambda y^{2} / 2} d y \quad \text { si } \operatorname{Im} z \geq 0
$$

et

$$
\left|\int_{\rho}^{\infty} e^{-\lambda y^{2} / 2} \frac{d y}{z+i y}\right|=\left|\int_{\rho+\mathbb{R}_{+} e^{i \pi / 6}} e^{-\lambda y^{2} / 2} \frac{d y}{z+i y}\right| \leq \frac{1}{C|z|} e^{-\lambda / C} \text { si } \operatorname{Im} z \leq 0 .
$$

On peut donc pour vérifier (4.21) faire $\rho=\infty$ et $\psi \equiv 1$ dans (4.20), qui devient une égalité avec $r \equiv 0$.

Si $\omega$ est un $\varepsilon$-secteur, $X$ un ouvert de $\mathbb{C}^{2}, \varphi_{0}(w)=(\operatorname{Im} w)^{2} / 2$, on note $H\left(\varphi_{0}, X, \omega\right)$ l'espace des fonctions $g(t, \tau, z, \lambda)$ holomorphes en $(t, \tau) \in X$, à valeurs dans $H_{\omega}$ telles que

$$
\exists C, \quad \forall(t, \tau) \in X, \quad\|g(t, \tau, \cdot, \lambda)\|_{\omega} \leq C \lambda^{C} e^{\lambda \varphi_{0}(t-i \tau)} .
$$

On définit comme précédemment les éléments négligeables de $H\left(\varphi_{0}, X, \omega\right)$.

Soit alors $\left(\omega_{\sigma}\right)_{\sigma \in[0,1]}$ une famille de $\delta_{\sigma}$-secteurs, où $\delta_{\sigma}=(1-\sigma) \delta_{0}+\sigma \delta_{1}$, $0<\delta_{1}<\delta_{0}$, tels que, si $\sigma<\sigma^{\prime}$, $\omega_{\sigma} \Subset \omega_{\sigma^{\prime}}$, et, plus précisément, tels qu'il existe $d_{1}>0$ avec

$$
\sigma<\sigma^{\prime} \Rightarrow d\left(\omega_{\sigma}, \omega_{\sigma^{\prime}}^{c}\right)=d_{1}\left(\sigma^{\prime}-\sigma\right)
$$

On impose de plus qu'il existe des constantes $\varepsilon_{2}, c_{2}, d_{2}$, telles que pour tout $\sigma$

$$
\begin{gathered}
d\left(i, \omega_{\sigma}\right) \geq \varepsilon_{2}, \\
\forall y \in \mathbb{R}, \quad|y-1| \geq 2 \varepsilon_{2}, \quad|y| \leq 5, \quad d\left(i y, \omega_{\sigma}^{c}\right) \geq c_{2}, \\
\forall z \in \omega_{\sigma}, \quad \operatorname{Re} z \leq d_{2},
\end{gathered}
$$

de sorte que $\omega_{\sigma}$ ait l'allure représentée dans la Figure 3 (voir p. 376).

On demande de plus que, si $\varepsilon_{0}, d$ sont les constantes intervenant dans le Lemme 4.2 , on ait

$$
\varepsilon_{0} \cos \gamma_{ \pm}<\varepsilon_{2}, \quad d_{2} \cos \gamma_{ \pm}<d .
$$

On pose alors $H_{\sigma}=H_{\omega_{\sigma}}$. On introduit alors

$$
\omega_{\sigma}^{ \pm}=\frac{1}{\cos \gamma_{ \pm}} \omega_{\sigma}, \quad H_{\sigma}^{ \pm}=H_{\omega_{\sigma}^{ \pm}},
$$




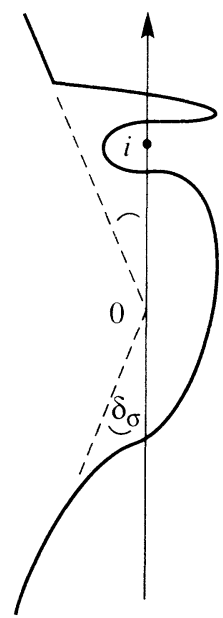

Figure 3

et on pose

$$
\|f\|_{\sigma}=\|f\|_{\omega_{\sigma}}, \quad\|f\|_{\sigma}^{ \pm}=\|f\|_{\omega_{\sigma}^{ \pm}} .
$$

En utilisant (4.23) et la croissance de $\left(\delta_{\sigma}\right)$, on vérifie alors aisément l'existence d'une constante $B>0$ telle que

$$
\text { (4.30) } \forall \sigma<\sigma^{\prime}, \quad \forall f \in H_{\sigma^{\prime}}, \quad \forall n \in \mathbb{N}, \quad\left\|\partial_{z}^{n} f\right\|_{\sigma} \leq B\left(\frac{B n}{\sigma^{\prime}-\sigma}\right)^{n}\|f\|_{\sigma^{\prime}}
$$

On a la même estimation pour les normes \|\|$_{\sigma}^{ \pm}$.

Dans ce qui suit, on supposera en outre que $\omega_{\sigma}$ a un bord $C^{1}$ par morceaux $\partial \omega_{\sigma}$.

4.3. Développement des termes pseudo-différentiels. Le but de ce paragraphe est de calculer le développement asymptotique en $\lambda$ de

$$
I(t, \tau, z, \lambda)=T_{1}\left(1_{x>0} Q_{\lambda \tau}^{+} T_{0} f_{+}\right)(t-i \tau, z, \lambda),
$$

où $Q_{\tau}^{+}$est l'opérateur défini en (2.36). On posera

$$
\sigma\left(x, x^{\prime}, \xi, \tau\right)=\left(\frac{\xi}{\xi_{+}}-\mu\right)\left(\frac{\mu-a^{\prime}(x)}{1+\mu^{2}}\right) .
$$

On fixe $A, \varepsilon$ avec $A \sin \varepsilon>4$ et on pose $U=\omega[\varepsilon, A]$. Pour $\kappa>0$, on posera

$$
X_{\kappa}=\left\{(t, \tau) \in \mathbb{C}^{2},|\tau-1|<3 \kappa, t-i \tau \in W_{3 \kappa}\right\} \text {. }
$$

Lemme 4.4. Il existe $D_{1}$ tel que, pour $\xi \in \mathscr{C}_{1}$, où $\mathscr{C}_{1}$ est le contour représenté dans la Figure 4 avec $a^{\prime} \ll t_{0} \ll 1 / D_{1}, a^{\prime \prime}=1+4 c t_{0}$, la fonction $\sigma\left(x, x^{\prime}, \tau, \xi\right)$ est holomorphe en $\left(x, x^{\prime}, \tau\right)$ pour $\max \left\{|\operatorname{Re} x|,\left|\operatorname{Re} x^{\prime}\right|\right\} \leq 5 c t_{0}$,

$$
\max \left\{|\operatorname{Im} x|,\left|\operatorname{Im} x^{\prime}\right|\right\} \leq 2 \frac{a^{\prime}}{D_{1}}, \quad|\tau-1|<4 \frac{a^{\prime}}{D_{1}},
$$

et vérifie $|\sigma| \leq D_{1} / a^{\prime}$. 


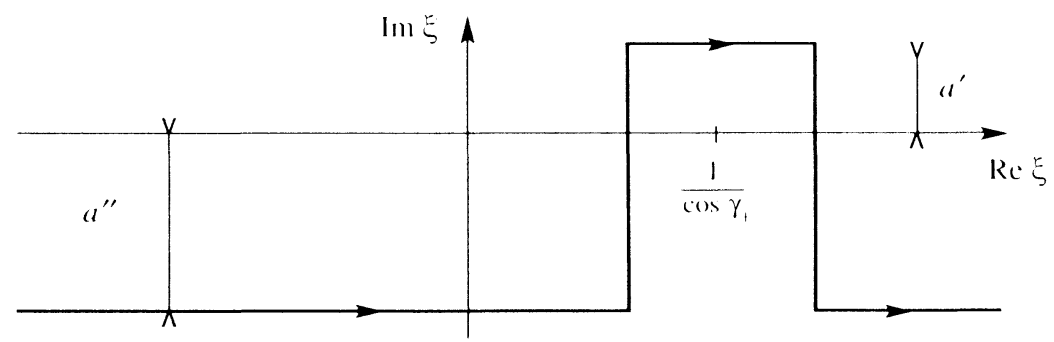

Figure 4

Preuve. Le lemme résulte de (4.32) et de $\xi_{+}=\left(\left(1+\mu^{2}\left(x, x^{\prime}\right)\right) \tau^{2}-\xi^{2}\right)^{1 / 2}$.

Proposition 4.1. Il existe $t_{0}^{1}$, et, pour $\left.t_{0} \in\right] 0, t_{0}^{1}\left[, a^{\prime}, \rho, c_{2}^{(0)}\right.$, tels que, avec $\kappa=a^{\prime} / D_{1}$, on ait $I \in H\left(\varphi_{0}, X_{\kappa}, U\right)$ et

$$
\begin{array}{r}
I-J_{\rho}^{-1}\left(\sum_{\substack{n \leq \lambda / C_{1} \\
m \leq \lambda / C_{2}}}\left(\frac{1}{\lambda} \partial_{z}\right)^{n} \frac{1}{2 \pi} \int_{\mathscr{C}_{1}} \frac{d \xi}{z+i \xi} \frac{\partial_{x}^{n} \partial_{x^{\prime}}^{m}}{n ! m !} \sigma(0,0, \tau, \xi)\right. \\
\left.\times\left(\frac{1}{\lambda} \partial_{z}\right)^{m} J_{\rho} F_{+}(-i \xi)\right)
\end{array}
$$

est négligeable dans $H\left(\varphi_{0}, X_{\kappa}, U\right)$, avec $C_{1} \geq e D_{1} / a^{\prime}, C_{2} \geq C_{2}^{0}$.

Les constantes seront choisies telles qu'on ait $t_{0}^{1} \ll 1, a^{\prime} \ll \inf \left(t_{0}, d, c_{2}\right)$ où $d$ est défini au Lemme 4.2. En particulier on aura $\kappa=a^{\prime} / D_{1} \ll \varepsilon_{1}$. Compte tenu des estimations (4.12), (4.14) et de $|z+i \xi| \geq 1$ pour $z \in U, \xi \in \mathscr{C}_{1}$, la série de (4.34) définit un élement de $H\left(\varphi_{0}, X_{\kappa}, U\right)$ ne dépendant de $\rho$ (petit) et de $C_{2}$ (grand) que modulo un terme négligeable, puisque l'opérateur de noyau $(z+i \xi)^{-n-1}$ est borné (uniformément en $n$ ) de $L^{2}\left(\mathscr{C}_{1}\right)$ dans $H_{U}$.

Pour la preuve de cette proposition, voir Appendice B.

D'après la Proposition 2.2, on a

$$
\begin{aligned}
T_{0} \otimes & T_{1}\left(1_{x \geq 0} Q^{+} f_{+}\right)(w, z, \lambda) \\
& =\frac{\lambda}{2 \pi} \int_{t_{-}}^{t_{+}} \int_{\tau_{-}}^{\tau_{+}} e^{i \lambda \tau(u)-t)-\lambda \tau^{2}} I(t, \tau, z, \lambda) d t d \tau+R
\end{aligned}
$$

où $R$ est négligeable dans $H_{\varphi_{0}}\left(W_{\kappa}, H_{U}\right)$, puisque $T_{1}$ envoie continûment $L^{2}$ dans $H_{U}$.

Par ailleurs, chaque intégrale dans la somme (4.34) peut être déformée sur le contour frontière de $i \omega_{\sigma}^{+}$:

$$
\mathscr{B}^{\sigma}=i \partial \omega_{\sigma}^{+} .
$$

Pour $|\tau-1|<3 k$ et $n \in \mathbb{N}$, considérons l'opérateur $q_{n}^{+}(\tau, \lambda): H_{0}^{+} \rightarrow H_{U}$ défini 
par

$$
\begin{aligned}
& q_{n}^{+}(\tau, \lambda) f(z) \\
& \quad=\sum_{j+m=n} \partial_{z}^{j}\left(\frac{1}{2 \pi} \int_{\mathscr{C}^{0}} \frac{d \xi}{z+i \xi} \frac{\partial_{x}^{j} \partial_{x^{\prime}}^{m}}{j ! m !} \sigma(0,0, \tau, \xi) \partial_{z}^{m} f(-i \xi) d \xi\right) .
\end{aligned}
$$

On notera que, compte tenu de (4.32), $q_{0}^{+}=0$. Alors, pour tous $\sigma, \sigma^{\prime} \in[0,1]$, $\sigma<\sigma^{\prime}$, on constate, en déformant $\mathscr{C}^{0}$ sur $\mathscr{C}^{\left(\sigma+\sigma^{\prime}\right) / 2}$, que $q_{n}^{+}$envoie $H_{\sigma^{\prime}}^{+}$dans $H_{\sigma}^{+}$, avec

$$
\left\|q_{n}^{+}(\tau, \lambda)\right\|_{\sigma, \sigma^{\prime}} \leq D\left(\frac{D n}{\sigma^{\prime}-\sigma}\right)^{n} .
$$

En effet, compte tenu de l'inégalité (4.30), il suffit d'étudier l'opérateur sur $H_{\sigma^{\prime}}^{+}$

$$
K_{n}: g \mapsto \frac{1}{2 \pi} \int_{\mathscr{C}\left(\sigma+\sigma^{\prime}\right) / 2} \frac{d \xi}{z+i \xi} \sigma_{n}(\tau, \xi) g(-i \xi)
$$

où $\sigma_{n}(\tau, \xi)=\left(\partial_{x}^{j} \partial_{x^{\prime}}^{m} / j ! m !\right) \sigma(0,0, \tau, \xi)$; les points de ramification de $\delta_{n}$ sont définis, d'après (4.32) et (2.26) par

$$
\xi^{2}=\frac{\tau^{2}}{\left(\cos \gamma_{+}\right)^{2}}
$$

et sur toute branche de détermination de $\sigma_{n}$ on a

$$
\begin{gathered}
\forall \varepsilon>0, \quad \exists D, \quad\left|\sigma_{n}(\tau, \xi)\right| \leq D^{n+1}, \quad \forall n, \\
|\tau-1| \leq 3 \varepsilon_{1}, \quad\left|\xi^{2}-\frac{\tau^{2}}{\left(\cos \gamma_{+}\right)^{2}}\right| \geq \varepsilon .
\end{gathered}
$$

Posons

$$
V_{\rho}=\left\{z \in \mathbb{C} ;\left|\operatorname{Im} z+\frac{1}{\cos \gamma_{+}}\right| \leq \frac{1}{\rho \cos \gamma_{+}},|\operatorname{Re} z| \leq \frac{c_{2}}{\rho \cos \gamma_{+}}\right\} .
$$

Pour $z \in V_{4}$, on a $\operatorname{dist}\left(z, \partial \omega_{.}^{+}\right) \geq c_{2} / 4 \cos \gamma_{+}$, donc

$$
\left|K_{n}(g)(z)\right| \leq D^{n+1}\|g\|_{\sigma^{\prime}}^{+} .
$$

Comme $|\tau-1| \leq 3 \kappa=3 a^{\prime} / D_{1} \ll \inf \left(t_{0}, d, c_{2}\right)$, les points de ramification de $\sigma_{n}(\tau, \xi)$ près de $\xi=1 / \cos \gamma_{+}$vérifient $-i \xi \in V_{8}$. Pour $z \notin V_{4}$ on déforme alors $\mathscr{C}^{\left(\sigma+\sigma^{\prime}\right) / 2}$ sur un contour entourant $V_{6}$, ayant l'allure suivante

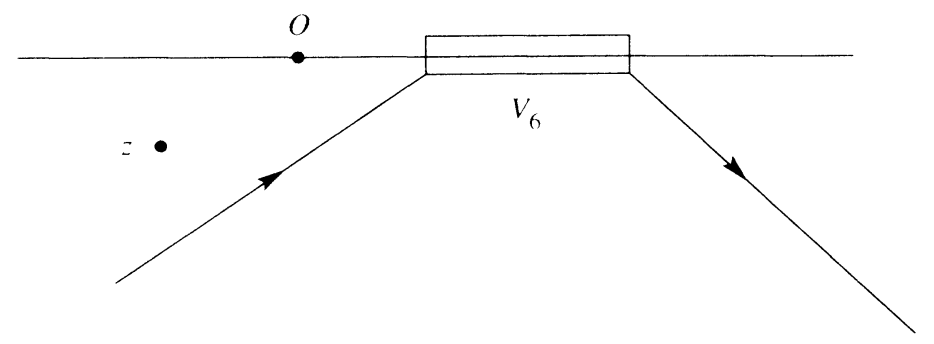




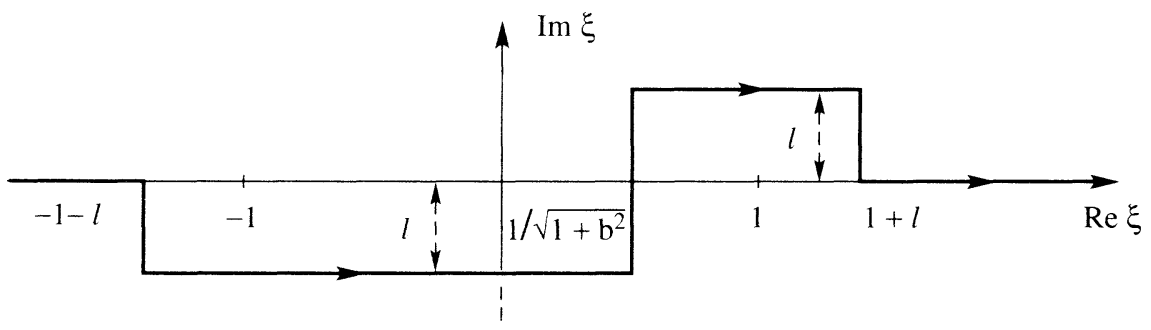

\section{FIGURE 5}

et pouvant faire apparaître un résidu au point $z$.

En utilisant l'inégalité de Hardy (Proposition 1.1), on obtient alors, compte tenu de (4.43)

$$
\exists D, \quad \forall \sigma \leq \sigma^{\prime}, \quad\left\|K_{n}\right\|_{\sigma, \sigma^{\prime}} \leq D^{n+1} .
$$

On a donc prouvé que le symbole

$$
q^{+}(\tau, \lambda)=\sum_{n=1}^{\infty} q_{n}^{+}(\tau, \lambda) \lambda^{-n}
$$

était un symbole à valeurs opérateurs dans la chaîne $H_{\sigma}^{+}$(au sens de l'Appendice A), et les formules (4.34) et (4.35) expriment que

$$
T_{0} \otimes T_{1}\left(1_{x \geq 0} Q^{+} f_{+}\right)(w, z, \lambda)=J_{\rho}^{-1} \mathrm{Op}\left(q_{+}\right) J_{\rho} F_{+}
$$

modulo un élément négligeable de $H_{\varphi_{0}}\left(W_{\kappa}, H_{U}\right)$.

4.4. Développement des termes singuliers. Dans ce paragraphe, on étudie la transformée FBI de $S^{+,-} f_{-}$pour $w \in W_{\kappa}, z \in U$. D'après la Proposition 2.2 , on sait que

$$
\begin{aligned}
& \left(T_{1} \otimes T_{0}\right)\left(S^{+,-} f_{-}\right)(w, z, \lambda) \\
& \quad-\frac{\lambda}{2 \pi} \int_{t_{-}}^{t_{+}} d t \int_{\tau_{-}}^{\tau_{+}} d \tau e^{i \lambda \tau(w-t)-\lambda \tau^{2}} T_{1} S_{\lambda \tau}^{+,-} T_{0} f_{-}(t-i \tau, \lambda)
\end{aligned}
$$

est négligeable dans $H_{\varphi_{0}}\left(W_{\kappa}, H_{U}\right)$.

Pour $l>0, \kappa \ll l \ll 1$, on introduit le contour $\mathscr{C}(l, b)$ représenté dans la Figure 5. On a alors le résultat suivant (voir Appendice B).

Proposition 4.2. Il existe $t_{0}^{1}>0$, et, pour tout $\left.\left.t_{0} \in\right] 0, t_{0}^{1}\right], l, \kappa_{0}, C_{1}^{0}, C_{2}^{0}$, tels que, pour tout $\left.\kappa \in] 0, \kappa_{0}\right]$, on ait

$$
\widetilde{I}=T_{1}\left(S_{\lambda \tau}^{+} T_{0} f_{-}\right)(t-i \tau, z, \lambda) \in H\left(\varphi_{0}, X_{\kappa}, U\right),
$$

et

$$
\begin{aligned}
& \widetilde{I}+\left.\sum_{\substack{n \leq \lambda / C_{1} \\
m \leq \lambda / C_{2}}}\left(\frac{1}{\lambda} \partial_{z}\right)^{n} \frac{1}{2 \pi} \int_{\mathscr{E}(l, b)} \frac{d \xi}{z+i\left(\xi+a \xi_{0}\right)} \frac{\partial_{x}^{n}}{n !}\left(e^{\lambda \phi_{+}}\left(1-a^{\prime}(x) \frac{\xi}{\xi_{0}}\right)\right)\right|_{x=0} \\
& \times\left.\frac{\partial_{x^{\prime}}^{m}}{m !}\left(e^{\lambda \phi}-\left(\frac{1}{\lambda} \partial_{z}\right)^{m} F_{-}\right)\right|_{x^{\prime}=0, z=-i\left(\xi+h \xi_{0}\right)}
\end{aligned}
$$


est négligeable dans $H\left(\varphi_{0}, X_{\kappa}, U\right)$, où l'on a noté

$$
\begin{gathered}
F_{-}=F_{-}\left(t-i \tau, x^{\prime}+z, \lambda\right), \\
\phi_{+}=i(a(x)-a x) \xi_{0}-\frac{x^{2}}{2}, \\
\phi_{-}=-i\left(b\left(x^{\prime}\right)-b x^{\prime}\right) \xi_{0}-\frac{x^{\prime 2}}{2},
\end{gathered}
$$

et où $C_{1} \geq C_{1}^{0}, C_{2} \geq C_{2}^{0}$.

Pour $n \in \mathbb{N}$ et $|\tau-1|<3 \kappa$, considérons l'opérateur $s_{n}^{+,-}(\tau, \lambda): H_{0}^{-} \rightarrow H_{U}$ défini par

$$
\begin{aligned}
s_{n}^{+,-}(\tau, \lambda) f \\
=-\left.\sum_{j+m=n} \partial_{z}^{j} \frac{1}{2 \pi} \int_{\mathscr{C}(l, b)} \frac{d \xi}{z+i\left(\xi+a \xi_{0}\right)} \frac{\partial_{x}^{j}}{j !}\left(e^{\lambda \phi_{+}}\left(1-a^{\prime}(x) \frac{\xi}{\xi_{0}}\right)\right)\right|_{x=0} \\
\times \frac{\left.\partial_{x^{\prime}}^{m}\left(e^{\lambda \phi_{-}} \partial_{z}^{m} f\left(x^{\prime}+z\right)\right)\right|_{x^{\prime}=0, z=-i\left(\xi+b \xi_{0}\right)}}{m !}
\end{aligned}
$$

Pour étudier cet opérateur, déformons le contour $\mathscr{C}(l, b)$ sur le contour

$$
\mathscr{C}_{b}, \quad \xi=\frac{\zeta-b \zeta_{0}}{\sqrt{1+b^{2}}}=\zeta \cos \gamma_{-}-\zeta_{0} \sin \gamma_{-},
$$

où $\zeta$ décrit le contour $\Gamma$ suivant, supposé contenu dans $\bar{\omega}_{0}$, avec $\delta>\delta_{0}$, $\left.\gamma=\gamma_{+}-\gamma_{-} \in\right] 0, \pi[$

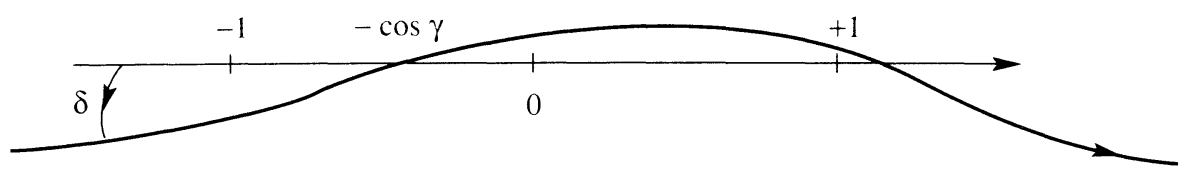

Ce contour $\Gamma$ est précisé dans l'Appendice $C$.

Le contour $\mathscr{C}_{b}$ a l'allure représentée dans la Figure 6 et on a, puisque $\xi_{0}=$ $\sin \gamma_{-} \zeta+\cos \gamma_{-} \zeta_{0}$,

$$
\xi+b \xi_{0}=\frac{1}{\cos \gamma_{-}}\left(\xi \cos \gamma_{-}+\xi_{0} \sin \gamma_{-}\right)=\frac{\zeta}{\cos \gamma_{-}} \in \bar{\omega}_{0}^{-}
$$

tandis que,

$$
\begin{aligned}
\xi+a \xi_{0} & =\frac{1}{\cos \gamma_{+}}\left(\xi \cos \gamma_{+}+\xi_{0} \sin \gamma_{+}\right) \\
& =\frac{1}{\cos \gamma_{+}}\left(\zeta \cos \gamma+\zeta_{0} \sin \gamma\right), \quad \zeta \in \Gamma,
\end{aligned}
$$




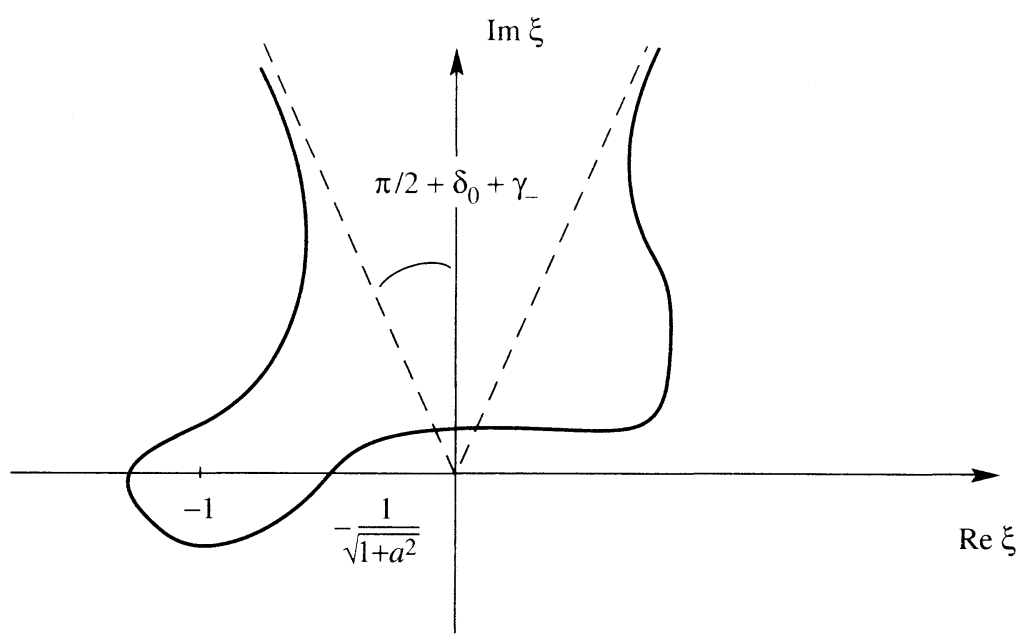

Figure 6

décrit un contour d'allure suivante

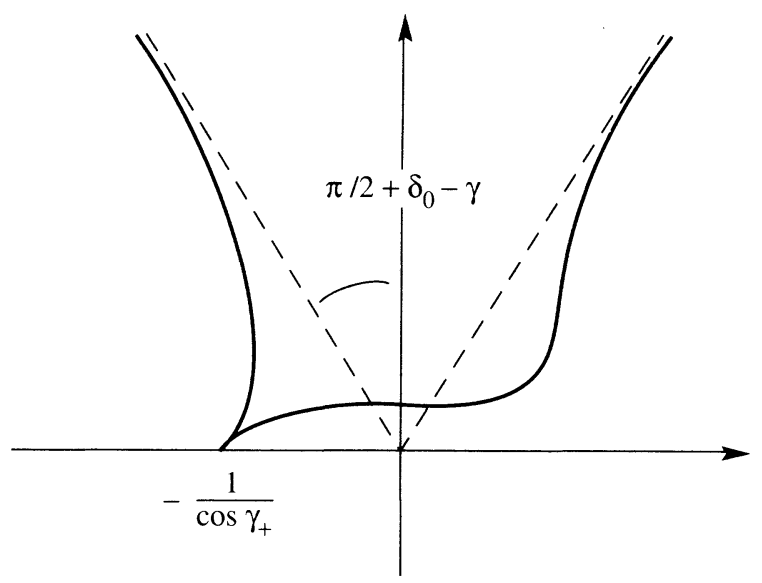

On montre dans l'Appendice C que l'on peut choisir la famille $\left(\omega_{\sigma}\right)$ de sorte que le contour $\Gamma$ soit contenu dans $i \bar{\omega}_{0}$ et que le contour (4.57) défini par (4.56) reste à l'extérieur de $i \bar{\omega}_{1}^{+}$. On en déduit que

$$
\begin{gathered}
s_{n}^{+,-}(\tau, \lambda): H_{0}^{-} \rightarrow H_{1}^{+}, \\
\left\|s_{n}^{+,-}(\tau, \lambda)\right\|_{1,0} \leq D^{n+1} n ! .
\end{gathered}
$$

Alors la série formelle

$$
s^{+,-}(\tau, \lambda)=\sum_{n=0}^{\infty} s_{n}^{+,-}(\tau, \lambda) \lambda^{-n}
$$

est un symbole analytique à valeurs dans l'espace des opérateurs bornés de $H_{0}^{-}$ 
dans $H_{1}^{+}$, et la Proposition 4.2 jointe à la formule (4.48) expriment que

$$
\left(T_{1} \otimes T_{0}\right)\left(S^{+,-} f_{-}\right)=\mathrm{Op}\left(s^{+,-}\right) F_{-}+R,
$$

où $R$ est négligeable dans $H_{\varphi_{0}}\left(W_{\kappa}, H_{U}\right)$.

4.5. Développement du second membre. On donne maintenant le développement de la transformée FBI du second membre de (3.109). Notons que, d'après le Lemme 2.2.2, l'image par $T_{0} \otimes T_{1}$ d'une fonction supportée dans $t \geq t_{0}$ est négligeable dans $H_{\varphi_{0}}\left(W_{\kappa}, H_{U}\right)$. Modifiant $h_{+}$par une telle fonction, on est ramené à etudier $T_{0} \otimes T_{1}\left(h_{+}\right)$, avec, selon les notations du $\S 3.3$,

$$
h_{+}=1_{x \geq 0}\left[s_{1}+Q_{+}\left(1_{x \geq 0} s_{2}\right)+S^{+,-}\left(s_{3}\right)\right] \text {, }
$$

avec

$$
\begin{gathered}
s_{j}(x, t)=\frac{1}{2 \pi} \int_{-\infty}^{+\infty} e^{i \tau\left(t-\theta_{a}(x)\right)} p_{j}(x, \tau) d \tau, \quad j=1,2 \\
s_{3}(x, t)=\frac{1}{2 \pi} \int_{-\infty}^{+\infty} e^{i \tau\left(t-\theta_{b}(x)\right)} p_{3}(x, \tau) d \tau \\
\theta_{a}(x)=\theta_{i}(x, a(x)), \quad \theta_{b}(x)=\theta_{i}(x, b(x)) \\
p_{1}(x, \tau)=\partial_{+} \sigma_{i}(x, \tau)+\partial_{+} \sigma_{+}(x, \tau), \\
p_{2}(x, \tau)=-2 i \tau \partial_{+} \theta_{i}(x) \sigma_{i}(x, a(x), \tau)+\partial_{+} \sigma_{i}(x, \tau)-\partial_{+} \sigma_{+}(x, \tau) \\
p_{3}(x, \tau)=-2 i \tau \partial_{-} \theta_{i}(x) \sigma_{i}(x, b(x), \tau)+\partial_{-} \sigma_{i}(x, \tau)-\partial_{-} \sigma_{-}(x, \tau) .
\end{gathered}
$$

Etudions d'abord la contribution de $s_{1}$. On a

$$
\begin{aligned}
T_{0} & \otimes \\
& T_{1}\left(1_{x \geq 0} s_{1}\right) \\
& =\sqrt{\frac{\lambda}{2 \pi}} \int_{-\infty}^{+\infty} e^{i \lambda w \tau-\lambda \tau^{2} / 2} \int_{0}^{\infty} \psi_{2}(x) e^{\lambda x z-\lambda x^{2} / 2-i \lambda \tau \theta_{a}(x)} p_{1}(x, \lambda \tau) d x d \tau .
\end{aligned}
$$

Puisque $\operatorname{Re} z<0$ dans $U$, on peut remplacer le domaine d'intégration en $\tau$ par $[1-2 \kappa, 1+2 \kappa]$, puis développer l'intégrale en $x$ pour obtenir

$$
\begin{aligned}
T_{0} \otimes & T_{1}\left(1_{x \geq 0} s_{1}\right) \\
\sim & \sqrt{\frac{\lambda}{2 \pi}} \int_{1-2 \kappa}^{1+2 \kappa} e^{i \lambda w \tau-\lambda \tau^{2} / 2} \sum_{n \leq \lambda / C} \lambda^{-n-1} \\
& \times\left.\left(\frac{-1}{z-i \tau}\right)^{n+1} \partial_{x}^{n}\left(e^{-\lambda x^{2} / 2-i \lambda \tau \tilde{\theta}_{a}(x)} p_{1}(x, \lambda \tau)\right)\right|_{x=0}
\end{aligned}
$$

modulo un élément négligeable de $H_{\varphi_{0}}\left(W_{\kappa}, H_{U}\right)$, avec $\tilde{\theta}_{a}(x)=\theta_{a}(x)-\theta_{a}^{\prime}(0) x=$ $\theta_{a}(x)-x$. On posera de même $\tilde{\theta}_{b}(x)=\theta_{b}(x)-x$.

Les deux autres termes font l'objet de la proposition suivante, démontrée dans l'Appendice B. 
Proposition 4.3. On pose

$$
I_{2}(t, \tau, z, \lambda)=T_{1}\left(1_{x \geq 0} Q_{\lambda \tau}^{+}\left(1_{x \geq 0} s_{2}\right)\right)(t-i \tau, z, \lambda),
$$

et

$$
I_{3}(t, \tau, z, \lambda)=T_{1}\left(S_{\lambda \tau}^{+,-}\left(s_{3}\right)\right)(t-i \tau, z, \lambda) .
$$

Alors il existe $t_{0}^{1}>0$, et, pour tout $\left.\left.t_{0} \in\right] 0, t_{0}^{1}\right], \kappa, a^{\prime}, l, C_{1}^{0}, C_{2}^{0}, \rho$, tels que, pour $C_{1} \geq C_{1}^{0}, C_{2} \geq C_{2}^{0}$, on ait, modulo un élément négligeable de $H_{\varphi_{0}}\left(W_{\kappa}, H_{U}\right)$,

$$
\begin{aligned}
I_{2} \sim \sqrt{\frac{\lambda}{2 \pi}} \int_{1-4 \kappa}^{1+4 \kappa} e^{i \lambda(t-i \tau) \tau^{\prime}-\lambda \tau^{\prime 2} / 2} \times \frac{1}{2 \pi} \int_{\mathscr{C}_{1}} j_{2}\left(t, \tau, z, \tau^{\prime}, \xi, \lambda\right) d \xi d \tau^{\prime} \\
j_{2}=J_{\rho}^{-1} \sum_{\substack{n \leq \lambda / C_{1} \\
m \leq \lambda / C_{2}}}\left(\frac{1}{\lambda} \partial_{z}\right)^{n}\left(\frac{1}{z+i \xi}\right) \frac{\lambda^{-m-1}}{\left(i\left(\xi+\tau^{\prime}\right)\right)^{m+1}} \\
\times\left.\frac{\partial_{x}^{n}}{n !} \partial_{x^{\prime}}^{m}\left(\sigma\left(x, x^{\prime}, \xi, \tau\right) p_{2}\left(x^{\prime}, \lambda \tau^{\prime}\right) e^{-i \lambda \tau \tilde{\theta}_{a}\left(x^{\prime}\right)}\right)\right|_{x=x^{\prime}=0}
\end{aligned}
$$

$$
I_{3} \sim \sqrt{\frac{\lambda}{2 \pi}} \int_{1-4 \kappa}^{1+4 \kappa} e^{i \lambda(t-i \tau) \tau^{\prime}-\lambda \tau^{\prime 2} / 2} \frac{1}{2 \pi} \int_{\mathscr{C}(l, b)} j_{3}\left(t, \tau, z, \tau^{\prime}, \xi, \lambda\right) d \xi d \tau^{\prime}
$$

$$
\begin{aligned}
j_{3}=-\sum_{\substack{n \leq \lambda / C_{1} \\
m \leq \lambda / C_{2}}}\left(\frac{1}{\lambda} \partial_{z}\right)^{n} & \left(\frac{1}{z+i\left(\xi+a \xi_{0}\right)}\right) \frac{\lambda^{-m-1}}{\left(i\left(\xi+b \xi_{0}+\tau^{\prime}\right)\right)^{m+1}} \\
& \times\left.\frac{\partial_{x}^{n}}{n !}\left(e^{\lambda \phi_{+}}\left(1-a^{\prime}(x) \frac{\xi}{\xi_{0}}\right)\right)\right|_{x=0} \\
& \times\left.\partial_{x^{\prime}}^{m}\left(e^{\lambda \phi_{-}-i \lambda \tau^{\prime} \dot{\theta}_{h}\left(x^{\prime}\right)} p_{3}\left(x^{\prime}, \lambda \tau^{\prime}\right)\right)\right|_{x^{\prime}=0} .
\end{aligned}
$$

Par la Proposition 2.2, on a par ailleurs, modulo des éléments négligeables dans $H_{\varphi_{0}}\left(W_{\kappa}, H_{U}\right)$,

$$
\begin{gathered}
\left(T_{0} \otimes T_{1}\right)\left(1_{x \geq 0} Q^{+}\left(1_{x \geq 0} S_{2}\right)\right) \sim \frac{\lambda}{2 \pi} \int_{t_{-}}^{t_{+}} d t \int_{\tau_{-}}^{\tau_{+}} d \tau e^{i \lambda(u-t) \tau-\lambda \tau^{2}} I_{2}, \\
\left(T_{0} \otimes T_{1}\right)\left(S^{+,-}\left(s_{3}\right)\right) \sim \frac{\lambda}{2 \pi} \int_{t_{-}}^{t_{+}} d t \int_{\tau_{-}}^{\tau_{+}} d \tau e^{i \lambda(u-t) \tau-\lambda \tau^{2}} I_{3}
\end{gathered}
$$

Nous allons maintenant comparer le développement asymptotique de $T_{0} \otimes$ $T_{1}\left(h_{+}\right)$ainsi obtenu dans $H_{\varphi_{0}}\left(W_{\kappa}, H_{U}\right)$ à un élément de $H_{\varphi_{0}}\left(W_{\kappa}, H_{1}^{+}\right)$. Dans les développements qui suivent, $C$ désignera une constante assez grande, dont on se réserve la possibilité d'augmenter la valeur, et $r$ un élément négligeable dans $H_{\varphi_{0}}\left(W_{\kappa}, H_{U}\right)$. 
Commençons par (4.70), et appliquons à l'intégrale en $\tau$ la formule de la phase stationnaire suivante

$$
\sqrt{\frac{\lambda}{2 \pi}} \int_{1-2 \kappa}^{1+2 \kappa} e^{i \lambda \tau w-\lambda \tau^{2} / 2} q(\tau) d \tau \sim e^{-\lambda w^{2} / 2} \sum_{k \leq \lambda / C}(2 \lambda)^{-k} \frac{1}{k !} \partial_{\tau}^{2 k} q(i w),
$$

modulo un élément négligeable de $H_{\varphi_{0}}\left(W_{\kappa}\right)$. En regroupant les puissances de $(z+w)^{-1}$ ainsi obtenues, on a

$$
T_{0} \otimes T_{1}\left(1_{x \geq 0} s_{1}\right)=e^{-\lambda w^{2} / 2} \sum_{n \leq \lambda / C} \frac{1}{(z+w)^{n+1}} s_{1, n}(w, \lambda)+r,
$$

$$
n \leq \frac{\lambda}{C} \Rightarrow\left|s_{1, n}(w, \lambda)\right| \leq D \lambda^{D} \theta(C)^{n}, \quad \theta(C) \underset{C \rightarrow+\infty}{\longrightarrow} O^{+} .
$$

Passons au développement donné par (4.71), (4.72), (4.75). On écrit

$$
\int_{\mathscr{C}_{1}} j_{2} d \xi=\int_{\mathscr{C}_{2}} j_{2} d \xi+2 i \pi j_{2}^{\prime}
$$

où $\mathscr{C}_{2}$ est le contour suivant,

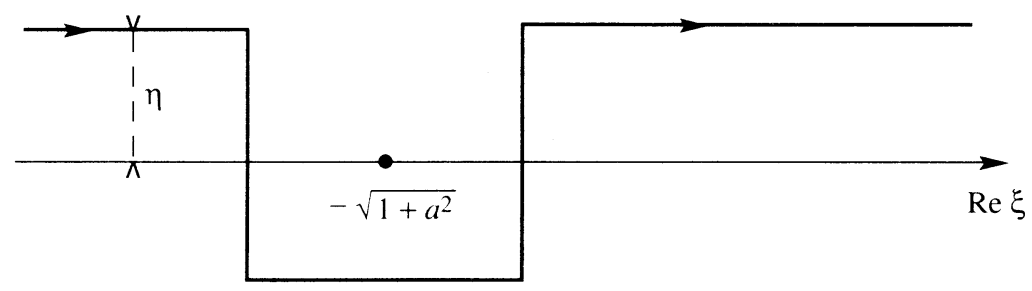

avec $\eta \ll 1$, de sorte que $\mathscr{C}_{2}$ soit à l'extérieur de $\omega_{1}^{+}$, et où $j_{2}^{\prime}$ est le résidu de $j_{2}$ en $\xi=-\tau^{\prime}$, soit

$$
\begin{aligned}
j_{2}^{\prime}= & J_{\rho}^{-1} \sum_{\substack{n \leq \lambda / C_{1} \\
m \leq \lambda / C_{2}}}\left(\frac{1}{\lambda} \partial_{z}\right)^{n}\left(\frac{1}{i \lambda} \partial_{\xi}\right)^{m} \\
& \times\left.\left(\frac{(i \lambda)^{-1}}{z+i \xi} \frac{\partial_{x}^{n}}{n !} \frac{\partial_{x^{\prime}}^{m}}{m !}\left(\sigma\left(x, x^{\prime}, \xi, \tau\right) p_{2}\left(x^{\prime}, \lambda \tau^{\prime}\right) e^{-i \lambda \tau^{\prime} \tilde{\theta}_{a}}\right)\right)\right|_{x=x^{\prime}=0, \xi=-\tau^{\prime}} .
\end{aligned}
$$

Notons

$$
\phi\left(w, t, \tau, \tau^{\prime}\right)=\tau(w-t)+i \tau^{2}+\tau^{\prime}(t-i \tau)+i \frac{\tau^{\prime 2}}{2},
$$

et désignons par $\Sigma$ le contour en $t, \tau, \tau^{\prime}$

$$
\Sigma: t \in\left[t_{-}, t_{+}\right], \quad \tau \in\left[\tau_{-}, \tau_{+}\right], \quad \tau^{\prime} \in[1-4 \kappa, 1+4 \kappa] .
$$

La valeur critique de $\phi$ étant $i w^{2} / 2$, on pose

$$
\varphi_{1}(w)=-\operatorname{Im}\left(i \frac{w^{2}}{2}\right)=\frac{1}{2}\left[(\operatorname{Im} w)^{2}-(\operatorname{Re} w)^{2}\right] .
$$


Alors

$$
\left(\frac{\lambda}{2 \pi}\right)^{3 / 2} \iiint_{\Sigma} e^{i \lambda \phi}\left(\int_{\mathscr{C}_{2}} j_{2} \frac{d \xi}{2 \pi}\right) d t d \tau d \tau^{\prime}=l_{2}^{+}(w, z, \lambda),
$$

où $l_{2}^{+} \in H_{\varphi_{1}}\left(W_{\kappa}, H_{1}^{+}\right)$, comme on le voit en déformant $\Sigma$ sur un bon contour $\widetilde{\Sigma}$ passant par le col $\tau=\tau^{\prime}=i w, t=0$. vient

Par ailleurs, en utilisant la formule de la phase stationnaire en $\left(t, \tau, \tau^{\prime}\right)$, il

$$
\begin{aligned}
& \left(\frac{\lambda}{2 \pi}\right)^{3 / 2} \iiint_{\Sigma} e^{i \lambda \phi} i j_{2}^{\prime} d t d \tau d \tau^{\prime} \\
& =e^{-\lambda w^{2} / 2} \sum_{n \leq \lambda / C} J_{\rho}^{-1}\left(\frac{1}{(z+w)^{n+1}}\right) s_{2, n}(w, \lambda)+r
\end{aligned}
$$

pour $w \in W_{\kappa}$. En remarquant que, modulo un terme négligeable dans $H_{0}\left(W_{\kappa}, H_{U}\right)$, on a

$$
J_{\rho}^{-1}\left(\frac{1}{(z+w)^{n+1}}\right) \sim \sum_{k \leq \lambda / C} \frac{(-1)^{k}}{k ! n !}(n+2 k) !(2 \lambda)^{-k} \frac{1}{(z+w)^{n+1+2 k}},
$$

on peut encore écrire (4.87) sous la forme

$$
\begin{aligned}
& \left(\frac{\lambda}{2 \pi}\right)^{3 / 2} \iiint_{\Sigma} e^{i \lambda \phi} i j_{2}^{\prime} d t d \tau d \tau^{\prime} \\
& =e^{-\lambda w^{2} / 2} \sum_{n \leq \lambda / C} \frac{1}{(z+w)^{n+1}} \tilde{s}_{2, n}(w, \lambda)+r,
\end{aligned}
$$

où $\tilde{s}_{2, n}$ vérifie $(4.88)$.

Passons enfin au développement donné par (4.73), (4.74), (4.76). On déforme dans (4.73) l'intégrale en $\xi$ sur le contour $\mathscr{C}(l, a)$ défini par

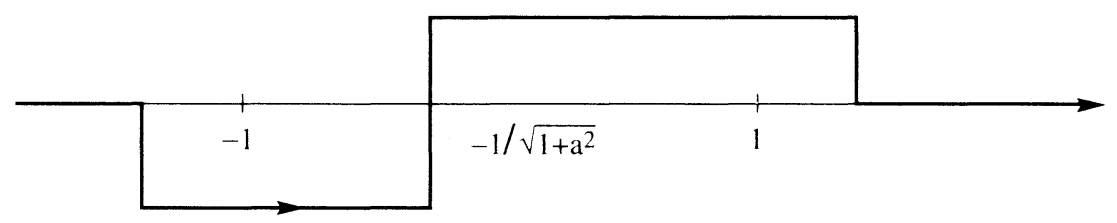

En remarquant que, dans le feuillet de $\xi_{0}$ où l'on fait la déformation, la solution de l'équation $\xi+b \xi_{0}=-\tau$ ' se trouve dans un voisinage d'ordre $\kappa$ de $\xi=-1$, on conclut que la déformation ne fait pas apparaître de terme résiduel si $l \ll 1$. On en déduit que

$$
\begin{aligned}
& \left(T_{0} \otimes T_{1}\right)\left(S^{+,-}\left(s_{3}\right)\right)(w, z, \lambda)=l_{3}^{+}(w, z, \lambda)+r \\
& l_{3}^{+}=\left(\frac{\lambda}{2 \pi}\right)^{3 / 2} \iiint_{\Sigma} e^{i \lambda \phi} \frac{1}{2 \pi} \int_{\mathscr{C}(l, a)} j_{3} d \xi d t d \tau d \tau^{\prime},
\end{aligned}
$$


et une déformation de $\Sigma$ sur $\widetilde{\Sigma}$ assure que $l_{3}^{+} \in H_{\varphi_{1}}\left(W_{\kappa}, H_{1}^{+}\right)$.

En conclusion, on peut écrire

$$
\begin{aligned}
\left(T_{0} \otimes T_{1}\right)\left(h_{+}\right)(w, z, \lambda)= & l_{2}^{+}(w, z, \lambda)+l_{3}^{+}(w, z, \lambda) \\
& +\sum_{n \leq \lambda / C} \frac{r_{n}(w, \lambda)}{(z+w)^{n+1}}+r
\end{aligned}
$$

où $r$ est négligeable dans $H_{\varphi_{0}}\left(W_{\kappa}, H_{U}\right)$, et où

$$
n \leq \frac{\lambda}{C} \Rightarrow\left|r_{n}(w, \lambda)\right| \leq D \lambda^{D} e^{\lambda \varphi_{1}(w)} \theta(C)^{n}, \quad \theta(C) \underset{C \rightarrow+\infty}{\longrightarrow} O^{+}
$$

4.6. Système microlocalisé. Appliquons maintenant l'opérateur $T_{0} \otimes T_{1}$ à la première équation du système (3.109). Compte tenu des identités (4.47), (4.61), et (4.94), et en utilisant le Lemme 2.2 pour traiter le cas de $r_{+}$, on obtient

$$
R(w, z, \lambda)+\sum_{n \leq \lambda / C} \frac{r_{n}(w, \lambda)}{(z+w)^{n+1}}=\widetilde{R}(w, z, \lambda),
$$

où $\widetilde{R}$ est négligeable dans $H_{\varphi_{0}}\left(W_{\kappa}, H_{U}\right)$, les $r_{n}$ satisfont à $(4.95)$, et où

$$
R=F_{+}-J_{\rho}^{-1} \mathrm{Op}\left(q^{+}\right) J_{\rho} F_{+}-\mathrm{Op}\left(s^{+,-}\right) F_{-}-l_{2}^{+}-l_{3}^{+} \text {. }
$$

Or les estimations sur $F_{ \pm}$, l'étude des opérateurs $q^{+}$et $s^{+,-}$faite aux $\S \S 4.3$ et 4.4 , et les estimations sur $l_{2}^{+}, l_{3}^{+}$obtenues en $\S 4.5$ permettent d'affirmer que

$$
R \in H_{\varphi_{0}}\left(W_{\kappa}, H_{1}^{+}\right) \text {. }
$$

Fixons par ailleurs $\alpha>0$ petit tel que le disque fermé $D$ de centre $i$ et de rayon $\alpha$ soit contenu dans $\omega_{1}^{+}$, et posons $\tilde{\omega}=\omega_{1}^{+} \backslash D$. Alors pour $\kappa$ assez petit, $-W_{\kappa} \subset D$, et, compte tenu des estimations (4.95) sur les $r_{n}$, on a, si $C$ est assez grand pour que $\theta(C)<\alpha$,

$$
\widetilde{R} \in H_{\varphi_{0}}\left(W_{\kappa}, H_{\tilde{\omega}}\right) .
$$

En appliquant le principe du maximum [L2] à la fonction holomorphe

$$
z \in \tilde{\omega} \mapsto \widetilde{R}(w, z, \lambda)
$$

on conclut que $\widetilde{R}$ est négligeable dans $H_{\varphi_{0}}\left(W_{\kappa}, H_{\tilde{\omega}^{\prime}}\right)$ pout tout $\tilde{\omega}^{\prime} \Subset \tilde{\omega}$; en particulier, si $C$ est un cercle de centre $i$ et de rayon $\beta>\alpha$ contenu dans $\tilde{\omega}$, on a, pour tout $n \in \mathbb{N}$

$$
\left|\frac{1}{2 i \pi} \int_{C}(z+w)^{n} \widetilde{R}(w, z, \lambda) d z\right| \leq D e^{\lambda \varphi_{0}\left(w^{\prime}\right)-\lambda / D},
$$

soit, en revenant à (4.96), et en utilisant (4.98),

$$
\left|r_{n}(w, \lambda)\right| \leq D e^{\lambda \varphi_{0}(u)-\lambda / D}
$$

Mais alors (4.96) entraîne que $R$ est négligeable dans $H_{\varphi_{0}}\left(W_{\kappa}, H_{U}\right)$. En choisissant l'angle $\delta_{1}$, associé à $\omega_{1}^{+}$supérieur à l'angle $\varepsilon$ associè à $U$, on constate 
que $\omega_{1}^{+} \backslash U$ est borné, et, en appliquant à nouveau le principe du maximum [L2] à $z \in \omega_{1}^{+} \mapsto R(w, z, \lambda)$, on conclut que $R$ est négligeable dans $H_{\varphi_{0}}\left(W_{\kappa}, H_{\sigma}^{+}\right)$ pour tout $\sigma<1$.

On peut évidemment reprendre les $\S \S 4.3,4.4,4.5$ et l'argument précédent pour la seconde équation de (3.109). On obtient finalement le système suivant:

$$
\left\{\begin{array}{l}
F_{+}-J_{\rho}^{-1} \mathrm{Op}\left(q^{+}\right) J_{\rho} F_{+}-\mathrm{Op}\left(s^{+,-}\right) F_{-}=l_{2}^{+}+l_{3}^{+}+R^{+}, \\
F_{-}-J_{\rho}^{-1} \mathrm{Op}\left(q^{-}\right) J_{\rho} F_{-}-\mathrm{Op}\left(s^{-,}, F_{+}=l_{2}^{-}+l_{3}^{-}+R^{-},\right.
\end{array}\right.
$$

où $R^{ \pm}$sont négligeables dans $H_{\varphi_{0}}\left(W_{\kappa}, H_{\sigma}^{ \pm}\right), \forall \sigma<1, F_{ \pm} \in H_{\varphi_{0}}\left(W_{\kappa}, H_{1}^{ \pm}\right)$, $q^{+}, s^{+,-}$et $l_{2}^{+}, l_{3}^{+}$ont été définis en $((4.37),(4.46)),((4.52),(4.60))$, et $((4.72)$, $(4.86)),((4.74),(4.93))$ respectivement. On a de même:

$$
q^{-}(\tau, \lambda)=\sum_{n=1}^{\infty} q_{n}^{-}(\tau, \lambda) \lambda^{-n}
$$

où $q_{n}^{-}$est donné par (4.37), en remplaçant $\sigma$ par

$$
\sigma^{\prime}=\left(\frac{\xi}{\xi_{-}}+\nu\right)\left(\frac{b^{\prime}(x)-\nu}{1+\nu^{2}}\right)
$$

et où $\mathscr{C}^{0}$ est remplacé par $i \partial \omega_{0}^{-}$de sorte que $q_{n}^{-}$est un symbole à valeurs opérateurs dans la chaîne $H_{\sigma}^{-}$,

$$
s^{-,+}(\tau, \lambda)=\sum_{n=0}^{\infty} s_{n}^{-,+}(\tau, \lambda) \lambda^{-n}
$$

$$
\begin{aligned}
& s_{n}^{-,+}(\tau, \lambda) f(z) \\
& =-\left.\sum_{j+m=n} \partial_{z}^{j} \frac{1}{2 \pi} \int_{\mathscr{C}_{-a}} \frac{d \xi}{z+i\left(\xi-b \xi_{0}\right)} \frac{\partial_{x}^{j}}{j !}\left(e^{\lambda \phi_{-}}\left(1+b^{\prime}(x) \frac{\xi}{\xi_{0}}\right)\right)\right|_{x=0} \\
& \times\left.\frac{\partial_{x^{\prime}}^{m}}{m !}\left(e^{\lambda \phi_{+}} \partial_{z}^{m} f\left(x^{\prime}+z\right)\right)\right|_{x^{\prime}=0, z=-i\left(\xi-a \xi_{0}\right)},
\end{aligned}
$$

où les phases $\phi_{ \pm}$ont été définies en (4.51), et où $\mathscr{C}_{-a}$ désigne le contour suivant:

$$
\mathscr{C}_{-a}: \quad \xi=\frac{\zeta+a \zeta_{0}}{\sqrt{1+a^{2}}}=\zeta \cos \gamma_{+}+\zeta_{0} \sin \gamma_{+},
$$

où $\zeta$ décrit le contour $\Gamma$ défini en (4.54).

Ainsi on vérifie que $s^{-,+}$est un symbole à valeurs opérateurs de $H_{0}^{+}$dans $H_{1}^{-}$. Enfin, $l_{2}^{-}, l_{3}^{-}$sont donnés par

$$
l_{2}^{-}(w, z, \lambda)=\left(\frac{\lambda}{2 \pi}\right)^{3 / 2} \iiint_{\Sigma} e^{i \lambda \phi}\left(\int_{\mathscr{F}_{2}^{\prime}} j_{4} \frac{d \xi}{2 \pi}\right) d t d \tau d \tau^{\prime}
$$




$$
\begin{aligned}
j_{4}=J_{\rho}^{-1} \sum_{\substack{n \leq \lambda / C_{1} \\
m \leq \lambda / C_{2}}} & \left(\frac{1}{\lambda} \partial_{z}\right)^{n}\left(\frac{1}{z+i \xi}\right) \frac{\lambda^{-m-1}}{\left(i\left(\xi+\tau^{\prime}\right)\right)^{m+1}} \frac{\partial_{x}^{n}}{n !} \\
& \times\left.\partial_{x^{\prime}}^{m}\left(\sigma^{\prime}\left(x, x^{\prime}, \xi, \tau\right) p_{3}\left(x^{\prime}, \lambda \tau^{\prime}\right) e^{-i \lambda \tilde{\theta}_{b}\left(x^{\prime}\right)}\right)\right|_{x=x^{\prime}=0},
\end{aligned}
$$

où $\mathscr{C}_{2}^{\prime}$ est le contour (4.81), a étant remplacé par $b, \phi$ est définie en (4.83),

$$
\begin{gathered}
l_{3}^{-}(w, z, \lambda)=\left(\frac{\lambda}{2 \pi}\right)^{3 / 2} \iiint_{\Sigma} e^{i \lambda \phi}\left(\int_{\mathscr{E}(l,-b)} j_{5} \frac{d \xi}{2 \pi}\right) d t d \tau d \tau^{\prime}, \\
j_{5}=-\sum_{\substack{n \leq \lambda / C_{1} \\
m \leq \lambda / C_{2}}}\left(\frac{1}{\lambda} \partial_{z}\right)^{n}\left(\frac{1}{z+i\left(\xi-b \xi_{0}\right)}\right) \frac{\lambda^{-m-1}}{\left(i\left(\xi-a \xi_{0}+\tau^{\prime}\right)\right)^{m+1}} \frac{\partial_{x}^{n}}{n !} \\
\times\left.\left(e^{\lambda \phi_{-}}\left(1+b^{\prime}(x) \frac{\xi}{\xi_{0}}\right)\right)\right|_{x=0} \times\left.\partial_{x^{\prime}}^{m}\left(e^{\lambda \phi_{+}-i \lambda \tau^{\prime} \hat{\theta}_{a}\left(x^{\prime}\right)} p_{2}\left(x^{\prime}, \lambda \tau^{\prime}\right)\right)\right|_{x^{\prime}=0},
\end{gathered}
$$

où $\mathscr{C}(l,-b)$ est le contour (4.91), $a$ étant remplacé par $b$.

On a $l_{2}^{-}, l_{3}^{-} \in H_{\varphi_{1}}\left(W_{\kappa}, H_{1}^{-}\right)$. Posons alors

$$
G_{ \pm}=J_{\rho} F_{ \pm}, \quad L_{ \pm}=J_{\rho}\left(l_{2}^{ \pm}+l_{3}^{ \pm}+R^{ \pm}\right), \quad \tilde{s}^{ \pm, \mp}=J_{\rho} s^{ \pm, \mp} J_{\rho}^{-1} .
$$

Pour $\rho$ assez petit, on a

$$
\begin{aligned}
& G_{ \pm} \in H_{\varphi_{0}}\left(W_{\kappa}, H_{2 / 3}^{ \pm}\right), \\
& L_{ \pm} \in H_{\varphi_{1}}\left(W_{\kappa}, H_{2 / 3}^{ \pm}\right), \tilde{s}^{ \pm, \mp}: H_{1 / 3}^{\mp} \rightarrow H_{2 / 3}^{ \pm}
\end{aligned}
$$

de plus, (4.103) devient

$$
\left\{\begin{array}{l}
G_{+}-\mathrm{Op}\left(q^{+}\right) G_{+}-\mathrm{Op}\left(\tilde{s}^{+,-}\right) G_{-}=L_{+} \\
G_{-}-\mathrm{Op}\left(q^{-}\right) G_{-}-\operatorname{Op}\left(\tilde{s}^{+,-}\right) G_{+}=L_{-}
\end{array}\right.
$$

qui est un système pseudo-différentiel à valeurs opérateurs dans la chaîne $H_{\sigma}^{+} \oplus$ $H_{\sigma}^{-}, \sigma \in[1 / 3,2 / 3]$. Compte tenu des formules précédentes, le symbole principal de ce système est $I-\widetilde{A}(\tau, \lambda)$ où

$$
\tilde{A}(\tau, \lambda)=J_{\rho}\left(\begin{array}{cc}
0 & s_{0}^{+,-}(\tau) \\
s_{0}^{-,+}(\tau) & 0
\end{array}\right) J_{\rho}^{-1},
$$

et en posant

$$
M_{ \pm} f(z)=f\left(\cos \gamma_{ \pm} z\right)
$$

de sorte que $M_{ \pm}: H_{\sigma} \rightarrow H_{\sigma}^{ \pm}$, on a

$$
s_{0}^{ \pm, \mp}(\tau)=M_{ \pm} S(\tau) M_{\mp}^{-1},
$$

$$
S(\tau) g(z)=-\frac{1}{2 \pi} \int_{\Gamma} \frac{\cos \gamma-\zeta \sin \gamma / \zeta_{0}}{z+i\left(\zeta \cos \gamma+\zeta_{0} \sin \gamma\right)} g(-i \zeta) d \zeta
$$


Enfin, en vue d'identifier, à la $\S 6$, le terme principal de $F_{+}$, il est utile de calculer celui de $L_{ \pm}$, à l'aide des formules $((4.86),(4.72)),((4.93),(4.74))$, $((4.109),(4.110)),((4.111),(4.112)),(4.67),(4.68)$, soit, après avoir éliminé les variables $t, \tau, \tau^{\prime}$ par la formule de la phase stationnaire,

$$
\begin{gathered}
L_{ \pm}^{0}=J_{\rho}\left(m_{ \pm}\right) \sigma_{i}(0,0, i \lambda w) e^{-\lambda w^{2} / 2} \\
m_{+}(w, z)=\frac{1}{2 \pi} \int_{\mathscr{C}(l, a)} \frac{1-a \xi / \xi_{0}}{z+i\left(\xi+a \xi_{0}\right)} \frac{2 b}{\xi+b \xi_{0}+i w} d \xi \\
m_{-}(w, z)=-\frac{1}{2 \pi} \int_{\mathscr{C}(l,-b)} \frac{1+b \xi / \xi_{0}}{z+i\left(\xi-b \xi_{0}\right)} \frac{2 a}{\xi-a \xi_{0}+i w} d \xi
\end{gathered}
$$

où $\xi_{0}$ est relatif à $\tau=i w$, i.e., $\xi_{0}=\left(-w^{2}-\xi^{2}\right)^{1 / 2}$.

\section{INVERSION DU SYMBOLE PRINCIPAL}

Le but de cette section est de prouver que le système pseudo-différentiel (4.115) est elliptique, ce qui, compte tenu de la Proposition A.3 de l'Appendice A, équivaut à prouver que l'opérateur $I-\widetilde{A}(\tau, \lambda)$ est inversible de $H_{\sigma^{\prime}}^{+} \oplus H_{\sigma^{\prime}}^{-}$ dans $H_{\sigma}^{+} \oplus H_{\sigma}^{-} \quad\left(\sigma^{\prime}>\sigma\right)$ pour tout $\tau$ dans un voisinage de 1 , et pour $\lambda$ assez grand. En fait, compte tenu des propriétés régularisantes de $\widetilde{A}(\tau, \lambda)$ on peut travailler à l'intérieur d'un même espace $H_{\sigma}^{+} \oplus H_{\sigma}^{-}$, par exemple $H_{0}^{+} \oplus H_{0}^{-}$. Enfin, les formules (4.116), (4.117), et (4.118) montrent que l'on est ramené à étudié l'inversibilité sur $H_{0} \oplus H_{0}$ de l'opérateur $I-A(\tau)$, où

$$
A(\tau)=\left(\begin{array}{cc}
0 & S(\tau) \\
S(\tau) & 0
\end{array}\right)
$$

et $S(\tau)$ est donné par (4.119). Notons que, comme les notations le suggèrent, $S(\tau)$ ne dépend pas de $\lambda$. On peut donc supposer dans ce paragraphe que les éléments de l'espace $H_{0}$ sont des fonctions holomorphes indépendantes de $\lambda$. Enfin l'argument usuel de perturbation permet de supposer $\tau=1$, ce que nous ferons dans tout ce paragraphe. On a donc $\xi_{0}=\left(1-\xi^{2}\right)^{1 / 2}$.

Désignons par $L$ l'espace des fonctions $\varphi$ holomorphes dans $\operatorname{Re} z<0$, vérifiant

$$
\sup _{s<0} \int_{-\infty}^{+\infty}|\varphi(s+i \zeta)|^{2} d \zeta<+\infty .
$$

Le théorème de Paley-Wiener affirme que $L$ coïncide avec les fonctions de la forme

$$
\varphi(z)=\int_{0}^{\infty} e^{z x} \Phi(x) d x
$$

où $\Phi \in L^{2}\left(\mathbb{R}_{+}\right)$.

Si $\alpha \in \mathbb{R}$ et $\varphi$ est une fonction holomorphe sur un domaine $D, R_{\alpha} \varphi$ désigne la fonction holomorphe sur le domaine $e^{-i \alpha} D$ définie par

$$
R_{\alpha} \varphi(z)=\varphi\left(e^{i \alpha} z\right)
$$




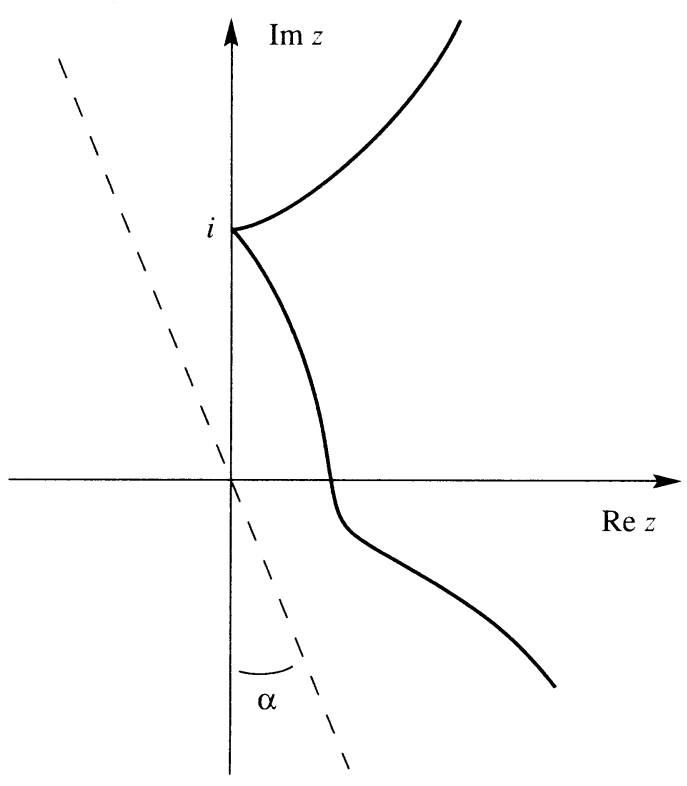

Figure 7

Lemme 5.1. Il existe $\alpha \in] 0, \pi / 2\left[\right.$ tel que l'opérateur $R_{\alpha} S(1)$ soit borné de $H_{0}$ dans $L$.

Démonstration. Reprenons l'expression (4.119) avec $\tau=1$. Lorsque $\zeta$ décrit $\Gamma$, le point $z=-i\left(\zeta \cos \gamma+\zeta_{0} \sin \gamma\right)$ décrit un contour du type représenté dans la Figure 7.

Il existe $\alpha \in] 0, \pi / 2[$ tel que ce contour ne rencontre pas le demi-plan $e^{i \alpha}\{\operatorname{Re} z \leq 0\}$. On en déduit le Lemme 5.1.

On va utiliser le lemme précédent pour déformer le problème initial sur un problème elliptique. Soit $\alpha$ comme ci-dessus, et soit $g \in H_{0}$ telle que $R_{\alpha} g \in$ $L$. En déformant le contour $\Gamma$ sur le contour $e^{i \alpha} \mathbb{R}$, on obtient

$$
R_{\alpha} S(1) g=S_{\alpha} R_{\alpha} g,
$$

où

$$
S_{\alpha} \varphi(z)=-\frac{1}{2 \pi} \int_{-\infty}^{+\infty} \frac{\left(\cos \gamma-\zeta \sin \gamma / \zeta_{\alpha}\right)}{z+i\left(\zeta \cos \gamma+\zeta_{\alpha} \sin \gamma\right)} \varphi(-i \zeta) d \zeta
$$

avec $\zeta_{\alpha}=\left(e^{-2 i \alpha}-\zeta^{2}\right)^{1 / 2}, \operatorname{Im} \zeta_{\alpha}>0$.

L'intérêt du système $I-A_{\alpha}$, avec $A_{\alpha}=\left(\begin{array}{cc}0 & S_{\alpha} \\ S_{\alpha} & 0\end{array}\right)$ est qu'il est aisément inversible sur $L$, car il s'exprime comme un problème elliptique sur l'ouvert de $\mathbb{R}^{2}, \mathscr{O}=\mathbb{R}^{2} \backslash\{y=a x, x \geq 0\} \backslash\{y=b x, x \geq 0\}$. En effet, si $\varphi_{ \pm} \in L$, définissons $\Phi_{ \pm} \in L^{2}(\mathbb{R}), \operatorname{supp} \Phi_{ \pm} \subset \mathbb{R}_{+}$, par

$$
\varphi_{ \pm}(-i \xi)=\widehat{\Phi}_{ \pm}\left(\frac{\xi}{\cos \gamma_{ \pm}}\right)
$$


en vertu de la formule (5.3). Alors on vérifie aisément la formule

$$
\frac{1}{2} S_{\alpha} \varphi_{\mp}(-i \xi)=\mathscr{F}\left[1_{x>0} \partial_{ \pm}\left(\Delta+e^{-2 i \alpha}\right)^{-1}\left(\Phi_{\mp} \delta_{\mp}\right)\right]\left(\frac{\xi}{\cos \gamma_{ \pm}}\right),
$$

où $\partial_{ \pm} \psi(x)= \pm\left.\left(\partial_{y}-\operatorname{tg} \gamma_{ \pm} \partial_{x}\right) \psi\right|_{y-\operatorname{tg} \gamma_{ \pm} x=0^{ \pm}}$.

On introduit de façon analogue $\partial_{ \pm}^{\prime}$ par

$$
\partial_{ \pm}^{\prime} \psi(x)= \pm\left.\left(\partial_{y}-\operatorname{tg} \gamma_{ \pm} \partial_{x}\right) \psi\right|_{y-\operatorname{tg} \gamma_{ \pm} x=0^{\mp}} .
$$

Considérons le système

$$
\left\{\begin{array}{l}
\left(\Delta+e^{-2 i \alpha}\right) U=0 \quad \text { dans } \mathscr{O}, \\
\left.U\right|_{y-\operatorname{tg} \gamma_{ \pm} x=0^{+}}=\left.U\right|_{y-\operatorname{tg} \gamma_{ \pm} x=0^{-}}, \\
\partial_{ \pm}^{\prime} U=-\frac{\Phi_{ \pm}}{2} .
\end{array}\right.
$$

Le Théorème D.1 de l'Appendice $\mathrm{D}$, assure que (5.8) est bien posé dans le sous-espace $E$ de $H^{1}\left(\mathbb{R}^{2}\right)$ défini en coordonnées polaires par

$$
\begin{gathered}
U \in C_{\theta}\left(\mathbb{R} / 2 \pi \mathbb{Z}, H_{r}^{1}\left(\mathbb{R}_{+}\right)\right), \\
\frac{1}{r} \partial_{\theta} U \in C_{\theta}\left(\left[\gamma_{-}, \gamma_{+}\right], L^{2}\left(\mathbb{R}_{+}\right)\right) \cap C_{\theta}\left(\left[\gamma_{+}, 2 \pi+\gamma_{-}\right], L^{2}\left(\mathbb{R}_{+}\right)\right) .
\end{gathered}
$$

On pose alors

$$
\Psi_{ \pm}=1_{x>0}\left(\partial_{ \pm}-\partial_{ \pm}^{\prime}\right) U \in L^{2}\left(\mathbb{R}_{+}\right),
$$

de sorte que, dans $\mathbb{R}^{2}$ tout entier,

$$
\left(\Delta+e^{-2 i \alpha}\right) U=\Psi_{+} \delta_{+}+\Psi_{-} \delta_{-} .
$$

En posant

$$
\psi_{ \pm}(-i \xi)=\widehat{\Psi}_{ \pm}\left(\frac{\xi}{\cos \gamma_{ \pm}}\right)
$$

et en appliquant la formule (5.7) à $\psi_{ \pm}$, on en déduit

$$
\psi_{ \pm}-\frac{\varphi_{ \pm}}{2}=\frac{1}{2} \psi_{ \pm}+\frac{1}{2} S_{c x} \psi_{\mp}
$$

soit encore

$$
\left(I-A_{\alpha}\right)\left(\begin{array}{c}
\psi_{+} \\
\psi_{-}
\end{array}\right)=\left(\begin{array}{c}
\varphi_{+} \\
\varphi_{-}
\end{array}\right)
$$

Inversement, si $\psi_{ \pm}$est solution de (5.11), la fonction

$$
U=\left(\Delta+e^{-2 i \alpha}\right)^{-1}\left(\Psi_{+} \delta_{+}+\Psi_{-} \delta_{-}\right)
$$

est solution du système (5.8), en remontant les calculs. On a donc prouvé le: 
Lemme 5.2. Le système (5.11) est bien posé dans $L \times L$.

Passons à l'inversion de $I-A(1): H_{0} \oplus H_{0} \rightarrow H_{0} \oplus H_{0}$. Montrons d'abord l'injectivité. Si $g=\left(g_{+}, g_{-}\right)$vérifie

$$
(I-A(1)) g=0 \text {, }
$$

alors $g=A(1) g$ donc $R_{\alpha} g \in L$ par le Lemme 5.1, et on peut écrire, en vertu de (5.4),

$$
R_{\alpha} A(1) g=A_{\alpha} R_{\alpha} g .
$$

En appliquant $R_{\alpha}$ à (5.12), on en déduit

$$
\left(I-A_{\alpha}\right) R_{\alpha} g=0 \text {, }
$$

et le Lemme 5.2 entraîne que $R_{\alpha} g=0$, donc $g=0$.

Soit maintenant $h=\left(h_{+}, h_{-}\right) \in H_{0} \oplus H_{0}$. Posons

$$
g_{1}=R_{-\alpha}\left(I-A_{\alpha}\right)^{-1} R_{\alpha} A(1) h .
$$

A priori $g_{1} \in R_{-\alpha}(L) \oplus R_{-\alpha}(L)$ seulement, et n'est donc pas dans $H_{0} \oplus H_{0}$. Cependant, on a le:

Lemme 5.3. Pour $k \in R_{-\alpha}(L)$, on définit

$$
S(1) k(z)=R_{-\alpha} S_{\alpha} R_{\alpha} k \in R_{-\alpha}(L) .
$$

Alors, si $n$ est un entier tel que $n \geq \pi / 2 \gamma$, on a

$$
S(1)^{n}: R_{-\alpha}(L) \rightarrow H_{0} .
$$

Supposons ce lemme prouvé, et terminons la construction de $(I-A(1))^{-1} h$. On pose, pour $n$ comme dans le Lemme 5.3,

$$
g \cdot=h+A(1) h+\cdots+A(1)^{n} h+A(1)^{n} g_{1} .
$$

Alors $g \in H_{0} \times H_{0}$ d'après le Lemme 5.3, et on a, grâce à une déformation de $\Gamma \operatorname{sur} \mathbb{R} e^{i \alpha}$,

$$
g-A(1) g=h-A(1)^{n+1} h+A(1)^{n}\left(g_{1}-A(1) g_{1}\right) .
$$

Par construction,

$$
g_{1}-A(1) g_{1}=R_{-\alpha}\left(I-A_{\alpha}\right)\left(I-A_{\alpha}\right)^{-1} R_{\alpha} A(1) h=A(1) h,
$$

donc $g-A(1) g=h$.

En résumé, on aura donc prouvé la:

Proposition 5.1. L'opérateur $I-A(1): H_{0} \times H_{0} \rightarrow H_{0} \times H_{0}$ est inversible, et

$$
(I-A(1))^{-1}=\sum_{\kappa=0}^{n} A(1)^{\kappa}+A(1)^{n} R_{-\alpha}\left(I-A_{\alpha}\right)^{-1} R_{\alpha} A(1),
$$

où $n$ est un entier $\geq \pi / 2 \gamma$, et où $\left(I-A_{\alpha}\right)^{-1}$ est décrit par les relations (5.11), (5.6), (5.10), (5.9), (5.8).

Il reste à prouver le Lemme 5.3. Pour $k \in R_{-\alpha}(L)$, on a

$$
S(1) k(z)=-\frac{1}{2 \pi} \int_{\mathbb{R} e^{i u}} \frac{\left(\cos \gamma-\zeta \sin \gamma / \zeta_{0}\right)}{z+i\left(\xi \cos \gamma+\zeta_{0} \sin \gamma\right)} k(-i \zeta) d \zeta .
$$




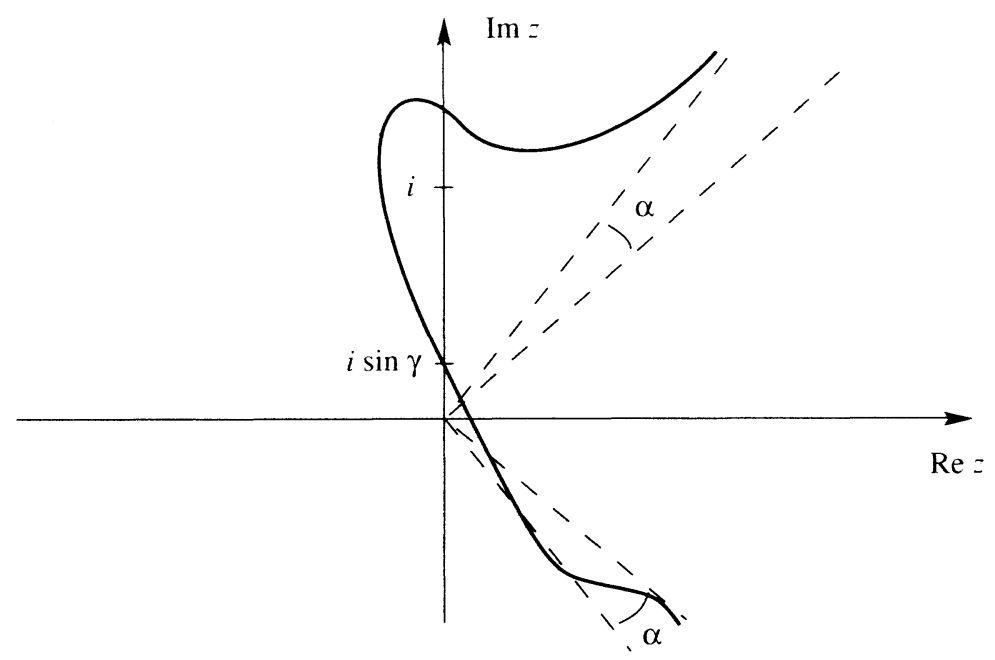

Figure 8

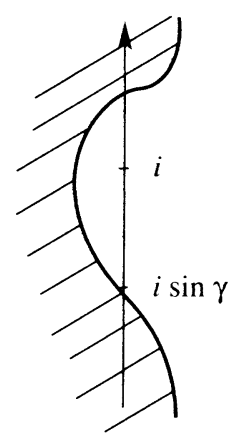

Figure 9

Supposons d'abord $\gamma<\pi / 2$. Lorsque $\zeta$ décrit $\mathbb{R} e^{i \alpha}$, on constate que $z=$ $-i\left(\zeta \cos \gamma+\zeta_{0} \sin \gamma\right)$ décrit un contour du type representé dans la Figure 8.

On en déduit que $S(1) k$ est holomorphe, $L^{2}$ à l'infini, sur un domaine du type représenté dans la Figure 9.

L'épaisseur de ce domaine dans $\operatorname{Re} z>0$ peut être supposée grande par rapport à celle de $\omega_{0}$.

Si $\sin \gamma \geq \cos \gamma$, on peut alors calculer $S(1)^{2} k$ au moyen du contour $\Gamma$ (voir (4.54)), et conclure que $S(1)^{2} k \in H_{0}$.

Sinon, on calcule $S(1)^{2} k$ avec le contour en $\zeta$ representé dans la Figure 10, p. 394.

En général supposons que $l$ soit holomorphe, $L^{2}$ à l'infini, dans un domaine du type representé dans la Figure 11, p. 394, avec $\theta \in[0, \pi / 2-\gamma$ [. Alors, en intégrant sur le contour en $\zeta$ représenté sur la Figure 12, p. 394, on constate que $S(1) l$ est holomorphe dans un domaine du même type, $\theta$ étant remplacé par $\theta+\gamma$. On en déduit que, si $n \gamma<\pi / 2, S(1)^{n} R_{-\alpha}$ envoie $L$ dans les fonctions holomorphes dans un domaine de ce type, avec $\theta=n \gamma$. Si $\sin n \gamma \geq \cos \gamma$, 


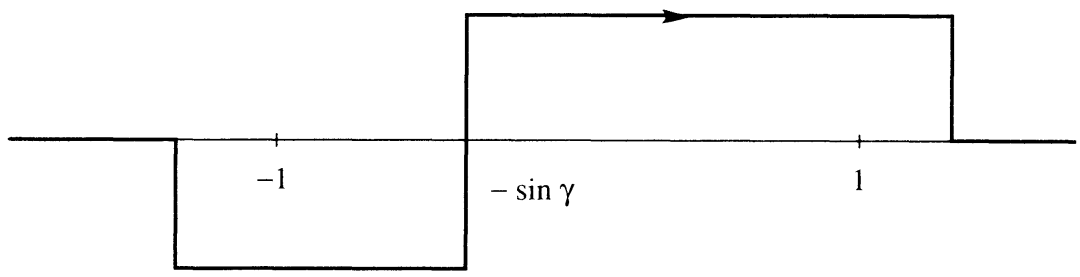

FIGURE 10

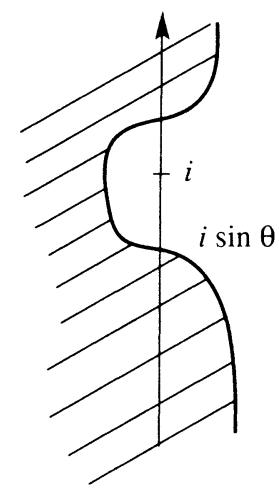

FIGURE 11

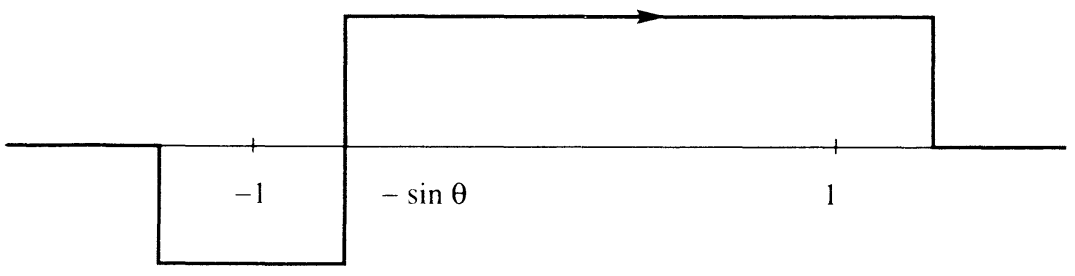

FigURE 12

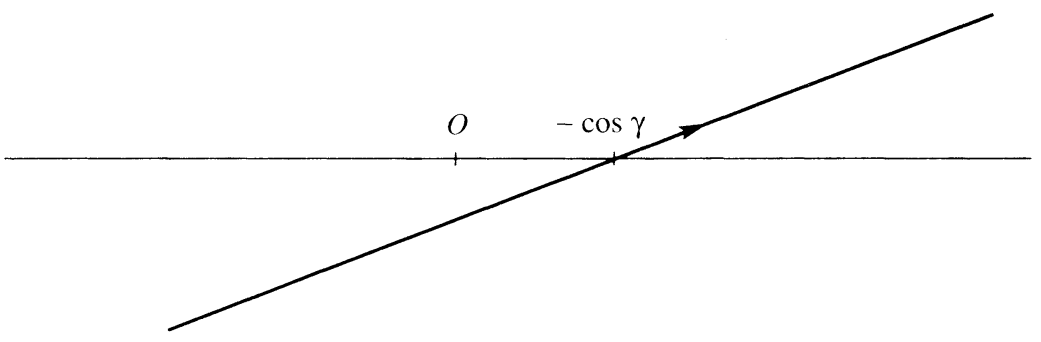

Figure 13

i.e., $(n+1) \gamma \geq \pi / 2$, alors on peut déformer sur le contour $\Gamma$ et conclure que $S(1)^{n+1} R_{-\alpha}$ envoie $L$ dans $H_{0}$.

Si maintenant $\gamma \geq \pi / 2$, on déforme $\mathbb{R} e^{i \alpha}$ sur un contour passant par $-\cos \gamma$ (voir la Figure 13) et on conclut immédiatement que $S(1) R_{-\alpha}: L \rightarrow H_{0}$. 


\section{Fin DE LA DÉMONSTRATION}

D’après la $\S 5$ et la Proposition A.3 de l'Appendice A, le système (4.115) se résout suivant

$$
\left(G_{+}, G_{-}\right)=\mathscr{B}\left(L_{+}, L_{-}\right),
$$

où $\mathscr{B}$ est un opérateur pseudo-différentiel à valeurs opérateurs dans la chaîne $H_{\sigma}^{+} \oplus H_{\sigma}^{-}$, de symbole principal $(I-\widetilde{A}(\tau, \lambda))^{-1}$, où $\widetilde{A}(\tau, \lambda)$ a été défini en (4.116). En appliquant $J_{\rho}^{-1}$, on en déduit que

$$
\left(F_{+}, F_{-}\right)=\mathscr{A}\left(l_{2}^{+}+l_{3}^{+}, l_{2}^{-}+l_{3}^{-}\right)
$$

modulo un élément négligeable de $H_{\varphi_{0}}\left(W_{\kappa / 2}, H_{0}^{+} \oplus H_{0}^{-}\right)$, avec $\mathscr{A}=J_{\rho}^{-1} \mathscr{B} J_{\rho}$, dont le symbole principal est, compte tenu de (4.116), (4.118), (5.1):

$$
\begin{aligned}
\sigma_{0}(\mathscr{A})(\tau) & =M(I-A(\tau))^{-1} M^{-1}, \\
M & =\left(\begin{array}{cc}
M_{+} & 0 \\
0 & M_{-}
\end{array}\right) .
\end{aligned}
$$

D'après la Proposition A.1 de l'Appendice A avec $\varphi=\varphi_{1}$, les estimations sur $l_{2}^{ \pm}, l_{3}^{ \pm}$, on conclut que

$$
F_{ \pm} \in H_{\varphi_{1}}\left(W_{\kappa / 2}, H_{0}^{+} \oplus H_{0}^{-}\right) .
$$

Une telle estimation signifie exactement (voir [L1]) que $f_{ \pm}$est conormale analytique par rapport à $\{t=x=0\}$ près de $\tau>0$, en dehors d'un voisinage d'ordre $\varepsilon_{2}$ de la direction $\xi+\tau / \cos \gamma_{ \pm}=0$. Posons alors

$$
u_{d}(t, x, y)=-\square^{-1}\left(f_{+} \delta_{+}+f_{-} \delta_{-}\right) \text {. }
$$

D'après (3.108), on a, près de $t=0$,

$$
u=u_{e}+u_{d}
$$

D'autre part, en écrivant la paramétrix de $\square$ dans les espaces $H_{\varphi}$ (voir [S2]) et en utilisant l'action des transformations canoniques quantifiées sur le second micro-support à croissance relativement à une lagrangienne (voir [L1]) on conclut que $u_{d}$ est conormale analytique par rapport à $\left\{t=\sqrt{x^{2}+y^{2}}\right\}$, près de $t>0$, et, si $\varepsilon_{2}$ a été choisi assez petit par rapport à $\varepsilon$, pour $(t, x, y) \in \Omega^{\varepsilon}$, et $t<\varepsilon_{1}$, pour $\varepsilon_{1}$ assez petit.

En reprenant la démonstration en microlocalisant près de $\tau=-1, t=0$, on démontre le même résultat près de $\tau<0$. Ceci entraîne qu'il existe un symbole analytique $\sigma_{d}=\sigma_{d}(x, y, \tau)$ tel que, modulo une fonction analytique, on ait, dans $\Omega^{\varepsilon} \cap\left\{0<t<\varepsilon_{1}\right\}$,

$$
u_{d}(t, x, y)=\int_{-\infty}^{\infty} e^{i \tau\left(t-\sqrt{x^{2}+y^{2}}\right)} \sigma_{d}(x, y, \tau) \frac{d \tau}{2 \pi},
$$

où, compte tenu de la vitesse finie de propagation,

$$
\sup _{s \geq 0} \int_{-\infty}^{\infty}\left(1+\tau^{2}\right)^{-D}\left|\sigma_{d}(x, y, \tau-i s)\right|^{2} d \tau<+\infty,
$$


pour un certain réel $D$. Ceci prouve le point (1.16) du Théorème 1.1 et le fait que $u_{d}$ est conormale analytique sur $\Sigma_{d}$. Pour vérifier les points (1.17) à (1.20) il suffit à présent d'identifier $\sigma_{d}$.

Fixons $\left(x_{0}, y_{0}\right)$, avec $\left(\sqrt{x_{0}^{2}+y_{0}^{2}}, x_{0}, y_{0}\right) \in \Omega^{\varepsilon} \cap\left\{t<\varepsilon_{1}\right\}, x_{0}=t_{0} \cos \theta_{0}$, $y_{0}=t_{0} \sin \theta_{0}, t_{0}=\sqrt{x_{0}^{2}+y_{0}^{2}}$.

Soit $\sigma_{+}(\tau, \xi)$ un symbole analytique de degré $d$, au sens $L^{2}$, défini au voisinage conique de $\left(\tau_{0}, \xi_{0}\right), \tau_{0}=1, \xi_{0}=-\cos \left(\theta_{0}-\gamma_{+}\right) \tau_{0} / \cos \gamma_{+},\left(\theta_{0}-\gamma_{+}\right) \neq$ 0 tel que microlocalement près de $t=x=0, \tau=\tau_{0}, \xi=\xi_{0}$ on ait

$$
f_{+}=(2 \pi)^{-2} \int e^{i \tau t+i x \xi} \sigma_{+} d \tau d \xi .
$$

Alors près de $\left(t_{0}, x_{0}, y_{0}, \tau_{0}, \xi_{0}=-\cos \theta_{0}, \eta_{0}=-\sin \theta_{0}\right)$ on obtient microlocalement par un calcul classique sur les paramétrix des opérateurs à caractéristiques simples

$$
g_{+}=-\square^{-1}\left(f_{+} \delta_{+}\right)=\int e^{i \tau\left(t-\sqrt{x^{2}+y^{2}}\right)} \sigma_{d,+}(x, y, \tau) \frac{d \tau}{2 \pi}
$$

avec, $r$ désignant un symbole de degré $\leq d-3 / 2$,

$$
\sigma_{d,+}(x, y, \tau)=\frac{e^{3 i \pi / 4}}{\sqrt{8 \pi \tau}}\left(x^{2}+y^{2}\right)^{-1 / 4} \sigma_{+}\left(\tau,-\tau \frac{x+a y}{\sqrt{x^{2}+y^{2}}}\right)+r
$$

et d'autre part, près de $w=-i \tau_{0}, z=i \tau_{0}\left(x_{0}+a y_{0}\right) / \sqrt{x_{0}^{2}+y_{0}^{2}}$,

$$
\begin{aligned}
F_{+}(w, z, \lambda)= & \left.e^{-\lambda w^{2} / 2} \sum_{\substack{m \leq \lambda / C \\
n \leq \lambda / C}}(2 \lambda)^{-(n+m)} \frac{\partial_{\tau}^{2 n}}{n !} \frac{\partial_{\xi}^{2 m}}{m !} \sigma_{+}(\lambda \tau, \lambda \xi)\right|_{\tau=i w, \xi=i z} \\
& +\mathscr{O}\left(e^{\lambda \varphi_{0}(w)-\lambda / D}\right) .
\end{aligned}
$$

En comparant (6.12) et (6.13), on obtient donc au premier ordre

$$
\sigma_{d,+}(x, y, \tau) \sim \frac{e^{3 i \pi / 4}}{\sqrt{8 \pi \tau}}\left(x^{2}+y^{2}\right)^{-1 / 4} e^{-\tau / 2} F_{+}\left(-i, i \frac{x+a y}{\sqrt{x^{2}+y^{2}}}, \tau\right)
$$

et, en utilisant les formules (6.2), (6.3), (6.4) et la Remarque A.2 de l'Appendice A, on a, au premier ordre

$$
\left(F_{+}, F_{-}\right)(-i, \tau) \sim M(1-A(1))^{-1} M^{-1}\left(l_{2}^{+}+l_{3}^{+}, l_{2}^{-}+l_{3}^{-}\right)(-i, \tau),
$$

et, compte tenu de (4.120), (4.121), (4.122),

$$
\left(l_{2}^{ \pm}+l_{3}^{ \pm}\right)(-i, z, \tau) \sim \sigma_{i}(0,0, \tau) m_{ \pm}(-i, z) e^{\tau / 2} .
$$

On en déduit que le terme principal de $\sigma_{d,+}$ pour $\tau>0$ est, en posant $x / \sqrt{x^{2}+y^{2}}=\cos \theta, y / \sqrt{x^{2}+y^{2}}=\sin \theta$,

$$
\sigma_{d,+}^{0}(x, y, \tau)=\left(\tau \sqrt{x^{2}+y^{2}}\right)^{-1 / 2} K_{+}\left(\cos \left(\theta-\gamma_{+}\right)\right) \sigma_{i}(0,0, \tau),
$$


où $K_{+}$est une fonction spéciale que nous caractérisons ci-dessous. De même la contribution principale de $-\square^{-1}\left(f_{-} \delta_{-}\right)$à $\sigma_{d}(x, y, \tau)$ est donnée par

$$
\sigma_{d,-}^{0}(x, y, \tau)=\left(\tau \sqrt{x^{2}+y^{2}}\right)^{-1 / 2} K_{-}\left(\cos \left(\theta-\gamma_{-}\right)\right) \sigma_{i}(0,0, \tau) .
$$

Enfin les fonctions $K_{+}, K_{-}$sont données par les formules suivantes:

$$
\left(K_{+}, K_{-}\right)=\frac{e^{3 i \pi / 4}}{\sqrt{8 \pi}}(1-A(1))^{-1}\left(\tilde{m}_{+}, \tilde{m}_{-}\right),
$$

où $1-A(1)$ a été inversé au paragraphe 5 , et

$$
\tilde{m}_{ \pm}(z)= \pm \frac{1}{2 \pi} \int_{\mathscr{C}_{ \pm}} \frac{\cos \gamma_{ \pm} \mp\left(\xi \sin \gamma_{ \pm}\right) / \xi_{0}}{z+i\left(\xi \cos \gamma_{ \pm} \pm \xi_{0} \sin \gamma_{ \pm}\right)} \frac{2 \sin \gamma_{\mp} d \xi}{\xi \cos \gamma_{\mp} \pm \xi_{0} \sin \gamma_{\mp}+\cos \gamma_{\mp}}
$$

où $\mathscr{C}_{+}=\mathscr{C}(l, a), \mathscr{C}_{-}=\mathscr{C}(l,-b)$, ont été définis en $(4.91)$.

Pour $\tau<0$, les formules (6.17), (6.18) sont les mêmes, puisque $\sigma_{d}$ est holomorphe dans $\operatorname{Im} \tau<0$. Ceci achève la démonstration du Théorème 1.1 .

Montrons à présent le Théorème 1.2. Soit $q \in T^{*} \Omega \cap W F_{a}(u)$. On peut supposer $q=\left(t_{0}, x_{0}, y_{0} ; \tau_{0}, \xi_{0}, \eta_{0}\right)$ avec $t_{0}>0$ (car $u=u_{i}$ dans $t<0$ ) et $\tau_{0}>0$ (quitte à changer $u$ en $\bar{u}$ ). Soit $\varepsilon>0$ petit. D'après le théorème de propagation de J. Sjöstrand ([S1]), il existe un demi-rayon maximal $\rho$, tourné vers les temps négatifs, défini sur $I=\left[0, s_{0}[\right.$, contenu dans $t>-\varepsilon$, avec $\rho(s) \notin T^{*} L$, et contenu dans le front d'onde analytique jusqu'au bord de $u$. Si $t\left(s_{0}\right)=-\varepsilon$ on a bien $q \in S$ par définition de $S$. Sinon on a nécessairement $\rho\left(s_{0}\right) \in T^{*} L$ et il s'agit de prouver $t\left(s_{0}\right)=0$. Comme on a $u=u_{e}+u_{d}$, $u_{d}=-\square^{-1}\left(f_{+} \delta_{+}+f_{-} \delta_{-}\right)$près de $t=0, u_{e}$ étant défini par (1.12), il suffit de vérifier qu'on a, pour tout $\xi,\left(t, \tau_{0}, 0, \xi\right) \notin W F_{a}\left(f_{ \pm}\right)$pour $t \neq 0$. Or d'après ce qui précède, cela est vrai pour $t \neq 0$ petit et $\left|\xi+\tau_{0} / \cos \gamma_{ \pm}\right|>\varepsilon_{2}$. Comme $f_{ \pm}$est à support dans $x \geq 0$, le résultat est donc conséquence du théorème du Watermelon de M. Kashiwara.

\section{ApPendice A. CAlcul pseudo-diffÉrentiel COMPlexe À VALEURS OPÉRATEURS DANS UNE CHAÎNE D'ESPACES DE BANACH}

Soit $\left(H_{\sigma}\right)$ une chaîne d'espaces de Banach au sens de [O, N, BG]. On note ||$_{\sigma}$ la norme sur $H_{\sigma},||_{\sigma^{\prime}, \sigma}$ la norme d'opérateur de $H_{\sigma}$ dans $H_{\sigma^{\prime}}$. Si $U$ est un ouvert de $T^{*} \mathbb{C}^{d}$, un symbole à valeurs opérateurs sur $U$ et sur la chaîne $\left(H_{\sigma}\right)$ est une série formelle

$$
a(w, \tau, \lambda)=\sum_{n=0}^{\infty} a_{n}(w, \tau, \lambda)(i \lambda)^{-n}
$$

où, pour tout $n, a_{n}$ est une fonction holomorphe de $(w, \tau) \in U$ à valeurs dans les opérateurs bornés de $H_{\sigma}$ dans $H_{\sigma^{\prime}}$ pour tout $\sigma^{\prime}<\sigma$, telle que

$$
\sup _{(w, \tau) \in U}\left|a_{n}(w, \tau, \lambda)\right|_{\sigma^{\prime}, \sigma} \leq A B^{n}\left(\frac{n}{\sigma-\sigma^{\prime}}\right)^{n}
$$

avec $A, B$ indépendants de $\lambda$. 
On quantifie de tels symboles de la même manière qu'à la §3.1. Soient $W$ un ouvert de $\mathbb{C}^{d}, \tau_{0} \in \mathbb{C}^{d}$ et $R_{0}>0$ tels que $W \times B\left(\tau_{0}, R_{0}\right) \subset U$. Si $\varphi \in C^{1}(W)$ et $m \in \mathbb{R}$, on désigne par $H_{\varphi}^{m}\left(W, H_{\sigma}\right)$ l'espace de Sjöstrand des fonctions holomorphes $f$ sur $W$, à valeurs dans $H_{\sigma}$, et dépendant d'un paramètre $\lambda \geq 1$ de sorte que

$$
\forall w \in W, \quad|f(w, \lambda)|_{\sigma} \leq C \lambda^{m} e^{\lambda \varphi(w)} .
$$

On note $H_{\varphi}\left(W, H_{\sigma}\right)=\bigcup_{m} H_{\varphi}^{m}\left(W, H_{\sigma}\right)$.

On suppose enfin que

$$
\forall w \in W, \quad\left\|\frac{2}{i} \frac{\partial \varphi}{\partial w}(w)-\tau_{0}\right\| \leq D<R_{0} .
$$

Définition-Proposition A.1. Soit a un symbole à valeurs opérateurs sur $\left(H_{\sigma}\right)$, et soient $C_{1}, C_{2}$ deux constantes positives. Pour $\sigma^{\prime}<\sigma$, on pose

$$
\begin{aligned}
\mathrm{Op}_{\sigma^{\prime}, \sigma}(a)\left(\tau_{0}, C_{1}, C_{2}\right) \\
\quad=\sum_{n \leq \lambda\left(\sigma-\sigma^{\prime}\right) / C_{1}|\alpha| \leq \lambda / C_{2}}(i \lambda)^{-n} \frac{1}{\alpha !} g_{\tau}^{(x} a_{n}\left(w, \tau_{0}, \lambda\right)\left(\frac{1}{i \lambda} \partial_{w}-\tau_{0}\right)^{\alpha} .
\end{aligned}
$$

Soit $W^{\prime} \Subset W$. Alors, si $C_{1} \geq 2 B, C_{2} \geq\left(D \operatorname{dist}\left(W^{\prime}, W^{c}\right)\right)^{-1}$, l'opérateur cidessus envoie $H_{\varphi}^{m}\left(W, H_{\sigma}\right)$ dans $H_{\varphi}^{m}\left(W^{\prime}, H_{\sigma^{\prime}}\right)$. De plus, si $C_{1}^{\prime}$ et $C_{2}^{\prime}$ est un autre couple de constantes satisfaisant les inégalités ci-dessus, il existe $\varepsilon>0$ tel que lopérateur

$$
\mathrm{Op}_{\sigma^{\prime}, \sigma}(a)\left(\tau_{0}, C_{1}, C_{2}\right)-\mathrm{Op}_{\sigma^{\prime}, \sigma}(a)\left(\tau_{0}, C_{1}^{\prime}, C_{2}^{\prime}\right)
$$

envoie $H_{\varphi}^{m}\left(W, H_{\sigma}\right)$ dans $H_{\varphi-\varepsilon}\left(W^{\prime}, H_{\sigma^{\prime}}\right)$.

Remarque A.1. En vertu du théorème de la phase stationnaire, la quantification ci-dessus peut encore s'écrire, modulo un terme dans $H_{\varphi-\varepsilon}$,

$$
\begin{aligned}
& \mathrm{Op}_{\sigma^{\prime}, \sigma}(a)\left(\tau_{0}, C_{1}, C_{2}\right) f(w, \lambda) \\
& \quad \equiv\left(\frac{\lambda}{2 \pi}\right)^{d} \iint_{\Sigma_{C_{2}}} e^{i \lambda\left(w-w^{\prime}\right) \tau} a_{C_{1}}(w, \tau, \lambda) f\left(w^{\prime}, \lambda\right) d w^{\prime} \wedge d \tau,
\end{aligned}
$$

où $a_{C_{1}}=\sum_{n \leq\left(\sigma-\sigma^{\prime}\right) \lambda / c_{1}} a_{n}(i \lambda)^{-n}$, et où $\Sigma_{C_{2}}$ est un "bon contour" de $\mathbb{C}^{d} \times \mathbb{C}^{d}$ au sens de [S2].

Remarque A.2. Dans le cas où $\varphi(w)=\varphi_{1}(w)=\left((\operatorname{Im} w)^{2}-(\operatorname{Re} w)^{2}\right) / 2$, une fonction $f$ de $H_{\varphi_{1}}^{m}$ peut encore s'écrire $e^{-\lambda w^{2} / 2} \tilde{f}$, où $\tilde{f} \in H_{0}^{m}$. On peut alors identifier aisément le terme prépondérant de $\mathrm{Op}(a) f$. Précisément, on a

$$
\mathrm{Op}_{\sigma^{\prime}, \sigma}(a)\left(\tau_{0}, C_{1}, C_{2}\right)=a_{0}(w, i w, \lambda)+R,
$$

où $R: H_{\varphi_{1}}^{m}\left(W, H_{\sigma}\right) \rightarrow H_{\varphi_{1}}^{m+1}\left(W^{\prime}, H_{\sigma^{\prime}}\right)$. 
Pour vérifier la formule ci-dessus, on évacue tout d'abord la contribution des $a_{n}$ pour $n \geq 1$ dans un terme de type $R$ grâce à la Proposition A.1. Ensuite, il suffit d'utiliser la Remarque A.1 et la méthode du col.

On dispose par ailleurs du calcul symbolique habituel:

Proposition A.2. Soient $a$ et $b$ deux symboles sur $U$ à valeurs opérateurs sur $\left(H_{\sigma}\right)$. On définit $a \circ b$ par

$$
(a \circ b)_{n}(w, \tau, \lambda)=\sum_{p+q+|\alpha|=n} \frac{1}{\alpha !} \partial_{\tau}^{\alpha} a_{p}(w, \tau, \lambda) \partial_{w}^{\alpha} b_{q}(w, \tau, \lambda)
$$

Alors $a \circ b$ est un symbole sur tout $U_{1} \Subset U$, et il existe des constantes $C_{1}^{0}, C_{2}^{0}$ telles que, pour tous $C_{1} \geq C_{1}^{0}, C_{2} \geq C_{2}^{0}, \sigma^{\prime}<\sigma^{\prime \prime}<\sigma$, lopérateur

$$
\mathrm{Op}_{\sigma^{\prime}, \sigma}(a \circ b)\left(\tau_{0}, C_{1}, C_{2}\right)-\mathrm{Op}_{\sigma^{\prime}, \sigma^{\prime \prime}}(a)\left(\tau_{0}, C_{1}, C_{2}\right) \mathrm{Op}_{\sigma^{\prime \prime}, \sigma}(b)\left(\tau_{0}, C_{1}, C_{2}\right)
$$

envoie $H_{\varphi}^{m}\left(W, H_{\sigma}\right)$ dans $H_{\varphi-\varepsilon}\left(W^{\prime}, H_{\sigma^{\prime}}\right)$ avec $\varepsilon>0$.

Les démonstrations des Propositions A.1 et A.2 sont analogues à celles des Lemmes 3.1 et 3.3 et sont laissées au lecteur. On remarquera que la constante $\varepsilon>0$ qui décrit la décroissance des restes est de la forme $\varepsilon=\left(\sigma-\sigma^{\prime}\right) \varepsilon_{0}$ avec $\varepsilon_{0}>0$ indépendant de $\sigma, \sigma^{\prime}$.

Signalons enfin le théorème d'inversibilité des symboles elliptiques. Rappelons qu'une chaîne d'opérateurs $a=a_{\sigma^{\prime}, \sigma}: H_{\sigma} \rightarrow H_{\sigma^{\prime}}, \sigma^{\prime}<\sigma$, est dite inversible s'il existe une chaîne $b=b_{\sigma^{\prime}, \sigma}: H_{\sigma} \rightarrow H_{\sigma^{\prime}}, \sigma^{\prime}<\sigma$, telle que, pour tous $\sigma^{\prime}<\sigma^{\prime \prime}<\sigma$, on ait

$$
b_{\sigma^{\prime}, \sigma^{\prime \prime}} a_{\sigma^{\prime \prime}, \sigma}=a_{\sigma^{\prime}, \sigma^{\prime \prime}} b_{\sigma^{\prime \prime}, \sigma}=I_{\sigma^{\prime}, \sigma}
$$

où $I_{\sigma^{\prime}, \sigma}$ désigne l'injection canonique. La chaîne $b$ est alors unique, notée $a^{-1}$.

Proposition A.3. Soit a un symbole sur $U$ à valeurs opérateurs sur $\left(H_{\sigma}\right)$. On suppose que, pour tous $(w, \tau) \in U$ et $\lambda \geq 1, a_{0}(w, \tau, \lambda)$ est inversible et que

$$
\sup _{\sigma^{\prime}<\sigma(w, \tau) \in U} \sup _{\lambda} \sup _{\lambda}\left|a_{0}^{-1}(w, \tau, \lambda)\right|_{\sigma^{\prime}, \sigma}<x
$$

Alors, pour tout $U_{1} \Subset U$, il existe un unique symbole $b$ sur $U_{1}$ tel que

$$
b \circ a=a \circ b=1 \text {. }
$$

De plus $b_{0}=a_{0}^{-1}$.

La démonstration de la Proposition A.3 est une adaptation simple de celle de Sjöstrand [S2, Chapitre 1]. 


\section{APPENDice B. DÉveloppements ASymptotiques}

Preuve de la Proposition 4.1, §4.3. (B.1)

D'abord on écrit $I=\lim _{\nu \rightarrow 0^{+}} I^{\nu}$, dans $\operatorname{Im} \tau<0$, avec

$$
\begin{aligned}
I^{\nu}=\int_{0}^{\infty} e^{\lambda x z-\lambda x^{2} / 2} & \left\{\psi_{2}(x) \frac{\lambda}{2 \pi} \int_{\mathbb{R}} d x^{\prime} \int_{\mathscr{G}_{1}} d \xi e^{i \lambda\left(x-x^{\prime}\right) \xi-\nu \xi^{2}}\right. \\
& \left.\times \psi(x) \psi\left(x^{\prime}\right) \sigma\left(x, x^{\prime}, \tau, \xi\right) T_{0} f_{+}\left(t-i \tau, x^{\prime}, \lambda\right)\right\} d x .
\end{aligned}
$$

(On introduit d'abord le facteur de convergence $e^{-\nu \xi^{2}}$, puis on déforme l'intégrale en $\xi \in \mathbb{R}$ sur $\mathscr{C}_{1}$.) D'après le Lemme 4.4 , (B.1) entraîne que $I^{\nu}$ est défini pour $(t, \tau) \in X_{\kappa}, z \in U$. Ensuite on a

$$
I^{\nu}=\sum_{n \leq N} I_{n}^{\nu}+I_{R, N}^{\nu}
$$

en utilisant le développement de Taylor de $\sigma$

$$
\sigma=\sum_{n \leq N} \frac{\sigma^{(n)}(0)}{n !} x^{n}+\frac{x^{N+1}}{N !} \int_{0}^{1} \sigma^{(N+1)}(s x)(1-s)^{N} d s .
$$

Alors on a, avec $N=E\left(\lambda / C_{1}^{0}\right), C_{1}^{0}=e D_{1} / a^{\prime}$,

$$
I_{R, N}=\lim _{\nu \rightarrow 0^{+}} I_{R, N}^{\nu} \text { est négligeable dans } H\left(\varphi_{0}, X_{\kappa}, U\right) .
$$

En effet, par intégration par parties en $x$, on a

$$
\begin{aligned}
\lim _{\nu \rightarrow 0^{+}} I_{R, N}^{\nu}= & \frac{\lambda}{2 \pi} \int_{0}^{\infty} d x \iint \frac{e^{\lambda(x z+i x \xi)}}{(z+i \xi)^{3}}\left(\frac{-1}{\lambda} \partial_{x}\right)^{3} \\
& \times\left[e^{-\lambda x^{2} / 2-i \lambda x^{\prime} \xi} \psi(x) \psi\left(x^{\prime}\right) \frac{x^{N+1}}{N !} \int_{0}^{1} \sigma^{(N+1)}(s x)(1-s)^{N} d s\right. \\
& \left.\quad \times T_{0} f_{+}\left(t-i \tau, x^{\prime}, \lambda\right)\right] d x^{\prime} d \xi .
\end{aligned}
$$

Or pour $z \in U, \xi \in \mathscr{C}_{1}$, on a $\operatorname{Re} z-\operatorname{Im} \xi \leq-A \sin \varepsilon+a^{\prime \prime} \leq-4+1+4 c t_{0} \leq-2$ (car $t_{0} \ll 1$ ) et d'après le Lemme 4.4 , pour $x \in \operatorname{support}(\psi),\left|\sigma^{(k)}(x)\right| \leq$ $k !\left(D_{1} / a^{\prime}\right)^{k+1}$. Comme $\int_{\mathscr{C}_{1}} d \xi /|z+i \xi|^{3} \leq D /(1+|z|)^{2}$ pour $z \in U$, on obtient

$$
\left|I_{R, N}\right| \leq D \lambda^{D} \frac{\left\|T_{0} f_{+}(t-i \tau, \cdot, \lambda)\right\|_{L^{2}}}{(1+|z|)^{2}} e^{4 \lambda c t_{0} a^{\prime}}\left(\frac{D_{1}}{a^{\prime}}\right)^{N+4} \frac{N !}{\lambda^{N}}
$$

et on a $\left(D_{1} N / a^{\prime} \lambda\right)^{N} \leq e^{-N} \leq e e^{-\lambda a^{\prime} / e D_{1}}$, et $1 / e D_{1}>4 c t_{0}$ car $t_{0} \ll 1$.

On écrit

$$
I_{n}^{\nu}=\int_{0}^{\infty} d x \int_{\mathscr{E}_{1}} d \xi e^{\lambda x z-\lambda x^{2} / 2+i \lambda x \xi} \psi(x) J_{n}(\xi, t, \tau, \lambda) \frac{\lambda}{2 \pi} e^{-\nu \xi^{2}} x^{n}
$$




$$
J_{n}=\int d x^{\prime} e^{-i \lambda x^{\prime} \xi} \psi\left(x^{\prime}\right) \frac{\partial_{x}^{n} \sigma\left(0, x^{\prime}, \tau, \xi\right)}{n !} T_{0} f_{+}\left(t-i \tau, x^{\prime}, \lambda\right)
$$

et on utilisera le lemme suivant.

Lemme B.1. Soit $\left\{J_{n}^{1}(\xi, t, \tau, \lambda)\right\}_{n \geq 0}$ une famille de fonctions holomoprhes pour $(t, \tau) \in X_{\kappa}, L^{2}$ en $\xi$ sur $\mathscr{C}_{1}$, telle que

$$
\exists C>0, \quad\left\|J_{n}^{1}\right\|_{L^{2}\left(\mathscr{C}_{1}\right)} \leq C e^{-\lambda / C}\left(\frac{D_{1}}{a^{\prime}}\right)^{n+1} e^{\lambda \varphi_{0}(t-i \tau)} .
$$

Alors

$$
\sum_{n \leq \lambda / C_{1}^{0}} \int_{0}^{\infty} d x \int_{\mathscr{C}_{1}} d \xi e^{\lambda x z-\lambda x^{2} / 2+i \lambda x \xi} \psi(x) x^{n} J_{n}^{1} e^{-\nu \xi^{2}}
$$

est (uniformément en $\nu$ ), négligeable dans $H\left(\varphi_{0}, X_{\kappa}, U\right)$.

Preuve. Posons $\Theta(z, \lambda)=\int_{0}^{\infty} e^{\lambda x z-\lambda x^{2} / 2} \psi(x) d x$. En intégrant par parties une fois en $x$, on obtient pout $\operatorname{Re} z \leq-1,|\Theta(z, \lambda)| \leq D / \lambda|z|$ donc pour $\operatorname{Re} z \leq$ $-2,\left|\Theta^{(j)}(z, \lambda)\right| \leq D j ! / \lambda|z|$. Alors

$$
\int_{0}^{\infty} d x e^{\lambda x z-\lambda x^{2} / 2+i \lambda x \xi} \psi(x) x^{n} d x=\left(\frac{\partial_{z}}{\lambda}\right)^{n} \Theta(z+i \xi),
$$

est estimé pour $z \in U, \xi \in \mathscr{C}_{1}$ par $D n ! \lambda^{-(n+1)} /|z+i \xi|$. Donc l'opérateur $g \mapsto$ $\int_{0}^{\infty} d x \int_{\mathscr{C}_{1}} e^{\lambda x z-\lambda x^{2} / 2+i \lambda x \xi} g(\xi) \psi(x) x^{n} d \xi$ est borné par $D n ! \lambda^{-(n+1)}$ de $L^{2}\left(\mathscr{C}_{1}\right)$ dans $H_{U}$ et on a $\left(D_{1} / a^{\prime}\right)^{n} n ! \lambda^{-n} \leq\left(n D_{1} / a^{\prime} \lambda\right)^{n} \leq e^{-n}$, car $n \leq \lambda / C_{1}^{0}=$ $\lambda a^{\prime} / e D_{1}$.

D'après la formule d'inversion (4.5), on a, en intégrant par parties en $x^{\prime}$,

$$
\begin{aligned}
& J_{n}(\xi, t, \tau, \lambda)=\iint_{\mathbb{R}^{2}} d x^{\prime} d \alpha j_{n}\left(\xi, t, \tau, \lambda ; x^{\prime}, \alpha\right), \\
j_{n}= & \frac{\lambda}{2 \pi} \frac{e^{i \lambda \alpha x^{\prime}}}{\left(1+\alpha^{2}\right)}\left(1-\frac{1}{\lambda^{2}} \Delta_{x^{\prime}}\right) \\
& \times\left[e^{-\lambda x^{\prime 2} / 2} \psi\left(x^{\prime}\right) \frac{d_{x}^{n} \sigma\left(0, x^{\prime}, \tau, \xi\right)}{n !} F_{+}\left(t-i \tau, x^{\prime}-i \xi-i \alpha, \lambda\right)\right] .
\end{aligned}
$$

Pour étudier $I_{n}^{\nu}$, on décompose $\mathscr{C}_{1}$ en deux morceaux $\mathscr{C}_{1}=\mathscr{C}_{1}^{-} \cup \mathscr{C}_{1}^{+}$avec

$$
\mathscr{C}_{1}^{-}=\left\{\xi \in \mathscr{C}_{1} ;\left|\operatorname{Re} \xi-\frac{1}{\cos \gamma_{+}}\right| \geq 1+D_{1} t_{0}\right\} \text {. }
$$

Pour $\xi \in \mathscr{C}_{1}^{-}, x^{\prime} \in \operatorname{support}(\psi)$, on a $\operatorname{Re}\left(x^{\prime}-i \xi-i \alpha\right) \leq 4 c t_{0}-a^{\prime \prime} \leq-1$, donc d'après (4.12)

$$
\left\|j_{n}\right\|_{L^{2}\left(\mathscr{C}_{-}^{-}\right)} \leq D \lambda^{D} e^{-\lambda x^{\prime 2} / 2}\left(\frac{D_{1}}{a^{\prime}}\right)^{n+1} e^{\lambda \varphi_{0}(t-i \tau)} \frac{\left\|f_{+}\right\|_{L^{2}}}{1+\alpha^{2}}
$$

pour $(t, \tau) \in X_{\kappa}, x^{\prime} \in \operatorname{support}(\psi), \alpha \in \mathbb{R}$. 
Soient $g \in C_{0}^{\infty}(]-2 c t_{0},+2 c t_{0}[), 0 \leq g \leq 1, g \equiv 1$ sur $\left[-c t_{0}, c t_{0}\right]$ et $\Sigma$ le contour

(B.15) $\Sigma=\left\{\left(\alpha, x^{\prime}\right) ; \alpha \in \mathbb{R}, x^{\prime}=y^{\prime}+i g\left(y^{\prime}\right) \frac{\alpha}{\left(1+\alpha^{2}\right)^{1 / 2}} \frac{a^{\prime}}{D_{1}} ; y^{\prime} \in \operatorname{support} \psi\right\}$.

D'après (4.12) et (B.12), on a

(B.16)

$$
\left\|j_{n}\right\|_{L^{2}\left(\mathscr{C}_{1}^{-}\right)} \leq D \lambda^{D} e^{-(\lambda / 2)\left[y^{\prime 2}+\left(a^{\prime} / D_{1}\right) \alpha^{2} g\left(y^{\prime}\right) /\left(1+\alpha^{2}\right)^{1 / 2}\right]}\left(\frac{D_{1}}{a^{\prime}}\right)^{n+1} e^{\lambda \varphi_{0}(t-i \tau)} \frac{\left\|f_{+}\right\|_{L^{2}}}{1+\alpha^{2}}
$$

pour $(t, \tau) \in X,\left(\alpha, x^{\prime}\right) \in \Sigma$. Si on se donne $r>0$, et si on pose

$$
\Sigma_{0}=\Sigma \cap\left\{\left|y^{\prime}\right|+|\alpha|<r\right\}, \quad \Sigma_{1}=\Sigma \backslash \Sigma_{0}
$$

on a pour $\xi \in \mathscr{C}_{1}^{-}$

$$
J_{n}=\widetilde{J}_{n}^{0}+\widetilde{J}_{n}^{1} ; \quad \widetilde{J}_{n}^{i}=\iint_{\Sigma_{i}} j_{n} d x^{\prime} d \alpha
$$

où $\widetilde{J}_{n}^{1}$ vérifie les estimées $($ B.9).

Maintenant, pour $\xi \in \mathscr{C}_{1}^{+}$, on a $\operatorname{Re}\left(x^{\prime}-i \xi-i \alpha\right) \leq x^{\prime}+a^{\prime}$ donc d'après (4.12) et (B.12)

$$
\left\|j_{n}\right\|_{L^{2}\left(\mathscr{C}_{1}^{+}\right)} \leq D \lambda^{D} \frac{\left\|f_{+}\right\|_{L^{2}}}{1+\alpha^{2}}\left(\frac{D_{1}}{a^{\prime}}\right)^{n+1} e^{-\lambda x^{\prime 2} / 2+\lambda \varphi_{0}(t-i \tau)+\lambda \Phi_{\delta}\left(x^{\prime}+a^{\prime}\right)}
$$

pour $(t, \tau) \in X_{\kappa}, x, \alpha$ réels. Déformons à nouveau l'intégrale qui définit $J_{n}$ sur le contour $\Sigma((\mathrm{B} .15))$. On obtient pour $\xi \in \mathscr{C}_{1}^{+},\left(x^{\prime}, \alpha\right) \in \Sigma,(t, \tau) \in X$

$$
\begin{aligned}
& \left|j_{n}\right| \leq D \lambda^{D} \frac{\left\|f_{+}\right\|_{L^{2}}}{1+\alpha^{2}}\left(\frac{D_{1}}{a^{\prime}}\right)^{n+1} e^{\lambda \varphi_{0}(t-i \tau)} e^{\lambda G}, \\
G \leq & -g\left(y^{\prime}\right) \frac{\alpha^{2}}{\left(1+\alpha^{2}\right)^{1 / 2}} \frac{a^{\prime}}{2 D_{1}}-\frac{y^{\prime 2}}{2} \\
& +\Phi_{\delta}^{+}\left(y^{\prime}+\operatorname{Im} \xi+i\left(\alpha\left(\frac{g\left(y^{\prime}\right)}{\left(1+\alpha^{2}\right)^{1 / 2}} \frac{a^{\prime}}{D_{1}}-1\right)-\operatorname{Re} \xi\right)\right)
\end{aligned}
$$

d'après (4.14). Vérifions alors qu'on a $G<0$ en dehors de $y^{\prime}=\alpha=0$.

Si $y^{\prime} \leq-c t_{0}$, on a

$$
y^{\prime}+\operatorname{Im} \xi \leq-c t_{0}+a^{\prime}<0,
$$

donc $\Phi_{\delta}^{+}=0$ et $G<0$.

Si $y^{\prime} \geq c t_{0}$, on a

$$
\begin{aligned}
-\frac{y^{\prime 2}}{2}+\Phi_{\delta}^{+} & \leq \frac{-y^{\prime 2}}{2}+\Phi_{\delta}\left(y^{\prime}+a^{\prime}\right) \\
& =\frac{y^{\prime 2}}{2}+\frac{\left(y^{\prime}+a^{\prime}\right)^{2}}{2}-\frac{1}{2\left(1+c^{2}\right)}\left(y^{\prime}+x^{\prime}-\delta\right)=h\left(y^{\prime}\right)
\end{aligned}
$$


de dérivée $h^{\prime}\left(y^{\prime}\right)=a^{\prime}-\left(y^{\prime}+a^{\prime}-\delta\right) /\left(1+c^{2}\right)<0$ car $\sup \left(a^{\prime}, \delta\right) \ll t_{0}$. (Rappelons que $\left.\delta=3 \kappa c=3 a^{\prime} c / D_{1}\right)$ et pour la même raison $h\left(c t_{0}\right)<0$ donc $G<0$.

Maintenant lorsque $\left|y^{\prime}\right| \leq c t_{0}$, on a $g\left(y^{\prime}\right) \equiv 1$. Choisissons un $\delta_{1}$ tel que $\sup \left(\delta, a^{\prime}\right) \ll \delta_{1} \ll d$. Pour $y^{\prime}+a^{\prime} \leq 0$, on a $\Phi_{\delta}^{+} \leq 0$ donc $G<0$. Pour $y^{\prime}+a^{\prime} \geq \delta_{1}$, on a $\Phi_{\delta}^{+} \leq \Phi_{\delta}$ donc $G \leq-\alpha^{2} a^{\prime} /\left(1+\alpha^{2}\right)^{1 / 2} 2 D_{1}+h\left(y^{\prime}\right)$ et $h\left(y^{\prime}\right)<0$ car $\delta_{1} \gg \sup \left(\delta, a^{\prime}\right)$.

Enfin, lorsque $y^{\prime}+a^{\prime} \in\left[0, \delta_{1}\right]$, on aura d'après (4.14) soit $\Phi_{\delta}^{+}=0$ donc $G<0$ pour $\left(y^{\prime}, \alpha\right) \neq(0,0)$, soit $\Phi_{\delta}^{+} \leq \Phi_{\delta}\left(y^{\prime}+a^{\prime}\right)$ et

$$
\left|\alpha\left(1-\frac{a^{\prime}}{D_{1}} \frac{1}{\left(1+\alpha^{2}\right)^{1 / 2}}\right)+\operatorname{Re} \xi+\frac{1}{\cos \gamma_{+}}\right| \leq \varepsilon_{0},
$$

donc $\alpha$ de l'ordre de $-2 / \cos \gamma_{+}$, donc

$$
G \leq-\frac{a^{\prime}}{D_{1} D}-\frac{y^{\prime 2}}{2}+\frac{\left(y^{\prime}+a^{\prime}\right)^{2}}{2}=-a^{\prime}\left[\frac{1}{D_{1} D}-y^{\prime}-\frac{a^{\prime}}{2}\right]<0
$$

si $\delta_{1}$ est assez petit, soit enfin $\Phi_{\delta}^{+} \leq \Phi_{\delta}\left(y^{\prime}+a^{\prime}\right)$ et

$$
\left|\alpha\left(1-\frac{a^{\prime}}{D_{1}} \frac{1}{\left(1+\alpha^{2}\right)^{1 / 2}}\right)+\operatorname{Re} \xi\right| \geq \frac{10}{\left|\cos \gamma_{+}\right|}-\delta_{1}
$$

donc $|\alpha|>5 / \cos \gamma_{+}$et on conclut comme précédemment.

On a donc à nouveau la décomposition (B.18) pour $\xi \in \mathscr{C}_{1}^{+}$. En intégrant par partie en $x^{\prime}$, on obtient donc, les contributions de $\partial \Sigma_{0}$ rentrant dans $J_{n}^{1}$,

$$
J_{n}=J_{n}^{0}+J_{n}^{1}
$$

où $J_{n}^{1}$ vérifie les estimées (B.9) et

$$
\begin{aligned}
J_{n}^{0}=\frac{\lambda}{2 \pi} \iint_{\Sigma_{0}} e^{i \lambda \alpha x^{\prime}-\lambda x^{\prime 2} / 2} \frac{d_{x}^{n} \sigma\left(0, x^{\prime}, \tau, \xi\right)}{n !} \\
\quad \times F_{+}\left(t-i \tau,-i \xi+x^{\prime}-i \alpha\right) d x^{\prime} d \alpha .
\end{aligned}
$$

Comme on a $a^{\prime} \ll d$, on a pour $\left(x^{\prime}, \alpha\right) \in \Sigma_{0}, \xi \in \mathscr{C}_{1}$,

$$
\mid F_{+}\left(t-i \tau,-i \xi+x^{\prime}-i \alpha \mid \leq D \lambda^{D} e^{\lambda \varphi_{0}(t-i \tau)}\right.
$$

et

$$
\left\|\partial_{z}^{m} F_{+}\left(t-i \tau,-i \xi+x^{\prime}-i \alpha\right)\right\|_{L^{2}\left(\mathscr{G}_{1}\right)} \leq \lambda^{D} e^{\lambda \varphi_{0}(t-i \tau)} D^{m+1} m !
$$

En écrivant le développement de Taylor sur $x^{\prime}=0$

$$
d_{x}^{n} \sigma\left(0, x^{\prime}\right)=\sum_{m \leq \lambda / C_{2}} \frac{d_{x}^{n} d_{x^{\prime}}^{m}}{m !} \sigma(0,0) x^{\prime m}+\frac{x^{M+1}}{M !} \int \cdots
$$

$M=E\left(\lambda / C_{2}\right)$ et en intégrant par parties en $\alpha$, on obtient une nouvelle décomposition

$$
J_{n}=\sum_{m \leq \lambda / C_{2}} J_{n, m}+J_{n}^{2}
$$




$$
\begin{aligned}
J_{n, m}=\frac{d_{x}^{n} d_{x^{\prime}}^{m} \sigma(0,0, \tau, \xi)}{n ! m !} \frac{\lambda}{2 \pi} \iint_{\Sigma_{0}} e^{i \lambda \alpha x^{\prime}-\lambda x^{\prime 2} / 2}\left(\frac{1}{\lambda} \partial_{z}\right)^{m} \\
\quad \times F_{+}\left(t-i \tau,-i \xi+x^{\prime}-i \alpha\right) d x d \alpha
\end{aligned}
$$

où $J_{n}^{2}$ vérifie toujours (B.9), le terme de reste avec $M=E\left(\lambda / C_{2}\right)$ étant estimé en norme $L^{2}\left(\mathscr{C}_{1}\right)$ par

$$
D \lambda^{D} e^{\lambda \varphi_{0}(t-i \tau)}\left(\frac{D_{1}}{a^{\prime}}\right)^{n+1}\left(\frac{D_{1}}{a^{\prime}}\right)^{M+1} \lambda^{-M / 2}\left(\frac{M}{2}\right) !
$$

et les termes provenant des intégrations par parties en $\alpha$ par

$$
D \lambda^{D} e^{\lambda \varphi_{0}(t-i \tau)}\left(\frac{D_{1}}{a^{\prime}}\right)^{n+1} \sum_{m \leq \lambda / C_{2}}\left(\frac{D_{1}}{a^{\prime}}\right)^{m+1} \lambda^{-m} e^{-r^{2} a^{\prime} \lambda / D D_{2}} D^{m} m !,
$$

qui rentrent dans $J_{n}^{2}$ pourvu que $C_{2} \geq C_{2}^{0}$, où $C_{2}^{0}$ dépend de $a^{\prime}$.

A ce stade, on peut remplacer dans (B.26) $\Sigma_{0}$ par tout bon contour localisé près de $x^{\prime}=\alpha=0$ pour la phase $\alpha x^{\prime}+i x^{\prime 2} / 2$; pour $\rho$ assez petit, on a aussi

$$
\begin{aligned}
& \frac{\lambda}{2 \pi} \iint_{\Sigma_{0}} e^{i \lambda \alpha x^{\prime}-\lambda x^{\prime 2} / 2} F_{+}\left(t-i \tau,-i \xi+x^{\prime}-i \alpha\right) d x^{\prime} d \alpha-\left(J_{\rho} F+\right)(t-i \tau,-i \xi) \\
& \quad=H(t-i \tau,-i \xi, \lambda),
\end{aligned}
$$

avec

$$
\left\|\partial_{z}^{m} H(t-i \tau, \cdot, \lambda)\right\|_{L^{2}\left(\mathscr{E}_{1}\right)} \leq C e^{-\lambda / C} D^{m} m ! e^{\lambda \varphi_{0}(t-i \tau)} .
$$

D’après le Lemme $4.3 \mathrm{du} \S 4.2$, on a

$$
\lambda \int_{0}^{\infty} e^{\lambda x(z+i \xi)} x^{n} \psi(x) e^{-\lambda x^{2} / 2} d x=\left(\frac{1}{\lambda} \partial_{z}\right)^{n}\left[J_{\rho}^{-1}\left(\frac{1}{z+i \xi}\right)+r(z+i \xi, \lambda)\right],
$$

avec pour $z \in U, \xi \in \mathscr{C}_{1},\left|\partial_{z}^{j} r\right| \leq C e^{-\lambda / C} j ! /|z|$. On obtient donc, en faisant tendre $\nu$ vers zéro, en remarquant $\left[J_{\rho}^{-1}, \partial_{z}\right]=\left[J_{\rho}, \partial_{z}\right]=0$,

$$
\begin{aligned}
I-J_{\rho}^{-1}\left(\sum_{\substack{m \leq \lambda / C_{2} \\
n \leq \lambda / C_{1}^{0}}} \frac{1}{2 \pi} \int_{\mathscr{C}_{1}}\left(\frac{1}{\lambda} \partial_{z}\right)^{n}\left(\frac{1}{z+i \xi}\right) \frac{d_{x}^{n} d_{x^{\prime}}^{m}}{n ! m !}\right. \\
\left.\times \sigma(0,0, \tau, \xi)\left(\frac{1}{\lambda} \partial_{z}\right)^{m}\left(J_{\rho} F_{+}\right)(-i \xi)\right)
\end{aligned}
$$

est négligeable dans $H\left(\varphi_{0}, X_{\kappa}, U\right)$, et cela reste valable en remplaçant $C_{1}^{0}$ par $C_{1} \geq C_{1}^{0}=e D_{1} / a^{\prime} ;$ d'où la Proposition.

Preuve de la Proposition 4.2, §4.4.

Pour $\operatorname{Im} \tau<0$, on pose

$$
I(t, \tau, z, \lambda)=T_{1}\left(S_{\lambda \tau}^{+,-} T_{0} f^{-}\right)(t-i \tau, z, \lambda)=\lim _{\nu \rightarrow 0^{+}} I^{\nu}(t, \tau, z, \lambda),
$$




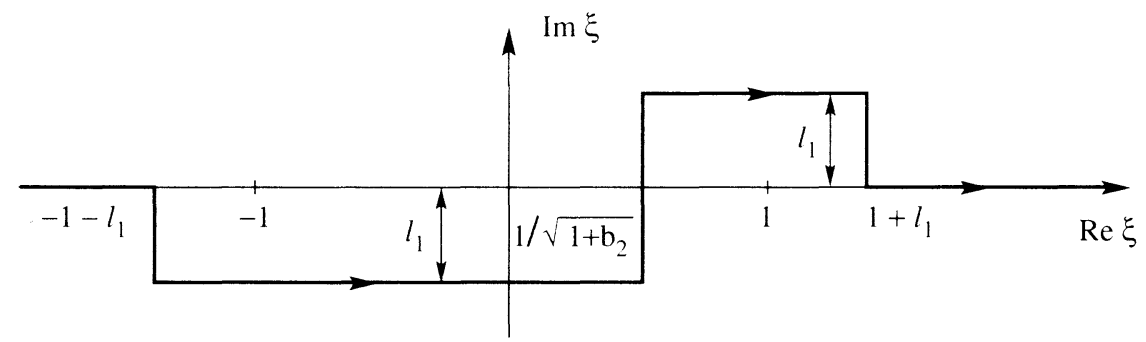

Figure 14

avec, compte tenu des expressions (2.38) et (2.41),

$$
I^{\nu}(t, \tau, z, \lambda)=\int_{-\infty}^{+\infty} d \xi e^{-\nu \xi^{2}} k(\tau, z, \xi, \lambda) j(t, \tau, \xi, \lambda),
$$

(B.30) $k(\tau, z, \xi, \lambda)=\int_{0}^{\infty} d x \psi(x)\left(1-a^{\prime}(x) \frac{\xi}{\xi_{0}}\right) e^{\lambda\left[x(z+i \xi)+i a(x) \xi_{0}-x^{2} / 2\right]}$,

$$
j(t, \tau, \xi, \lambda)=\frac{\lambda}{2 \pi} \int_{0}^{\infty} d x^{\prime} \psi\left(x^{\prime}\right) e^{-i \lambda x^{\prime} \xi-i \lambda b\left(x^{\prime}\right) \xi_{0}} T_{0} f_{-}\left(t-i \tau, x^{\prime}, \lambda\right) .
$$

Pour $l_{1}>0$, on introduit le contour $\mathscr{E}\left(l_{1}\right)$ représenté dans la Figure 14.

On suppose

$$
\kappa \ll l_{1} \ll 1 .
$$

Alors, pour $\xi \in \mathscr{C}\left(l_{1}\right)$, la fonction $\tau \mapsto \xi_{0}$ se prolonge en une fonction holomorphe sur $|\tau-1|<3 \kappa$, et il existe des constantes $D_{j}, j=2,3,4,5,6$, indépendantes de $l_{1}$ et $\kappa$, telles que

$$
\begin{gathered}
\operatorname{Im} \xi_{0} \geq-D_{2} l_{1}, \\
D_{3}\left(1+\operatorname{Im} \xi_{0}\right) \geq\left|\xi_{0}\right| \geq \frac{l_{1}^{2}+\kappa}{D_{3}}, \\
\operatorname{Im}\left(\xi+b \xi_{0}\right) \leq D_{4} \kappa, \\
\operatorname{Im}\left(\xi+b \xi_{0}\right) \geq-\frac{l_{1}}{D_{5}} \Rightarrow\left|\xi-\frac{1}{\sqrt{1+b^{2}}}\right| \leq \sqrt{l_{1}}, \quad \text { et } \\
\left|\xi+b \xi_{0}-\sqrt{1+b^{2}}\right| \leq D_{6} l_{1} .
\end{gathered}
$$

Les estimations (B.35) et (B.36) s'obtiennent aisément en tenant compte du contour décrit par $\zeta=\xi+b \xi_{0}$, représenté dans la Figure 15, p. 406, pour $\tau=1$. En déformant l'intégrale (B.29) sur $\mathscr{C}\left(l_{1}\right)$, on constate que $I^{\nu}$ est définie pour $(t, \tau) \in X_{\kappa}, z \in U$. D'autre part, en posant

$$
a(x)=x p(x), \quad b\left(x^{\prime}\right)=x^{\prime} q\left(x^{\prime}\right)
$$

on constate que, si $t_{0}$ est assez petit, 


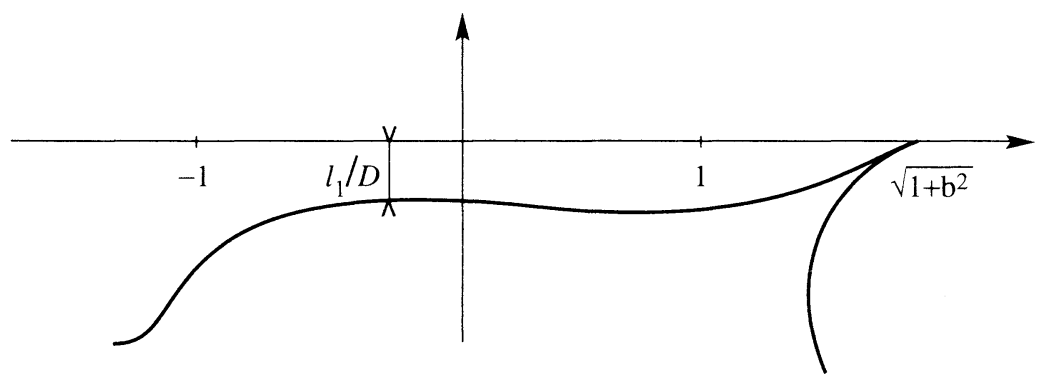

FIGURE 15

$$
\frac{a}{2} \leq p(x) \leq \frac{3 a}{2}, \quad \frac{3 b}{2} \leq q\left(x^{\prime}\right) \leq \frac{b}{2},
$$

pour $x \in \operatorname{supp}(\psi), x^{\prime} \in \operatorname{supp}(\psi)$. On en déduit que, pour $\xi \in \mathscr{C}\left(l_{1}\right), z \in U$, on a, compte tenu de (B.33),

$$
\operatorname{Re}\left(z+i\left(\xi+p(x) \xi_{0}\right)\right) \leq-4+D l_{1} \leq-1,
$$

$$
\operatorname{Re}\left(-i\left(\xi+q\left(x^{\prime}\right) \xi_{0}\right) \leq D l_{1}\right.
$$

De (B.40), on déduit alors que

$$
|j(t, \tau, \xi, \lambda)| \leq D \lambda^{D} e^{\lambda D l_{1} t_{0}}\left\|f_{-}\right\|_{L^{2}} e^{\lambda \varphi_{0}(t-i \tau)},
$$

tandis que l'on écrit (B.30) sous la forme

$$
k(\tau, z, \xi, \lambda)=\sum_{n=0}^{N} k_{n}(\tau, z, \xi, \lambda)+k_{n, R}(\tau, z, \xi, \lambda),
$$

avec, en posant $a(x)=a x+r_{+}(x)$,

$$
k_{n}(\tau, z, \xi, \lambda)=\int_{0}^{\infty} d x \psi(x) e^{\lambda x\left(z+i \xi+i a \xi_{0}\right)} \frac{x^{n}}{n !} \sigma_{n}(0, \xi, \tau, \lambda),
$$

$$
\begin{aligned}
& k_{N, R}(\tau, z, \xi, \lambda) \\
& \quad=\int_{0}^{\infty} d x \psi(x) e^{\lambda x\left(z+i \xi+i a \xi_{0}\right)} \int_{0}^{x} \frac{(x-y)^{N}}{N !} \sigma_{N+1}(y, \xi, \tau, \lambda) d y, \\
& \quad \sigma_{n}(y, \xi, \tau, \lambda)=\partial_{y}^{n}\left(e^{\lambda\left(i r_{+}(y) \xi_{0}-y^{2} / 2\right)}\left(1-a^{\prime}(y) \frac{\xi}{\xi_{0}}\right)\right) .
\end{aligned}
$$

Soit $\rho>0$ assez petit pour que $a$ soit holomorphe sur $\{z, d(z, \operatorname{supp}(\psi)) \leq$ $\rho\}$. Alors, pour $y \in \operatorname{supp}(\psi)$, on a

$$
\begin{aligned}
& \operatorname{Re}\left[i r_{+}\left(y+\rho e^{i \theta}\right) \xi_{0}-\frac{\left(y+\rho e^{i \theta}\right)^{2}}{2}\right] \\
& \quad \leq \operatorname{Re}\left(i r_{+}(y) \xi_{0}\right)-\frac{y^{2}}{2}+D\left(y \rho+\rho^{2}\right)\left(1+\left|\xi_{0}\right|\right),
\end{aligned}
$$


d'où, en posant $\zeta=z+i \xi+i a \xi_{0}$,

$$
\begin{aligned}
\left|k_{N, R}\right| \leq & \int_{0}^{\infty} \int_{0}^{x} d x d y \psi(x)(x-y)^{N} e^{\lambda(x-y) \operatorname{Re} \zeta+\lambda \phi(y)} \\
& \times(N+1) \rho^{-(N+1)} e^{\lambda D \rho^{2}\left(1+\left|\xi_{0}\right|\right)}
\end{aligned}
$$

avec $\phi(y)=\operatorname{Re}\left(y\left(z+i \xi+i p(y) \xi_{0}\right)\right)+D y \rho\left(1+\left|\xi_{0}\right|\right)$ donc, d'après (B.34),

$$
\phi(y) \leq y \operatorname{Re}\left(z+D_{3} \rho+i \xi+i\left(p(y)+D_{3} \rho\right) \xi_{0}\right) \leq 0
$$

si $\rho \ll 1$, compte tenu de (B.33) et de (B.38).

Posant $x-y=u$ dans (B.47), il vient:

$$
\begin{aligned}
\left|k_{N, R}(\tau, z, \xi, \lambda)\right| \leq & D\left(\int_{0}^{\infty} d u u^{N} e^{\lambda u \operatorname{Re} \zeta}\right)(N+1) \\
& \times \rho^{-(N+1)} e^{\lambda D \rho^{2}\left(1+\left|\xi_{0}\right|\right)} \\
\leq & D \frac{(N+1) !}{(-\lambda \operatorname{Re} \zeta)^{N+1}} \rho^{-(N+1)} e^{\lambda D \rho^{2}\left(1+\left|\xi_{0}\right|\right)}
\end{aligned}
$$

On choisit alors $N+1=\left[\lambda / C_{0}\right]$, avec $C_{0} \gg 1$, et $\rho=\left((N+1) / \lambda\left(1+\left|\xi_{0}\right|\right)\right)^{1 / 2}$. Il vient

$$
\left|k_{N, R}(\tau, z, \xi, \lambda)\right| \leq\left(\frac{(N+1) D}{\lambda}\right)^{(N+1) / 2} \frac{\left(1+\left|\xi_{0}\right|\right)^{(N+1) / 2}}{\left(|\operatorname{Re} z|+\operatorname{Im} \xi_{0}\right)^{(N+1)}}
$$

Fixant $C_{0} \geq e D$, on obtient, compte tenu de (B.34)

$$
\left|k_{N, R}(\tau, z, \xi, \lambda)\right| \leq D e^{-\lambda / 2 C_{0}}\left(|\operatorname{Re} z|+\operatorname{Im} \xi_{0}\right)^{-(N+1) / 2} .
$$

En comparant avec (B.41), on obtient que la contribution de $k_{N, R}$ dans $I^{\nu}$ est uniformément négligeable dans $H\left(\varphi_{0}, X_{\kappa}, U\right)$, pourvu que

$$
l_{1} t_{0} \ll \frac{1}{C_{0}}
$$

On estime de la même manière $k_{n}(\tau, z, \xi$, $\lambda)$, i.e.,

$$
\frac{1}{n !}\left|\sigma_{n}(0, \xi, \tau, \lambda)\right| \leq D \rho^{-n} e^{D \lambda \rho^{2}\left(1+\left|\xi_{0}\right|\right)}
$$

d'où avec $\rho=\left(n / \lambda\left(1+\left|\xi_{0}\right|\right)\right)^{1 / 2}$,

$$
\left|k_{n}(\tau, z, \xi, \lambda)\right| \leq D e^{-n / 2}\left(|\operatorname{Re} z|+\operatorname{Im} \xi_{0}\right)^{-(1+n / 2)},
$$

estimation qui nous sera utile plus loin.

Passons maintenant au développement de $j$. On décompose $\mathscr{B}\left(l_{1}\right)$ en deux parties $\mathscr{C}\left(l_{1}\right)^{+}$et $\mathscr{C}\left(l_{1}\right)^{-}$, où $\mathscr{E}\left(l_{1}\right)^{-}$est caractérisée par

$$
\operatorname{Im}\left(\xi+b \xi_{0}\right) \leq-\frac{l_{1}}{D_{5}} .
$$


En notant $r_{-}\left(x^{\prime}\right)=b\left(x^{\prime}\right)-b x^{\prime}$, et en utilisant la formule d'inversion (4.5), on a

$$
\begin{aligned}
j(t, \tau, \xi, \lambda)= & \left(\frac{\lambda}{2 \pi}\right)^{2} \int_{-\infty}^{+\infty} \int_{0}^{+\infty} e^{i \lambda x^{\prime} \alpha-i \lambda r_{-}\left(x^{\prime}\right) \xi_{0}-\lambda x^{\prime 2} / 2} \psi\left(x^{\prime}\right) \\
& \times F_{-}\left(t-i \tau, x^{\prime}-i\left(\xi+b \xi_{0}\right)-i \alpha, \lambda\right) d x^{\prime} d \alpha .
\end{aligned}
$$

Supposons d'abord $\xi \in \mathscr{C}\left(l_{1}\right)^{-}$, et déformons l'intégrale en $\left(\alpha, x^{\prime}\right)$ sur le contour $\Gamma\left(l_{2}\right): \alpha=\beta+i l_{2}\left(1+\left|\operatorname{Im} \xi_{0}\right|\right)$, avec $l_{2}>0$ à choisir. Alors, pour $\alpha \in \Gamma\left(l_{2}\right)$,

(B.56) $\operatorname{Re}\left(x^{\prime}-i\left(\xi+b \xi_{0}\right)-i \alpha\right) \leq \begin{cases}4 c t_{0}-\frac{l_{1}}{D_{5}}+3 l_{2} & \text { si }\left|\operatorname{Im} \xi_{0}\right| \leq 2, \\ 4 c t_{0}+l_{2}+2\left(b-l_{2}\right) & \text { si }\left|\operatorname{Im} \xi_{0}\right| \geq 2,\end{cases}$

car alors $\xi \in \mathbb{R},|\xi| \geq 1+l_{1}$ et $\operatorname{Im} \xi_{0}>0$.

En imposant

$$
t_{0}+l_{2} \ll l_{1} \ll 1
$$

on déduit que

$$
\operatorname{Re}\left(x^{\prime}-i\left(\xi+b \xi_{0}\right)-i \alpha\right) \leq-\frac{l_{1}}{D}
$$

donc, d'après (4.12),

(B.59) $\left\|\partial_{z}^{m} F_{-}\left(t-i \tau, x^{\prime}-i\left(\xi+b \xi_{0}\right)-i \alpha, \lambda\right)\right\|_{L^{2}\left(\Gamma\left(l_{2}\right)\right)} \leq\left(\frac{D}{l_{1}}\right)^{m+1} m ! e^{\lambda \varphi_{0}(t-i \tau)}$.

Par ailleurs

$$
\begin{aligned}
\operatorname{Re}\left(i x^{\prime} \alpha-i r_{-}\left(x^{\prime}\right) \xi_{0}-\frac{x^{\prime 2}}{2}\right) & \leq-x^{\prime} l_{2}\left(1+\left|\operatorname{Im} \xi_{0}\right|\right)+D x^{\prime 2}\left|\operatorname{Im} \xi_{0}\right| \\
& \leq-x^{\prime} \frac{l_{2}}{2}
\end{aligned}
$$

si

(B.61)

$$
t_{0} \ll l_{2}
$$

On écrit alors, pour $\alpha \in \Gamma\left(l_{2}\right)$,

$$
\begin{aligned}
\int_{0}^{\infty} e^{i \lambda x^{\prime} \alpha-\lambda x^{\prime 2} / 2-i \lambda r_{-}\left(x^{\prime}\right) \xi_{0}} \psi\left(x^{\prime}\right) F_{-} d x^{\prime} & +R_{M}(t, \tau, \xi, \alpha, \lambda), \\
= & \left.\sum_{m=0}^{M}\left(\int_{0}^{\infty} e^{i \lambda x^{\prime} \alpha} \psi\left(x^{\prime}\right) \frac{x^{\prime m}}{m !} d x^{\prime}\right) \partial_{x^{\prime}}^{m}\left(e^{-\lambda x^{\prime 2} / 2-i \lambda r_{-} \xi_{0}} F_{-}\right)\right|_{x^{\prime}=0} \\
R_{M}= & \int_{0}^{\infty} d x^{\prime} \psi\left(x^{\prime}\right) e^{i \lambda x^{\prime} \alpha} \\
& \times \int_{0}^{x^{\prime}} \frac{\left(x^{\prime}-y^{\prime}\right)^{M}}{M !} \partial_{y^{\prime}}^{M+1}\left(e^{-\lambda y^{\prime 2} / 2-i \lambda r_{-}\left(y^{\prime}\right) \xi_{0}} F_{-}\left(y^{\prime}\right)\right) d y^{\prime} .
\end{aligned}
$$


Pour obtenir une estimation de $R_{M}$ qui soit $L^{1}$ en $\alpha$, on intègre par parties en $x^{\prime}$ :

$$
\begin{aligned}
R_{M}= & -\frac{1}{i \lambda \alpha} \int_{0}^{\infty} d x^{\prime} \psi\left(x^{\prime}\right) e^{i \lambda x^{\prime} \alpha} \\
& \times \int_{0}^{x^{\prime}} \frac{\left(x^{\prime}-y^{\prime}\right)^{M-1}}{(M-1) !} \partial_{y^{\prime}}^{M+1}\left(e^{-\lambda y^{\prime 2} / 2-i \lambda r_{-}\left(y^{\prime}\right) \xi_{0}} F_{-}\left(y^{\prime}\right)\right) d y^{\prime} .
\end{aligned}
$$

Si $\rho \ll \inf \left(l_{2}, l_{1}\right)=l_{2}$, on a, comme dans (B.48),

$$
\begin{aligned}
\int_{\Gamma\left(l_{2}\right)} & \left|R_{M}(t, \tau, \xi, \alpha, \lambda)\right| d \alpha \\
& \leq D e^{\lambda \varphi_{0}(t-i \tau)} \frac{(M+1) ! \rho^{-(M+1)}}{\lambda^{M+1}\left(l_{2}\left(1+\left|\operatorname{Im} \xi_{0}\right|\right)\right)^{M+1 / 2}} e^{\lambda D \rho^{2}\left(1+\left|\xi_{0}\right|\right)}
\end{aligned}
$$

compte tenu de (B.59) et du fait que

$$
\left(\int_{\Gamma\left(l_{2}\right)} \frac{d \alpha}{|\alpha|^{2}}\right)^{1 / 2} \leq \frac{D}{\left(l_{2}\left(1+\left|\operatorname{Im} \xi_{0}\right|\right)^{1 / 2}\right.} .
$$

On choisit alors $M+1=\left[\lambda / C_{0}^{\prime}\right]$ avec $C_{0}^{\prime} \gg 1 / l_{2}^{2}$, et $\rho=\left((M+1) / \lambda\left(1+\left|\xi_{0}\right|\right)\right)^{1 / 2}$. Alors

$$
\int_{\Gamma\left(l_{2}\right)}\left|R_{M}\right| d \alpha \leq D e^{-\lambda / 2 C_{0}^{\prime}}\left(1+\left|\operatorname{Im} \xi_{0}\right|\right)^{-M / 2},
$$

ce qui, compte tenu de (B.53) entraîne que la contribution de $R_{M}$ dans $I$ est négligeable. Revenons à (B.62). On a

$$
\begin{gathered}
\int_{0}^{\infty} e^{i \lambda x^{\prime} \alpha} \psi\left(x^{\prime}\right) \frac{x^{\prime m}}{m !} d x^{\prime}=\left(-\frac{1}{i \lambda \alpha}\right)^{m+1}+r_{m}(\alpha) \\
\left|r_{m}(\alpha)\right|=\left|\frac{1}{i \lambda \alpha} \int_{0}^{\infty} e^{i \lambda x^{\prime} \alpha} \partial_{x^{\prime}}\left((1-\psi) \frac{x^{\prime m}}{m !}\right) d x^{\prime}\right| \\
\leq \frac{1}{\lambda|\alpha|} e^{-2 l_{2} c t_{0}\left(1+\left|\operatorname{Im} \xi_{0}\right|\right) \lambda}\left(\frac{D}{\lambda l_{2}\left(1+\left|\operatorname{Im} \xi_{0}\right|\right)}\right)^{m-1}
\end{gathered}
$$

Comme par ailleurs, d'après (B.59),

$$
\begin{aligned}
& \left.\left|\partial_{x^{\prime}}^{m}\left(e^{-\lambda x^{\prime 2} / 2-i \lambda r_{-}\left(x^{\prime}\right) \xi_{0}} F_{-}\left(x^{\prime}, \alpha\right)\right)\right|_{x^{\prime}=0}\right|_{L^{2}\left(\Gamma\left(l_{2}\right)\right)} \\
& \quad \leq m ! \rho^{-m} e^{\lambda \varphi_{0}(t-i \tau)} \times e^{\lambda D \rho^{2}\left(1+\left|\xi_{0}\right|\right)}, \quad \text { avec } \rho \ll l_{1},
\end{aligned}
$$

on a, en choisissant

$$
\rho=\left(\frac{m}{\lambda\left(1+\left|\xi_{0}\right|\right)}\right)^{1 / 2} \ll l_{2},
$$


et puisque $C_{0}^{\prime} \ll l_{2}^{-2}$,

$$
\begin{gathered}
\sum_{m \leq \lambda / C_{0}^{\prime}} \int\left|r_{m}(\alpha)\right|\left|\partial_{x^{\prime}}^{m}\left(e^{-\lambda x^{\prime 2} / 2-i \lambda r_{-} \xi_{0}} F^{-}\right)\right|_{x^{\prime}=0} \mid d \alpha \\
\leq D e^{-\lambda\left(1+\left|\xi_{0}\right|\right) / D} e^{\lambda \varphi_{0}(t-i \tau)},
\end{gathered}
$$

ce qui donne une contribution négligeable dans $I$ compte tenu de (B.53). En conclusion, si $\xi \in \mathscr{C}\left(l_{2}\right)^{-}$, on a

$$
\begin{aligned}
j(t, \tau, \xi, \lambda) \sim \sum_{m \leq \lambda / C_{0}^{\prime}}\left(\frac{\lambda}{2 \pi}\right)^{2} \int_{\Gamma\left(l_{2}\right)} & \left(-\frac{1}{i \lambda \alpha}\right)^{m+1} \\
& \times\left.\partial_{x^{\prime}}^{m}\left(e^{-\lambda x^{\prime 2} / 2-i \lambda r_{-} \xi_{0}} F_{-}\right)\right|_{x^{\prime}=0} d \alpha
\end{aligned}
$$

soit, par la formule de Cauchy,

$$
\begin{array}{r}
\left.j(t, \tau, \xi, \lambda) \sim \frac{\lambda}{2 \pi} \sum_{m \leq \lambda / C_{0}^{\prime}} \lambda^{-m} \frac{1}{m !} \partial_{x^{\prime}}^{m}\left(e^{-\lambda x^{\prime 2} / 2-i \lambda r_{-}\left(x^{\prime}\right) \xi_{0}} \partial_{z}^{m} F_{-}\right)\right|_{x^{\prime}=0} \\
\left(t-i \tau,-i\left(\xi+b \xi_{0}\right), \lambda\right) .
\end{array}
$$

Supposons maintenant $\xi \in \mathscr{C}\left(l_{1}\right)^{+}$, et revenons à la formule (B.55). Puisque $T_{0} f_{-}\left(t-i \tau, x^{\prime}, \lambda\right)=0$ si $x^{\prime}<0$, on peut également écrire

$$
\begin{aligned}
j(t, \tau, \xi, \lambda)= & \left(\frac{\lambda}{2 \pi}\right)^{2} \iint_{\mathbb{R}^{2}} e^{i \lambda x^{\prime} \alpha-i \lambda r_{-}\left(x^{\prime}\right) \xi_{0}-\lambda x^{\prime 2} / 2} \psi\left(x^{\prime}\right) \\
& \quad \times F_{-}\left(t-i \tau, x^{\prime}-i\left(\xi+b \xi_{0}\right)-i \alpha, \lambda\right) d x^{\prime} d \alpha \\
= & \frac{\lambda}{2 \pi} \iint_{\mathbb{R}^{2}} \sigma\left(t, \tau, \xi, \lambda ; x^{\prime}, \alpha\right) d x^{\prime} d \alpha,
\end{aligned}
$$

avec, après deux intégrations par parties en $x^{\prime}$

$$
\begin{aligned}
\sigma= & \frac{\lambda}{2 \pi} \frac{e^{i \lambda x^{\prime} \alpha}}{1+\alpha^{2}}\left(1-\frac{1}{\lambda^{2}} \partial_{x^{\prime}}^{2}\right) \\
& \times\left(e^{-\lambda x^{\prime 2} / 2-i \lambda r_{-}\left(x^{\prime}\right) \xi_{0}} \psi\left(x^{\prime}\right) F_{-}\left(t-i \tau, x^{\prime}-i\left(\xi+b \xi_{0}\right)-i \alpha, \lambda\right)\right) .
\end{aligned}
$$

On procède alors comme dans la démonstration de la Proposition $4.1 \mathrm{du} \S 4.3$. On introduit $g \in C_{0}^{\infty}(]-2 c t_{0}, 2 c t_{0}[), 0 \leq g \leq 1, g \equiv 1$ sur $\left[-c t_{0}, c t_{0}\right]$, et le contour

$$
\Sigma=\left\{\left(\alpha, x^{\prime}\right) ; \alpha \in \mathbb{R}, x^{\prime}=y^{\prime}+i g\left(y^{\prime}\right) \frac{\alpha}{\sqrt{1+\alpha^{2}}} l_{3}, y^{\prime} \in \mathbb{R}\right\}
$$

avec $0<l_{3} \ll 1$. D'après l'estimation (4.14), on a, pour $\xi \in \mathscr{C}\left(l_{1}\right)^{+}$,

$$
\left|\sigma\left(t, \tau, \xi, \lambda ; x^{\prime}, \alpha\right)\right| \leq D \lambda^{D} \frac{\left\|f_{-}\right\|_{L^{2}}}{1+\alpha^{2}} e^{\lambda \varphi_{0}(t-i \tau)} e^{\lambda G},
$$




$$
\begin{aligned}
G= & -g\left(y^{\prime}\right) \frac{\alpha^{2}}{\sqrt{1+\alpha^{2}}} l_{3}-\frac{y^{\prime 2}}{2}+g^{2} l_{3}^{2} \frac{\alpha^{2}}{1+\alpha^{2}} \\
& +\operatorname{Im}\left(r_{-}\left(x^{\prime}\right) \xi_{0}\right)+\Phi_{\delta}^{+}(z),
\end{aligned}
$$

(B.80)

$$
z=y^{\prime}+\operatorname{Im}\left(\xi+b \xi_{0}\right)-i\left(\operatorname{Re}\left(\xi+b \xi_{0}\right)+\alpha\left(1-g\left(y^{\prime}\right) \frac{l_{3}}{\sqrt{1+\alpha^{2}}}\right)\right) .
$$

Compte tenu de (B.36), on a

$$
\begin{aligned}
\operatorname{Im}\left(r_{-}\left(x^{\prime}\right) \xi_{0}\right)= & r_{-}\left(y^{\prime}\right) \operatorname{Im} \xi_{0}+r_{-}^{\prime}\left(y^{\prime}\right) \operatorname{Im}\left(i l_{3} g\left(y^{\prime}\right) \frac{\alpha}{\sqrt{1+\alpha^{2}}} \xi_{0}\right) \\
& +O\left(l_{3}^{2} g\left(y^{\prime}\right)^{2} \frac{\alpha^{2}}{1+\alpha^{2}}\right) \\
\leq & D\left(y^{\prime 2} l_{1}^{1 / 2}+y^{\prime} l_{3} g\left(y^{\prime}\right) \frac{\alpha}{\sqrt{1+\alpha^{2}}}+l_{3}^{2} g\left(y^{\prime}\right)^{2} \frac{\alpha^{2}}{1+\alpha^{2}}\right) \\
\leq & D\left(y^{\prime 2}\left(l_{1}^{1 / 2}+l_{3}^{1 / 2}\right)+g\left(y^{\prime}\right)^{2} \frac{\alpha^{2}}{1+\alpha^{2}} l_{3}^{3 / 2}\right),
\end{aligned}
$$

de sorte que

$$
\begin{gathered}
G \leq-l_{3} g\left(y^{\prime}\right) \frac{\alpha^{2}}{\sqrt{1+\alpha^{2}}}\left(1-D l_{3}^{1 / 2}\right)-\frac{y^{\prime 2}}{2}\left(1-l_{4}\right)+\Phi_{\delta}^{+}(z), \\
l_{4} \leq D\left(l_{1}^{1 / 2}+l_{3}^{1 / 2}\right) .
\end{gathered}
$$

Nous allons vérifier que $G<0$, en suivant une discussion très analogue à celle de la preuve de la Proposition $4.1 \mathrm{du} \S 4.3$, et utilisant les estimations (B.35) et (B.36). Par (B.35), on a

$$
\operatorname{Re} z \leq y^{\prime}+D_{4} \kappa .
$$

Si $y^{\prime} \leq-c t_{0}$, alors $\operatorname{Re} z<0$ puisque

$$
\kappa \ll t_{0} .
$$

On en déduit $\Phi_{\delta}^{+}(z)=0$ et $G<0$ par (B.82).

Si $y^{\prime} \geq c t_{0}$, alors $y^{\prime}+D_{4} \kappa \geq \delta$ et

$$
\Phi_{\delta}^{+}(z) \leq \Phi_{\delta}\left(y^{\prime}+D_{4} \kappa\right)
$$

$$
=\frac{\left(y^{\prime}+D_{4} \kappa\right)^{2}}{2}-\frac{1}{2\left(1+c^{2}\right)}\left(y^{\prime}+D_{4} \kappa-\delta\right)^{2},
$$

$$
\begin{aligned}
G & \leq-\frac{y^{\prime 2}}{2}\left(1-l_{4}\right)+\frac{\left(y^{\prime}+D_{4} \kappa\right)^{2}}{2}-\frac{1}{2\left(1+c^{2}\right)}\left(y^{\prime}+D_{4} \kappa-\delta\right)^{2} \\
& =h\left(y^{\prime}\right) .
\end{aligned}
$$


Notons que

$$
\begin{aligned}
h^{\prime}\left(y^{\prime}\right) & =-y^{\prime}\left(1-l_{4}\right)+y^{\prime}+D_{4} \kappa-\frac{1}{1+c^{2}}\left(y^{\prime}+D_{4} \kappa-\delta\right) \\
& =y^{\prime}\left(l_{4}-\frac{1}{1+c^{2}}\right)+D \kappa \\
& <0
\end{aligned}
$$

si $y^{\prime} \geq c t_{0}$, d'après $l_{4} \ll 1$ et $(\mathrm{B} .85)$.

D'autre part

$$
h\left(y^{\prime}\right) \leq D\left(\kappa y^{\prime}+\kappa^{2}\right)-\frac{y^{\prime 2}}{2}\left(\frac{1}{1+c^{2}}-l_{4}\right)
$$

donc $h\left(c t_{0}\right)<0$ d'après $l_{4} \ll 1$ et (B.85). Il en résulte que $h\left(y^{\prime}\right)<0$ si $y^{\prime} \geq c t_{0}$, donc $G<0$.

Enfin, si $\left|y^{\prime}\right| \leq c t_{0}$, alors $g\left(y^{\prime}\right)=1$. Choisissons $\delta_{1}$ tel que

$$
\kappa \ll \delta_{1} \ll d .
$$

Compte tenu de (B.84), si $y^{\prime}+D_{4} \kappa \leq 0$ alors $\Phi_{\delta}^{+}=0$ et $G<0$ par (B.82). Si $y^{\prime}+D_{4} \kappa \geq \delta_{1}$ alors

$$
\Phi_{\delta}^{+}(z) \leq \Phi_{\delta}\left(y^{\prime}+D_{4} \kappa\right), \quad G \leq h\left(y^{\prime}\right)<0
$$

comme ci-dessus, car $\kappa \ll \delta_{1}$. Enfin, si $y^{\prime}+D_{4} \kappa \in\left[0, \delta_{1}\right]$, on a, en utilisant l'estimation (4.14),

—soit $\Phi_{\delta}^{+}(z)=0$, et $G<0$ par (B.82),

-soit $\left|\operatorname{Im} z-\sqrt{1+b^{2}}\right| \leq \varepsilon_{0}$ et, par (B.80), (B.36) et $l_{3} \ll 1, \alpha$ est de l'ordre de $-2 \sqrt{1+b^{2}}$; soit $|z| \geq 10 \sqrt{1+b^{2}}$, et $|\alpha| \geq 8 \sqrt{1+b^{2}}$; d'où, dans les deux cas

$$
\begin{aligned}
G\left(y^{\prime}\right) & \leq-\frac{l_{3}}{D}-\frac{y^{\prime 2}}{2}\left(1-l_{4}\right)+\frac{\left(y^{\prime}+D_{4} \kappa\right)^{2}}{2} \\
& \leq-\frac{l_{3}}{D}+D \kappa+\frac{y^{\prime 2}}{2} l_{4}<0
\end{aligned}
$$

si l'on impose

$$
\kappa \ll l_{3}, \quad \delta_{1}^{2} l_{4} \ll l_{3},
$$

ce qui, compte tenu de (B.83), est vérifié si

$$
\kappa \ll l_{3}, \quad \delta_{1} \ll l_{3}^{1 / 2} .
$$

En résumé, les conditions sur $\kappa, l_{1}, l_{2}, l_{3}, t_{0}, \delta_{1}$ sont, compte tenu de (B.32), (B.57), (B.61), (B.85), (B.90), et (B.94):

$$
\kappa \ll t_{0} \ll l_{2} \ll l_{1}, \quad \kappa \ll l_{3}, \quad \kappa \ll \delta_{1} \ll \operatorname{Inf}\left(l_{3}^{1 / 2}, d\right),
$$


ce qui est possible à réaliser. On conclut que, pour $\xi \in \mathscr{C}\left(l_{1}\right)^{+}$, on a, pour tout $r>0$, si $\Sigma(r)=\Sigma \cap\left\{\left|x^{\prime}\right|+|\alpha| \leq r\right\}$,

$$
j(t, \tau, \xi, \lambda)=\frac{\lambda}{2 \pi} \iint_{\Sigma(r)} \sigma d x^{\prime} d \alpha+O\left(e^{\lambda\left(\varphi_{0}-1 / C(r)\right)}\right),
$$

et la contribution du reste à $I$ est négligeable compte tenu de (B.53). On peut maintenant intégrer par parties en $x^{\prime}$ pour remplacer $\sigma$ par

$$
\begin{aligned}
\sigma_{0}\left(t, \tau, \xi, \lambda ; x^{\prime}, \alpha\right)= & \frac{\lambda}{2 \pi} e^{i \lambda\left(x^{\prime} \alpha+i x^{\prime 2} / 2-r_{-}\left(x^{\prime}\right) \xi_{0}\right)} \\
& \times F_{-}\left(t-i \tau, x^{\prime}-i \alpha-i\left(\xi+b \xi_{0}\right), \lambda\right) .
\end{aligned}
$$

Compte tenu de (B.35), $\kappa \ll d$ et de (4.14), on a

$$
\left|\partial_{z}^{m} F_{-}\left(t-i \tau, x^{\prime}-i \alpha-i\left(\xi+b \xi_{0}\right), \lambda\right)\right| \leq D^{m+1} m ! e^{\lambda \varphi_{0}(t-i \tau)},
$$

et on peut donc développer $\iint_{\Sigma(r)} \sigma_{0} d x^{\prime} d \alpha$ par la méthode de la phase stationnaire [S2]:

(B.99)

$$
\begin{aligned}
j(t, \tau, \xi, \lambda)= & \left.\frac{\lambda}{2 \pi} \sum_{m \leq \lambda / C_{0}^{\prime \prime}} \frac{1}{m ! \lambda^{m}}\left(\partial_{x^{\prime}} \partial_{z}+A \partial_{z}^{2}\right)^{m} v\left(t, \tau, \xi ; x^{\prime}, z, \lambda\right)\right|_{x^{\prime}=z=0} \\
& +O\left(e^{\lambda \varphi_{0}-\lambda / D}\right)
\end{aligned}
$$

$$
\begin{gathered}
A=-\frac{1}{2}\left(1+i a^{\prime \prime}(0) \xi_{0}\right), \\
v\left(t, \tau, \xi ; x^{\prime}, z, \lambda\right)=e^{-i \lambda \xi_{0}\left(a\left(x^{\prime}\right)-a x^{\prime}-a^{\prime \prime}(0) x^{\prime 2} / 2\right)} \\
\quad \times F_{-}\left(t-i \tau,-i\left(\xi+b \xi_{0}\right)+x^{\prime}+z, \lambda\right) .
\end{gathered}
$$

La constante $C_{0}^{\prime \prime}$ est ici supposée assez grande. Notons que, si $\rho \ll 1$,

$$
\left|\partial_{x^{\prime}}^{j} \partial_{z}^{k} v\right| \leq D e^{D \lambda \rho^{3}} \rho^{-j-k}(j+k) ! e^{\lambda \varphi_{0}(t-i \tau)},
$$

ce qui, en choisissant $\rho=(m / \lambda)^{1 / 3}$, montre que l'on peut remplacer dans (B.99) $C_{0}^{\prime \prime}$ par $C>C_{0}^{\prime \prime}$.

Il reste à comparer le développement (B.99) obtenu pour $\xi \in \mathscr{C}\left(l_{1}\right)^{+}$avec (B.74), obtenu pour $\xi \in \mathscr{C}\left(l_{1}\right)^{-}$, et que l'on peut réécrire, compte tenu de (B.100), (B.101),

$$
\left.j(t, \tau, \xi, \lambda) \sim \frac{\lambda}{2 \pi} \sum_{m \leq \lambda / C_{0}^{\prime}} \frac{1}{m ! \lambda^{m}}\left(\partial_{x^{\prime}} \partial_{z}\right)^{m}\left(e^{\lambda A x^{\prime 2}} v\left(t, \tau, \xi ; x^{\prime}, z, \lambda\right)\right)\right|_{x^{\prime}=z=0} .
$$

On va voir que (B.103) est également valable pour $\xi \in \mathscr{C}\left(l_{1}\right)^{+}$, quitte à remplacer $C_{0}^{\prime}$ par un plus grand $C$ (ce qui ne change rien à la validité de (B.103) pour $\left.\xi \in \mathscr{C}\left(l_{1}\right)^{-}\right)$. 
Soit donc $\xi \in \mathscr{C}\left(l_{1}\right)^{+}$, et soit $C>\max \left(C_{0}^{\prime}, C_{0}^{\prime \prime}\right)$. On pose, pour $X \in \mathbb{C}$,

(B.104)

$$
\begin{gathered}
P_{C}(X)=\left.\sum_{m \leq \lambda / C} \frac{1}{m ! \lambda^{m}}\left(\partial_{x^{\prime}} \partial_{z}+X \partial_{z}^{2}\right)^{m} v\right|_{x^{\prime}=z=0}, \\
Q_{C}(X)=\left.\sum_{m \leq \lambda / C} \frac{1}{m ! \lambda^{m}}\left(\partial_{x^{\prime}} \partial_{z}\right)^{m}\left(e^{\lambda X x^{\prime 2}} v\right)\right|_{x^{\prime}=z=0} .
\end{gathered}
$$

Alors $P_{C}$ et $Q_{C}$ sont des polynômes de degrés $\leq \lambda / C$ et $\leq \lambda / 2 C$, et

(B.105) si $k \leq \lambda / C$,

$$
\left(\frac{d}{d X}\right)^{k} P_{C}(0)=\left.\sum_{k \leq m \leq \lambda / C} \frac{1}{(m-k) ! \lambda^{m}} \partial_{x^{\prime}}^{m-k} \partial_{z}^{m+k} v\right|_{x^{\prime}=z=0}
$$

(B.106) si $k \leq \lambda / 2 C$,

$$
\begin{aligned}
\left(\frac{d}{d X}\right)^{k} Q_{C}(0) & =\left.\sum_{2 k \leq m \leq \lambda / C} \frac{\lambda^{k}}{(m-2 k) ! \lambda^{m}} \partial_{x^{\prime}}^{m-2 k} \partial_{z}^{m} v\right|_{x^{\prime}=z=0} \\
& =\left.\sum_{k \leq m \leq \lambda / C-k} \frac{1}{(m-k) ! \lambda^{m}} \partial_{x^{\prime}}^{m-k} \partial_{z}^{m+k} v\right|_{x^{\prime}=z=0} .
\end{aligned}
$$

Il en résulte que

$$
P_{C}(X)-Q_{C}(X)
$$

$$
=\left.\sum_{k \leq \lambda / C \max (k, \lambda / C-k) \leq m \leq \lambda / C} \frac{X^{k}}{(m-k) ! k !} \frac{1}{\lambda^{m}} \partial_{x^{\prime}}^{m-k} \partial_{z}^{m+k} v\right|_{x^{\prime}=z=0} .
$$

D'après (B.102), on en déduit, avec $\rho=\rho(m)$,

(B.108)

$$
\begin{aligned}
\left|P_{C}(X)-Q_{C}(X)\right| & \leq \sum_{\lambda / 2 C \leq m \leq \lambda / C} \frac{2 m !}{\left(\rho^{2} \lambda\right)^{m}} e^{D \lambda \rho^{3}} \sum_{\lambda / C-m \leq k \leq m} \frac{|X|^{k}}{(m-k) ! k !} e^{\lambda \varphi_{0}} \\
& \leq \sum_{\lambda / 2 C \leq m \leq \lambda / C} \frac{2 m !}{m !\left(\rho^{2} \lambda\right)^{m}} e^{D \lambda \rho^{3}}(1+|X|)^{m} e^{\lambda \varphi_{0}} .
\end{aligned}
$$

On prend alors $\rho(m)=(m / \lambda)^{1 / 3}$, et on obtient

$$
\left|P_{C}(A)-Q_{C}(A)\right| \leq \sum_{\lambda / 2 C \leq m \leq \lambda / C}\left(\frac{4(1+|A|) e^{D}}{C^{1 / 3}}\right)^{m} \leq e^{-\lambda / C+\lambda \varphi_{0}}
$$

dès que $C \geq 64(1+|A|)^{3} e^{3 D+3}$.

Pour écrire le développement de $I$, il reste à expliciter les $k_{n}$ donnés par (B.43). Il suffit pour cela de remarquer que

$$
\int_{0}^{\infty} e^{\lambda x \zeta} \psi(x) \frac{x^{n}}{n !} d x=\left(-\frac{1}{\lambda \zeta}\right)^{n+1}+r_{n}(\zeta)
$$




$$
\begin{aligned}
\left|r_{n}(\zeta)\right| & \leq\left(\frac{3}{\lambda|\operatorname{Re} \zeta|}\right)^{n+1} e^{-2 c t_{0}|\operatorname{Re} \zeta| \lambda}, \\
|\operatorname{Re} \lambda| & \geq|\operatorname{Re} z|+\operatorname{Im} \xi_{0} .
\end{aligned}
$$

Utilisant $\left(\right.$ B.52) avec $\rho=\left(n / \lambda\left(1+\left|\xi_{0}\right|\right)\right)^{1 / 2}$, on obtient:

$$
\left|\sum_{n \leq \lambda / C_{0}} r_{n}(\zeta) \sigma_{n}(0, \xi, \tau, \lambda)\right| \leq D e^{-\lambda / D}
$$

et finalement, passant à la limite si $\nu \rightarrow 0^{+}$, on obtient, en tenant compte de (B.29), (B.42), (B.43), (B.45), (B.74), (B.110),

(B.113)

$$
\begin{aligned}
I= & \sum_{n \leq \lambda / C_{0}} \sum_{m \leq \lambda / C} \frac{1}{2 \pi} \int_{\mathscr{C}\left(l_{1}\right)} d \xi \lambda^{-n-m}\left(\frac{-1}{z+i\left(\xi+a \xi_{0}\right)}\right)^{n+1} \\
& \times\left.\partial_{x}^{n}\left(e^{\lambda\left(i r_{+}(x) \xi_{0}-x^{2} / 2\right)}\left(1-a^{\prime}(x) \frac{\xi}{\xi_{0}}\right)\right)\right|_{x=0} \\
& \times \frac{1}{m !} \partial_{x^{\prime}}^{m}\left[\left.e^{-\lambda\left(x^{\prime 2} / 2+i r_{-}\left(x^{\prime}\right) \xi_{0}\right)} \partial_{z}^{m} F_{-}\left(t-i \tau, x^{\prime}+z-i\left(\xi+b \xi_{0}\right), \lambda\right]\right|_{x^{\prime}=0, z=0}\right. \\
& +R(t, \tau, z, \lambda)
\end{aligned}
$$

où $R$ est négligeable dans $H\left(\varphi_{0}, X_{\kappa}, U\right)$. On notera enfin que, par le même type d'estimations, on peut, dans (B.113), modifier $C_{0}$ par toute constante $\widetilde{C}>C_{0}$.

Preuve de la Proposition 4.3, §4.5. Commençons par démontrer (4.71). En raisonnant comme ci-dessus pour la preuve de la Proposition $4.1 \mathrm{du} \S 4.3$, on est ramené à développer les intégrales

(B.114) $J_{n}(t, \tau, \xi, \lambda)=\sqrt{\frac{\lambda}{2 \pi}} \int_{-\infty}^{+\infty} e^{i \lambda(t-i \tau) \tau^{\prime}-\lambda \tau^{\prime 2} / 2} j_{n}\left(t, \tau, \xi, \tau^{\prime}, \lambda\right) d \tau^{\prime}$,

(B.115) $j_{n}=\int_{0}^{\infty} e^{-i \lambda\left(x^{\prime} \xi+\tau^{\prime} \theta_{a}\left(x^{\prime}\right)\right)} \frac{\partial_{x}^{n} \sigma\left(0, x^{\prime}, \tau, \xi\right)}{n !} p_{2}\left(x^{\prime}, \lambda \tau^{\prime}\right) \psi\left(x^{\prime}\right) d x^{\prime}$,

pour $\xi \in \mathscr{C}_{1}$, avec des estimations en norme $L^{2}$ sur $\mathscr{C}_{1}$.

On écrit alors $\mathscr{C}_{1}=\mathscr{C}_{1}^{+} \cup \mathscr{C}_{1}^{-}$, où $\mathscr{C}_{1}^{-}$est caractérise par

$$
\left|\operatorname{Re} \xi-\frac{1}{\cos \gamma_{+}}\right| \geq 2 D_{1} t_{0}
$$

de sorte que $\operatorname{Im} \xi=-a^{\prime \prime}=-1-4 c t_{0}$ sur $\mathscr{C}_{1}^{-}$. Alors, pour $\xi \in \mathscr{C}_{1}^{-}$,

$$
\left|j_{n}\right| \leq D \lambda^{D}\left(\frac{D_{1}}{a^{\prime}}\right)^{n+1}\left|\xi+\tau^{\prime}\right|^{-1}
$$


donc

(B.118)

$$
\left\|\int_{\left|\tau^{\prime}-1\right| \geq 4 \kappa} e^{i \lambda(t-i \tau) \tau^{\prime}-\lambda \tau^{\prime 2} / 2} j_{n} d \tau^{\prime}\right\|_{L^{2}\left(\mathscr{C}_{1}^{-}\right)} \leq D e^{\lambda \varphi_{0}(t-i \tau)-\lambda / D} .
$$

On écrit alors, pour $\left|\tau^{\prime}-1\right| \leq 4 \kappa$,

$$
j_{n}=\sum_{m \leq \lambda / C_{2}^{0}} \frac{\lambda^{-m-1}}{\left(i\left(\xi+\tau^{\prime}\right)\right)^{m+1}} a_{n, m}+r_{n}
$$

$$
\begin{gathered}
a_{n, m}\left(\tau, \tau^{\prime}, \xi, \lambda\right)=\left.\frac{\partial_{x}^{n}}{n !} \partial_{x^{\prime}}^{m}\left(\sigma\left(0, x^{\prime}, \tau, \xi\right) p_{2}\left(x^{\prime}, \lambda \tau^{\prime}\right) e^{-i \lambda \tilde{\theta}_{a}\left(x^{\prime}\right) \tau^{\prime}}\right)\right|_{x^{\prime}=0}, \\
r_{n}=\sum_{m \leq \lambda / C_{2}^{0}}\left(\int_{0}^{\infty} e^{-i \lambda x^{\prime}\left(\xi+\tau^{\prime}\right)}\left(1-\psi\left(x^{\prime}\right)\right) \frac{x^{\prime m}}{m !} d x^{\prime}\right) a_{n, m}
\end{gathered}
$$

$$
\begin{aligned}
& +\int_{0}^{\infty} e^{-i \lambda x^{\prime}\left(\xi+\tau^{\prime}\right)} \psi\left(x^{\prime}\right) \int_{0}^{x^{\prime}} \frac{\left(x^{\prime}-y^{\prime}\right)^{M}}{M !} \partial_{y^{\prime}}^{M+1} \\
& \quad \times\left(\sigma\left(0, y^{\prime}, \tau, \xi\right) p_{2}\left(y^{\prime}, \lambda \tau^{\prime}\right) e^{-i \lambda \tilde{\theta}_{a}\left(y^{\prime}\right) \tau^{\prime}}\right) d y^{\prime} d x^{\prime}
\end{aligned}
$$

avec $M=\left[\lambda / C_{2}^{0}\right]$.

En intégrant une fois par parties en $x^{\prime}$ et en appliquant dans le terme de rang $m$ l'inégalité de Cauchy avec un rayon égal à $(m / \lambda)^{1 / 2}$, on obtient, si $C_{2}^{0}>\left(D_{1} / a^{\prime}\right)^{2}$

$$
\left\|r_{n}\right\|_{L^{2}\left(\mathscr{C}_{1}^{-}\right)} \leq\left(\frac{D_{1}}{a^{\prime}}\right)^{n+1} \sum_{m \leq \lambda / C_{2}^{0}}\left(\frac{m e^{2}}{\lambda}\right)^{m / 2} e^{-\lambda / D}
$$

$$
\begin{aligned}
& +\left(\frac{D_{1}}{a^{\prime}}\right)^{n+1}\left(\frac{M}{\lambda} e^{2}\right)^{M / 2} \\
\leq & \left(\frac{D_{1}}{a^{\prime}}\right)^{n+1} e^{-\lambda / D}
\end{aligned}
$$

$$
\left|a_{n, m} \lambda^{-m}\right| \leq\left(\frac{D_{1}}{a^{\prime}}\right)^{n+1} e^{-m}
$$

ce qui donne la formule (4.71) pour la partie de l'intégrale correspondant à $\mathscr{C}_{1}^{-}$.

Supposons maintenant $\xi \in \mathscr{C}_{1}^{+}$; alors $\xi$ appartient au domaine

$$
Q=\left\{\zeta \in \mathbb{C},-2<\operatorname{Im} \zeta<a^{\prime},\left|\operatorname{Re} \zeta-\frac{1}{\cos \gamma_{+}}\right|<2 D_{1} t_{0}\right\} .
$$

Pour $\zeta \in Q$, posons

$$
\begin{aligned}
& \tilde{j}_{n}\left(t, \tau, \tau^{\prime}, \xi, \zeta, \lambda\right) \\
& \quad=\int_{0}^{\infty} e^{-i \lambda\left(x^{\prime} \zeta+\theta_{a}\left(x^{\prime}\right) \tau^{\prime}\right)} \frac{\partial_{x}^{n}}{n !} \sigma\left(0, x^{\prime}, \tau, \xi\right) p_{2}\left(x^{\prime}, \lambda \tau^{\prime}\right) \psi\left(x^{\prime}\right) d x^{\prime}
\end{aligned}
$$


(B.126)

$$
\widetilde{J}_{n}(t, \tau, \xi, \zeta, \lambda)=\sqrt{\frac{\lambda}{2 \pi}} \int_{-\infty}^{\infty} e^{+i \lambda(t-i \tau) \tau^{\prime}-\lambda \tau^{\prime 2} / 2} \tilde{j}_{n}\left(t, \tau, \tau^{\prime}, \xi, \zeta, \lambda\right) d \tau^{\prime} .
$$

Nous allons prouver que, pour $\xi \in \mathscr{C}_{1}^{+}, \zeta \in Q$,

$$
\left|\widetilde{J}_{n}(t, \tau, \xi, \zeta, \lambda)\right| \leq D \lambda^{D} e^{\lambda \varphi_{0}(t-i \tau)}\left(\frac{D_{1}}{a^{\prime}}\right)^{n+1} .
$$

Supposons (B.127) prouvé. Alors, définissant $\widetilde{R}_{n}$ par

(B.128) $\tilde{J}_{n}=\sum_{m \leq \lambda / C_{2}^{0}} \sqrt{\frac{\lambda}{2 \pi}} \int_{1-4 \kappa}^{1+4 \kappa} e^{i \lambda(t-i \tau) \tau^{\prime}-\lambda \tau^{\prime 2} / 2} \frac{a_{n, m}\left(\tau, \tau^{\prime}, \xi, \lambda\right)}{\left(i \lambda\left(\zeta+\tau^{\prime}\right)\right)^{m+1}} d \tau^{\prime}+\widetilde{R}_{n}$,

on aura, compte tenu de (B.37) qui est vraie pour tout $\xi \in \mathscr{C}_{1}$,

$$
\left|\widetilde{R}_{n}\right| \leq D \lambda^{D} e^{\lambda \varphi_{0}(t-i \tau)}\left(\frac{D_{1}}{a^{\prime}}\right)^{n+1}
$$

et, par la même preuve que ci-dessus, si $\zeta \in Q$ et $-2<\operatorname{Im} \zeta<-1$,

$$
\left|\widetilde{R}_{n}(t, \tau, \xi, \zeta, \lambda)\right| \leq\left(\frac{D_{1}}{a^{\prime}}\right)^{n+1} e^{\lambda \varphi_{0}(t-i \tau)-\lambda / D}
$$

donc, par le principe du maximum appliqué à la fonction $\zeta \mapsto \widetilde{R}_{n}(\zeta)$ (voir [L2]), on en déduit que, avec une autre constante $D,($ B.130) a encore lieu sur un compact arbitraire de $Q$. En particulier (B.130) a lieu pour $\zeta=\xi \in \mathscr{C}_{1}^{+}$, et (B.128) n'est autre que la formule (4.71) sur $\mathscr{C}_{1}^{+}$.

Passons à la démonstration de (B.127). Tout d'abord, puisque

$$
\left|\tilde{j}_{n}\right| \leq \lambda^{D}\left(\frac{D_{1}}{a^{\prime}}\right)^{n+1} e^{4 \lambda a^{\prime} c t_{0}},
$$

on peut restreindre l'intégrale (B.39) au domaine

$$
\left|\tau^{\prime}-1\right| \leq \varepsilon
$$

dès que

$$
a^{\prime} t_{0} \ll \varepsilon^{2} .
$$

Soit maintenant $u=\operatorname{Re}\left(\zeta+\tau^{\prime}\right)$. Alors, si $\varepsilon$ est assez petit, $u$ est de l'ordre de $1 / \cos \gamma_{+}-1$. On déforme alors l'intégrale (B.125) sur le contour $\Gamma=\Gamma_{1}+\Gamma_{2}$. avec

$$
\begin{aligned}
& \Gamma_{1}: x^{\prime}=-i s u, \quad 0 \leq s \leq \delta \\
& \Gamma_{2}: x^{\prime}=-i \delta g\left(y^{\prime}\right) u+y^{\prime}, \quad 0 \leq y^{\prime},
\end{aligned}
$$

où $g \in C^{\infty}$ est à valeurs dans $[0,1]$, vaut 1 pour $y^{\prime} \leq c t_{0}$, et $g\left(y^{\prime}\right)=0$ pour $y^{\prime} \geq 3 c t_{0}$, et où

$$
0<\delta \ll a^{\prime}
$$


Pour $x^{\prime} \in \Gamma, \zeta \in Q, \xi \in \mathscr{C}_{1}^{+}, \tau^{\prime} \in[1-\varepsilon, 1+\varepsilon]$, et $(t, \tau) \in X_{\kappa}$, on a

$$
\begin{aligned}
\operatorname{Re} \phi: & =\operatorname{Re}\left(i(t-i \tau) \tau^{\prime}-\frac{\tau^{\prime 2}}{2}-i\left(x^{\prime} \zeta+\theta_{a}\left(x^{\prime}\right) \tau^{\prime}\right)\right) \\
& \leq \varphi_{0}(t-i \tau)+\operatorname{Im} x^{\prime} u+\operatorname{Re} x^{\prime} \operatorname{Im}\left(\zeta+\tau^{\prime}\right)+\operatorname{Im}\left(\tilde{\theta}_{a}\left(x^{\prime}\right)\right) \tau^{\prime},
\end{aligned}
$$

soit, compte tenu de (B.135),

$$
\operatorname{Re} \phi \leq \varphi_{0}(t-i \tau)+u \operatorname{Im} x^{\prime}+D a^{\prime} \operatorname{Re} x^{\prime} .
$$

Sur $\Gamma_{1}$, on en déduit $\operatorname{Re} \phi \leq \varphi_{0}(t-i \tau)$, de même que sur $\Gamma_{2}$, si $y^{\prime} \leq c t_{0}$ et si

$$
a^{\prime} t_{0} \ll \delta \text {. }
$$

Il reste à étudier la partie de $\Gamma_{2}$ correspondant à $y^{\prime} \geq c t_{0}$. Pour cela, on déforme l'intégrale en $\tau^{\prime}$ donnant $\widetilde{J}_{n}$, localisée sur $[1-\varepsilon, 1+\varepsilon]$, sur

$$
\operatorname{Re} \tau^{\prime} \in[1-\varepsilon, 1+\varepsilon], \quad \operatorname{Im} \tau^{\prime}=-l
$$

avec $0<l \ll 1$.

Alors la partie réelle de la phase est majorée par

$$
\operatorname{Re} \phi \leq \varphi_{0}(t-i \tau)+D\left(\kappa l+l^{2}\right)-t_{0} \frac{l}{D}+D\left(a^{\prime} t_{0}+t_{0}^{2} l\right)
$$

qui est majorée par $\varphi_{0}(t-i \tau)$ dès que

$$
a^{\prime} \ll l \ll t_{0} .
$$

Enfin, l'erreur commise dans la déformation en $\tau^{\prime}$ est une intégrale dans laquelle $\operatorname{Re} \tau^{\prime}=1 \pm \varepsilon$, donc

$$
\operatorname{Re} \phi \leq \varphi_{0}(t-i \tau)+D\left(\kappa l+l^{2}\right)-\frac{\varepsilon^{2}}{4}+D\left(a^{\prime} t_{0}+t_{0}^{2} l\right)
$$

qui est majorée par $\varphi_{0}(t-i \tau)$ dès que

$$
l \ll \varepsilon, \quad t_{0}^{2} l \ll \varepsilon^{2} .
$$

En reprenant (B.133), (B.135), (B.138), (B.141), et (B.143), on constate que $\delta, l, \varepsilon$ doivent être choisis tels que

(B.144) $\quad \delta \ll a^{\prime} \ll l \ll \min \left(\varepsilon, t_{0}\right), \quad a^{\prime} t_{0} \ll \min \left(\varepsilon^{2}, \delta\right), \quad l t_{0}^{2} \ll \varepsilon^{2}$, ce qui est consistant, puisqu'impliqué par

$$
a^{\prime} t_{0} \ll \delta \ll a^{\prime} \ll l \ll t_{0} \ll \varepsilon \ll 1 .
$$

Passons à (4.73). On a

$$
I_{3}(t, \tau, z, \lambda)=\lim _{\nu \rightarrow 0^{+}} \int_{\xi\left(I_{1}\right)} d \xi e^{-\nu \xi^{2}} k(\tau, z, \xi, \lambda) j_{3}(t, \tau, \xi, \lambda),
$$

où $k$ a déjà été étudié dans la preuve de la Proposition 4.2 du $\$ 4.4$, et où

$$
\begin{aligned}
j_{3}(t, \tau, \xi, \lambda) & =\left(\frac{\lambda}{2 \pi}\right)^{3 / 2} \int_{-\infty}^{\infty} e^{i \lambda(t-i \tau) \tau^{\prime}-\lambda \tau^{\prime 2} / 2} \\
\times & \left(\int_{0}^{\infty} d x^{\prime} \psi\left(x^{\prime}\right) e^{-i \lambda\left(x^{\prime} \xi+h\left(x^{\prime}\right) \xi_{0}+\theta_{h}\left(x^{\prime}\right) \tau^{\prime}\right)} \rho_{3}\left(x^{\prime}, \lambda \tau^{\prime}\right)\right) d \tau^{\prime} .
\end{aligned}
$$


Compte tenu des propriétés du contour $\mathscr{C}\left(l_{1}\right)$ citées dans la preuve de la Proposition $4.2 \mathrm{du} \S 4.4$, on développe $j_{3}$ en décomposant $\mathscr{C}\left(l_{1}\right)=\mathscr{C}\left(l_{1}\right)^{-} \cup \mathscr{C}\left(l_{1}\right)^{+}$, et la démonstration est analogue à la précédente.

\section{Appendice C. Choix de la chaîne D'espaces}

On montre dans cet appendice que l'on peut choisir les ouverts $\omega_{0}$ et $\omega_{1}$ de telle sorte que les opérateurs $s_{n}^{ \pm, \mp}(\tau)$ envoient $H_{0}^{\mp}$ dans $H_{1}^{ \pm}$pour $|\tau-1|<2 \kappa$ (cf. §4.4). Compte tenu de la $\S 4.4$, il suffit de prouver qu'il existe un contour $\Gamma$ du type considéré en (4.54) qui soit contenu dans $i \omega_{0}$, et tel que le contour considéré en (4.56)

$$
\widetilde{\Gamma}: \tilde{\zeta}=\zeta \cos \gamma+\zeta_{0} \sin \gamma
$$

reste à l'extérieur de $i \omega_{1}$. Ici $\xi_{0}=\left(\tau^{2}-\xi^{2}\right)^{1 / 2}$. Le paramètre $\kappa$ pouvant être choisi arbitrairement petit, on peut supposer que $\tau=1$. On choisit alors $\gamma_{0}, \gamma_{1}$ proches de zéro, tels que $0<\gamma_{1}<\gamma_{0}<\gamma$, et on considère les quatre chemins $K_{0}, K_{1}, K, \widetilde{K}=K-\gamma$ dans le plan complexe représenté dans la Figure 16. Alors la transformation $\phi \mapsto i \cos \phi=z$ envoie ces chemins sur les contours représentés dans la Figure 17, p. 420. On choisit ainsi les frontières de $\omega_{0}, \omega_{1}$, en ajustant les paramètres définissant $K_{0}, K_{1}$ par rapport aux constantes du Lemme 4.2. On choisit de même les frontières de $\omega_{\sigma}, \sigma \in[0,1]$, à partir de chemins $K_{\sigma}$ dans le plan des $\phi$ "parallèles" à $K_{0}$ et $K_{1}$, variant linéairement avec $\sigma$. On complète la frontière de $\omega_{\sigma}$ en la raccordant aux demi-droites d'angles polaires $\pi / 2+\delta_{\sigma}$ et $3 \pi / 2-\delta_{\sigma}$. Les propriétés (4.23), (4.24), (4.25), (4.26) sont alors aisées à vérifier.

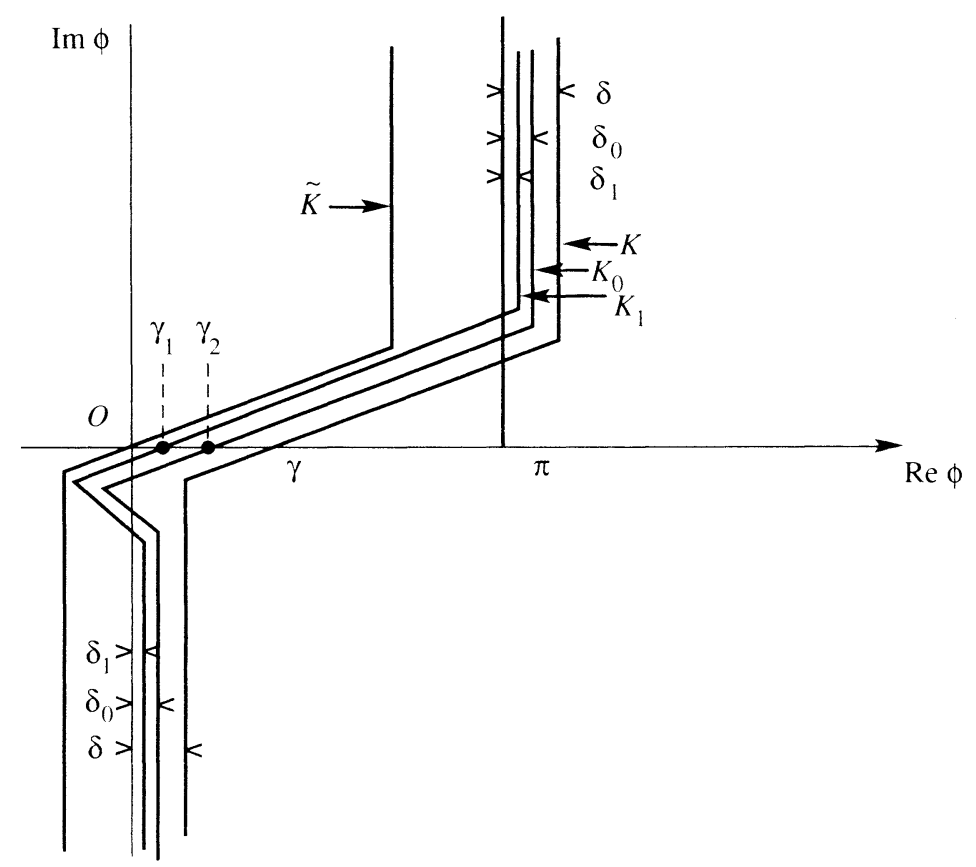

FigURE 16 


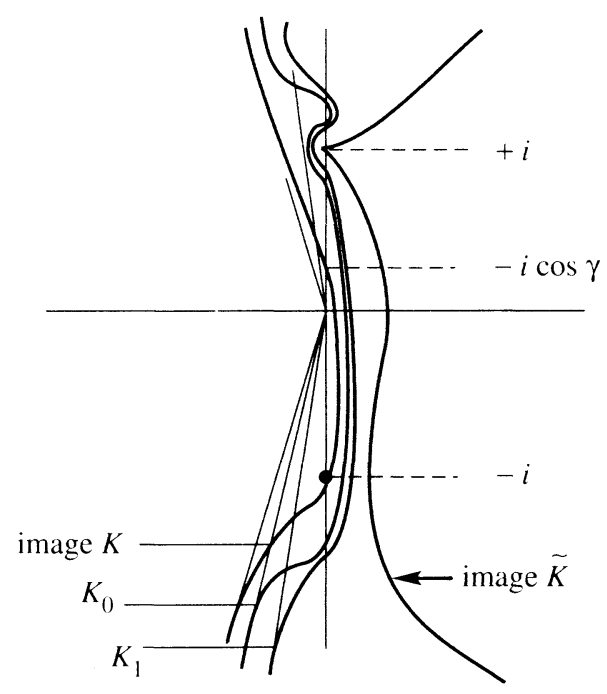

Figure 17

Enfin, si on choisit pour $\Gamma$ l'image par la transformation $\phi \mapsto \zeta=-\cos \phi$ du contour $K$ représenté dans la Figure 1 , on a $\tilde{\zeta}=-\cos (\phi-\gamma)$ donc $\widetilde{\Gamma}$ est l'image par la même transformation du contour $\widetilde{K}=K-\gamma$, et la propriété cherchée devient évidente.

\section{APPENDICE D. RÉSOLUTION D'UN PROBLÈME ELLIPTIQUE DANS UN SECTEUR ANGULAIRE}

Nous démontrons dans cette partie que le problème (5.8) est bien posé. Introduisons préalablement quelques notations. Soit $\alpha \in] 0,2 \pi[$. On désigne par $\Omega$ le secteur angulaire défini en coordonnées polaires $(r, \theta)$ par $0<\theta<\alpha$. On considère l'espace $E$ des distributions $u$ sur $\Omega$ vérifiant en coordonnées polaires

$$
\begin{gathered}
u \in C_{\theta}\left([0, \alpha] ; H_{r}^{1}\left(\mathbb{R}_{+}\right)\right), \quad \frac{1}{r} \partial_{\theta} u \in C_{\theta}\left([0, \alpha] ; L^{2}\left(\mathbb{R}_{+}\right)\right), \\
u(r=0, \theta=0)=u(r=0, \theta=\alpha),
\end{gathered}
$$

$H^{1}$ et $L^{2}$ étant munis de la topologie faible. Si $\chi$ est une fonction $C^{\infty}$ sur $\mathbb{R}^{2}$ qui est constante près de $r=0$ et dans un voisinage de l'infini, alors en écrivant $\Delta=\partial_{r}^{2}+r^{-1} \partial_{r}+r^{-2} \partial_{\theta}$, on constate que $[\Delta, \chi]$ envoie $E$ dans $L^{2}(\Omega)$.

Soit alors $\omega \in \mathbb{C} \backslash \mathbb{R}$.

Théorème D.1. (1) Soit $f \in H^{1}(\partial \Omega)$. Alors le problème

$$
\left(\Delta+\omega^{2}\right) u=0,\left.\quad u\right|_{\partial \Omega}=f
$$

admet dans $E \cap H^{1}(\Omega)$ une unique solution $u$. 
(2) Soit $g \in L^{2}(\partial \Omega)$. Alors le problème

$$
\left(\Delta+\omega^{2}\right) u=0,\left.\quad \partial_{n} u\right|_{\partial \Omega}=g
$$

admet dans $E \cap H^{1}(\Omega)$ une unique solution $u$.

Dans l'assertion (2) du Théorème D.1, $\partial_{n}$ désigne la dérivée normale extérieure à l'ouvert $\Omega$ et coïncide donc avec $r^{-1} \partial_{\theta}$ sur $\{\theta=\alpha\}$ et avec $-r^{-1} \partial_{\theta}$ sur $\{\theta=0\}$. En mettant bout à bout les assertions (1) et (2) ci-dessus, on obtient que le problème $(5.8)$ est bien posé.

On notera que les données au bord dans le Théorème D. 1 sont moins régulières que celles qui sont traditionnellement utilisées dans l'étude des problèmes elliptiques à coins, par exemple dans l'ouvrage de Grisvard [G], auquel nous renvoyons pour le résultat suivant:

Lemme D.1. Soit $h \in L^{2}(\Omega)$.

(1) Le problème

$$
\left(\Delta+\omega^{2}\right) v=h,\left.\quad v\right|_{\partial \Omega}=0
$$

admet une solution et une seule $v \in E \cap H^{1}(\Omega)$.

(2) Le problème

$$
\left(\Delta+\omega^{2}\right) v=h,\left.\quad \partial_{n} v\right|_{\partial \Omega}=0
$$

admet une solution et une seule $v \in E \cap H^{1}(\Omega)$.

Remarque. L'existence d'une solution $H^{1}$ pour les problèmes (1) et (2) du lemme est conséquence immédiate du lemme de Lax-Milgram; à ce sujet, on notera que, puisque $\Omega$ est non borné, l'hypothèse $\omega^{2} \notin \mathbb{R}_{+}$est cruciale.

Passons à la démonstration du Théorème D.1. L'unicité de $u$ découle immédiatement de l'unicité de $v$ dans le lemme. Il suffit bien sûr de construire $u$ lorsque les données sont à support compact près de l'origine et d'autre part lorsque les données sont supportées en dehors de l'origine.

Supposons d'abord que $f$ (resp. $g$ ) est supportée en dehors de l'origine, disons dans $r>1$. Désignons par $P_{1}$ et $P_{2}$ les deux demi-plans dont l'intersection (ou la réunion dans le cas $\alpha>\pi$ ) est $\Omega$. Pour $j=1,2$, on résout dans $P_{j}$

$$
\left(\Delta+\omega^{2}\right) w_{j}=0,\left.\quad w_{j}\right|_{\partial \Omega}=f \quad\left(\text { resp. }\left.\partial_{n} w_{j}\right|_{\partial \Omega}=g\right)
$$

soit $\chi_{j} \in C^{\infty}$ valant 1 sur $\partial P_{j}$ pour $r \geq 1$ et dont le support, proche de celui de $f$, ne rencontre pas $\partial P_{k}$ pour $\{j, k\}=\{1,2\}$. Alors les formules explicites pour la résolution de $\Delta+\omega^{2}$ dans un demi-plan montrent que la fonction $w=\chi_{1} w_{1}+\chi_{2} w_{2}$ appartient à $E(\Omega)$ et satisfait à $\left(\Delta+\omega^{2}\right) w \in L^{2}(\Omega)$. Si $v$ est donnée par le Lemme D.1 avec $h=\left(\Delta+\omega^{2}\right) w$, alors $u=w-v$ est la solution cherchée.

Supposons maintenant que $f$ (resp. $g$ ) soit supportée dans $r<2$. Le lemme suivant indique comment "relever" agréablement les données au bord près de l'origine: 
Lemme D.2. Si $f \in H^{1}(\partial \Omega)$ (resp. $g \in L^{2}(\partial \Omega)$ ), il existe $w$ harmonique dans $\Omega$ telle que

$$
\left.w\right|_{\partial \Omega}=f \quad\left(\text { resp. }\left.\partial_{n} w\right|_{\partial \Omega}=g\right)
$$

et telle que, pour toute fonction $\chi \in C^{\infty}$ à support compact dans le plan, on ait $\chi w \in E$.

Supposant prouvé ce résultat, on choisit $\chi$ à support compact valant 1 pour $r<3$. Alors $h=\left(\Delta+\omega^{2}\right)(\chi w)=[\Delta, \chi] w+\omega^{2} \chi w$ appartient à $L^{2}$, et, si $v$ est donnée par le Lemme D.1, la solution cherchée est $u=\chi w-v$.

Il nous reste à prouver le Lemme D.2. Pour cela, on utilise la transformation de Mellin

$$
M f(z)=\int_{0}^{\infty} f(r) r^{-z} \frac{d r}{r} .
$$

En posant $r=e^{x}$ dans l'intégrale, on constate que $M$ réalise une isométrie (à $2 \pi$ près) de $L^{2}\left(\mathbb{R}_{+}\right)$sur $L^{2}(\operatorname{Re} z=-1 / 2)$, d'inverse donné par

$$
M^{-1} F(r)=\frac{1}{2 i \pi} \int_{\operatorname{Re} z=-1 / 2} F(z) r^{z} d z .
$$

De plus, si $f$ est $L^{2}$ à support borné, alors $M f$ est holomorphe pour $\operatorname{Re} z<$ $-1 / 2$, et, pour tout $a \leq-1 / 2$, on a

$$
\int_{\operatorname{Re} z=a}|M f(z)|^{2}|d z|<\infty .
$$

Enfin, si $f$ est $H^{1}$ à support borné, alors

$$
M f(z)=\frac{1}{z} \int_{0}^{\infty} f^{\prime}(\dot{r}) r^{-z} d r
$$

donc admet un prolongement méromorphe à $\operatorname{Re} z<1 / 2$, le seul pôle étant simple, situé en $z=0$, et de résidu $-f(0)$; on a alors, de plus, pour tout $a \leq 1 / 2$,

$$
\int_{\operatorname{Re} z=a}|z|^{2}|M f(z)|^{2}|d z|<\infty .
$$

Résolvons d'abord le cas du problème de Dirichlet. Pour $f \in H^{1}(\partial \Omega)$, on note $f_{0}=\left.f\right|_{\theta=0}$ et $f_{\alpha}=\left.f\right|_{\theta=\alpha}$. Remarquons que $f_{0}(0)=f_{\alpha}(0)=f(0)$. On pose alors

$$
w(r, \theta)=\frac{1}{2 i \pi} \int_{\operatorname{Re} z=1 / 2} r^{z} W(z, \theta) d z+f(0)
$$

où

$$
W(z, \theta)=\frac{\sin (\alpha-\theta) z}{\sin \alpha z} M f_{0}(z)+\frac{\sin \theta z}{\sin \alpha z} M f_{\alpha}(z) .
$$

On a bien sûr

$$
\Delta w(r, \theta)=\frac{1}{2 i \pi} \int_{\operatorname{Re} z=1 / 2} r^{z-2}\left(z^{2}+\partial_{\theta}^{2}\right) W(z, \theta) d z=0 .
$$


Par ailleurs, puisque $z W$ et $\partial_{\theta} W$ appartiennent à

$$
C\left([0, \alpha] ; L^{2}(\operatorname{Re} z=1 / 2)\right),
$$

on a

$$
\partial_{r} w \in C\left([0, \alpha] ; L^{2}\left(\mathbb{R}_{+}\right)\right), \quad \frac{1}{r} \partial_{\theta} w \in C\left([0, \alpha] ; L^{2}\left(\mathbb{R}_{+}\right)\right)
$$

tandis que $w=r \tilde{w}+f(0)$, où $\tilde{w} \in C\left([0, \alpha] ; L^{2}\left(\mathbb{R}_{+}\right)\right)$. On en déduit que $\chi w \in$ $E$ pour toute fonction $\chi C^{\infty}$ à support borné, sous la réserve que $w(0,0)=$ $w(0, \alpha)$, ce qui sera entraîne par la condition de Dirichlet qu'il nous reste à vérifier. On a

$$
w(r, 0)=\frac{1}{2 i \pi} \int_{\operatorname{Re} z=1 / 2} r^{z} M f_{0}(z) d z+f(0)=f_{0}(r)
$$

d'après la formule de Cauchy et la formule d'inversion pour $M$. On a de même $w(r, \alpha)=f_{\alpha}(r)$, ce qui achève la démonstration dans le cas du problème de Dirichlet. fois

Passons au problème de Neumann. Si $g=\left(g_{0}, g_{\alpha}\right) \in L^{2}(\partial \Omega)$, on pose cette

$$
w(r, \theta)=\frac{1}{2 i \pi} \int_{\operatorname{Re} z=1 / 2} r^{z} W(z, \theta) d z,
$$

où

$$
W(z, \theta)=-\frac{\cos (\alpha-\theta) z}{z \sin \alpha z} M g_{0}(z-1)-\frac{\cos \theta z}{z \sin \alpha z} M g_{\alpha}(z-1) \text {. }
$$

On constate comme ci-dessus que $w$ est harmonique dans $\Omega$ et que

$$
\frac{w}{r}, \partial_{r} w, \frac{1}{r} \partial_{\theta} w \in C\left([0, \alpha] ; L^{2}\left(\mathbb{R}_{+}\right)\right) .
$$

On notera également que $w(0, \theta)=0$. Enfin, $w$ vérifie la condition de Neumann puisque

$$
\frac{1}{r} \partial_{\theta} w(r, 0)=-\frac{1}{2 i \pi} \int_{\operatorname{Re} z=1 / 2} r^{z-1} M g_{0}(z-1) d z=-g_{0}(r)
$$

d'après la formule d'inversion pour $M$. On vérifie de même que $r^{-1} \partial_{\theta} w(r, \alpha)$ $=g_{\alpha}(r)$. Ceci achève la démonstration.

\section{Bibliographie}

[BG] M. S. Baouendi and C. Goulaouic, Remarks on the abstract form of nonlinear CauchyKovalevsky theorems, Comm. Partial Differential Equations 2(1977), 1151-1162.

[CT] J. Cheeger and M. Taylor, Diffraction by conical singularities. 1, Comm. Pure Appl. Math. 35 (1982), 275-331; II, Comm. Pure Appl. Math. 35 (1982), 487-529.

[Ga] H. G. Garnir, Fonction de Green pour Topérateur métaharmonique dans un angle ou un dière, Bull. Soc. Roy. Sci. Liège (1952), 119-140, 207-231, 328-344.

[G] P. Grisvard, Elliptic problems in nonsmooth domains, Monographs and Studies in Mathematics, vol. 24, Pitman, New York, 1985. 
[H] L. Hörmander, The analysis of linear partial differential operators, Vol. 3, Springer, Berlin and New York, 1985.

[KK] M. Kashiwara and T. Kawaï, Microhyperbolic pseudo-differential operators. I, J. Math. Soc. Japan 27 (1975), 359-404.

[Ke] J. B. Keller, Geometrical theory of diffraction, J. Opt. Soc. Amer. 52 (1962), 116-130.

[L1] G. Lebeau, Deuxième microlocalisation à croissance, Séminaire Goulaouic-Meyer-Schwartz, exposé no. 15, 1982-83, Ecole Polytechnique, Palaiseau.

[L2] _ Deuxième microlocalisation sur les sous-variétés isotropes, Lemme III.4, Ann. Inst. Fourier (Grenoble) 35 (1985), 145-216.

[M] H. M. Macdonald, Electric waves, Cambridge University Press, London and New York, 1902, pp. 186-198.

[Mo] F. Molinet, Geometrical theory of diffraction. I, IEEE Antennas and Propagation Society Newsletter, August 1987, pp. 6-17; II, IEEE Antennas and Propagation Society Newsletter, October 1987, pp. 5-16.

[N] L. Nirenberg, An abstract form of the nonlinear Cauchy-Kowalevsky theorem, J. Differential Geom. 6 (1972), 561-576.

[O] L. V. Ovsjannikov, A nonlinear Cauchy problem in a scale of Banach spaces, Dokl. Akad. Nauk SSSR 200 (1971); English transl. in Soviet Math. Dokl. 12 (1971), 1497-1502.

[R] M. Rouleux, Diffraction analytique sur une variété à singularités coniques, Comm. Partial Differential Equations 11 (1986), 947-988.

[Sc] P. Schapira, Propagation at the boundary and reflection of analytic singularities of solutions of linear partial differential equations, Publ. Res. Inst. Math. Sci. 12 Suppl. (1977), 441-453.

[S1] J. Sjöstrand, Propagation of analytic singularities for second order Dirichlet problems. I, Comm. Partial Differential Equations 5 (1980), 41-94.

[S2] __ Singularités analytiques microlocales, Astérisque, no. 95, Société Mathématique de France, Montrouge, 1982.

[S3] _ Analytic singularities and microhyperbolic boundary value problems, Math. Ann. 254 (1980), 211-256.

[U] M. Uchida, Microlocal analysis of diffraction at the corner of an obstacle, Ann. Sci. Ecole Normale Supérieure (4) 25 (1992), 47-75, et Séminaire Equations aux Dérivées Partielles 1989-1990, exposé no. 3, Ecole Polytechnique.

[V] J.-P. Varenne, Diffraction par un angle ou un dièdre, C. R. Acad. Sci. Paris Sér. A 290 (1980), 175-178.

\footnotetext{
ABSTRACT. We study the Dirichlet problem for a wave outside a corner with general analytic boundary.

In particular, we give the asymptotic expansion of the singularity on the diffracted cone.

RESUMÈ. Nous étudions la réflexion d'une onde par un coin à bord analytique, avec condition de Dirichlet.

Nous calculons en particulier l'amplitude diffusée à hautes fréquences sur le cône diffracté.
}

Université de Paris-Sud, Département de Mathématiques, Bâtiment 425, 91405 Orsay, FRANCE

E-mail address, P. Gérard: pgerard@matups.matups.fr

E-mail address, G. Lebeau: lebeau@matups.matups.fr 